\title{
SCALING LIMITS OF LOOP-ERASED RANDOM WALKS AND UNIFORM SPANNING TREES
}

\author{
BY \\ Oded Schramm* \\ Department of Mathematics, The Weizmann Institute of Science, Rehovot 76100, Israel \\ e-mail: schramm@wisdom.weizmann.ac.il \\ http://www.wisdom.weizmann.ac.il/_schramm/
}

ABSTRACT

The uniform spanning tree (UST) and the loop-erased random walk (LERW) are strongly related probabilistic processes. We consider the limits of these models on a fine grid in the plane, as the mesh goes to zero. Although the existence of scaling limits is still unproven, subsequential scaling limits can be defined in various ways, and do exist. We establish some basic a.s. properties of these subsequential scaling limits in the plane. It is proved that any LERW subsequential scaling limit is a simple path, and that the trunk of any UST subsequential scaling limit is a topological tree, which is dense in the plane.

The scaling limits of these processes are conjectured to be conformally invariant in dimension 2. We make a precise statement of the conformal invariance conjecture for the LERW, and show that this conjecture implies an explicit construction of the scaling limit, as follows. Consider the Löwner differential equation

$$
\frac{\partial f}{\partial t}=z \frac{\zeta(t)+z}{\zeta(t)-z} \frac{\partial f}{\partial z}
$$

with boundary values $f(z, 0)=z$, in the range $z \in \mathbf{U}=\{w \in \mathbf{C}:|w|<1\}$, $t \leqslant 0$. We choose $\zeta(t):=\mathrm{B}(-2 t)$, where $\mathrm{B}(t)$ is Brownian motion on $\partial \mathrm{U}$ starting at a random-uniform point in $\partial \mathrm{U}$. Assuming the conformal invariance of the LERW scaling limit in the plane, we prove that the scaling limit of LERW from 0 to $\partial U$ has the same law as that of the path $f(\zeta(t), t)$ (where $f(z, t)$ is extended continuously to $\partial \mathrm{U} \times(-\infty, 0])$. We believe that a variation of this process gives the scaling limit of the boundary of macroscopic critical percolation clusters.

* Research supported by the Sam and Ayala Zacks Professorial Chair. Received May 24, 1999 and in revised form August 1, 1999 
TABLE OF CONTENTS

1. Introduction . . . . . . . . . . . . . . . . 223

2. Some backround and terminology . . . . . . . . . . . . 234

3. No loops . . . . . . . . . . . . . . . . . . 239

4. First steps in the proof of Theorem 1.3 . . . . . . . . . . 245

5. Getting uniform convergence . . . . . . . . . . . . . . . 246

6. Recognizing the Löwner parameter as Brownian motion . . . . 250

7. The winding number of SLE . . . . . . . . . . . . . . . . 252

8. The twisting constant of LERW . . . . . . . . . . . . . . 256

9. The critical value for the SLE . . . . . . . . . . . . . . . 264

10. Properties of UST subsequential scaling limits in two dimensions 265

11. Free and wired trunks and conformal invariance . . . . . . . 280

12. Speculations about the Peano curve scaling limit . . . . . 284

References . . . . . . . . . . . . . . . . . 286

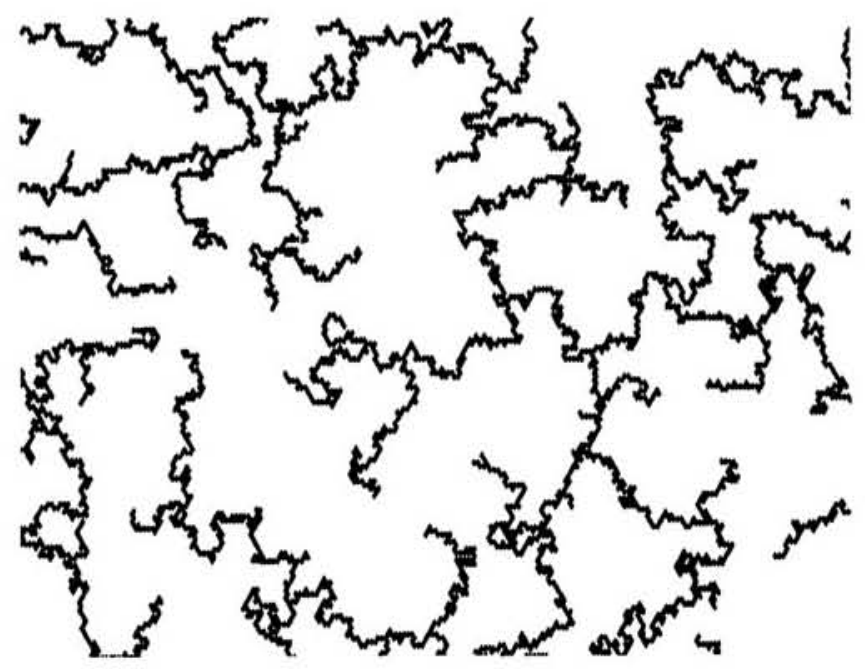

An approximation of the UST scaling limit trunk. 


\section{Introduction}

General REMARKS about SCALING Limits. It is often the case that gridbased probabilistic models should be considered as a mere substitute, or simplification, of a continuous process. There are definite advantages for working in the discrete setting, where unpleasant technicalities can frequently be avoided, simulations are possible, and the setup is easier to comprehend. On the other hand, one is often required to pay some price for the simplification. When we adopt the grid-based world, we sacrifice rotational or conformal symmetries which the continuous model may enjoy, and often have to accept some arbitrariness in the formulation of the model. There are also numerous examples where the continuous process is easier to analyze than the discrete process, and in such situations the continuous may be a useful simplification of the discrete.

Understanding the connections between grid-based models and continuous processes is a project of fundamental importance, and so far has only limited success. As mathematicians, we should not content ourselves with the vague notion that the discrete and continuous models behave "essentially the same", but strive to make the relations concrete and precise.

One reasonable way to define a continuous process is by taking a scaling limit of a grid process. This means making sense of the limit of a sequence of grid processes on finer and finer grids. Recently, Aizenman [Aiz] has proposed a definition for the scaling limit of percolation, and we shall propose a somewhat different definition $[\mathrm{Sch}]$.

Although, in general, the understanding of the connections between grid-based models and continuous models is lacking, there have been some successes. The classical and archetypical example is the relation between simple random walk (SRW) and Brownian motion, which is well studied and quite well understood. See, for example, the discussion of Donsker's Theorem in [Dur91]. We also mention that recently, Tóth and Werner [TW98] have described the scaling limit of a certain self-repelling walk on $\mathbb{Z}$.

The present paper deals with the scaling limits of two very closely related processes, the loop-erased random walk (LERW) and the uniform spanning tree (UST). While these processes are interesting also in dimensions 3 and higher, we restrict attention to two dimensions. In the plane, the scaling limits are conjectured to possess conformal invariance (precise statements appear below), and this can serve as one justification for the special interest in dimension 2. The recent preprint by Aizenman, Burchard, Newman and Wilson [ABNW] discusses scaling limits of random tree processes in two dimensions, including the UST. 
The present work answers some of the questions left open in [ABNW].

The most fundamental task in studying a scaling limit process is to set up a conceptual foundation for the scaling limit. This means answering the following two questions: what kind of object is the scaling limit, and what does it mean to be the scaling limit? For the first question, there's often more than one "right" answer. For example, the scaling limit of (two-sided) simple random walk in $\mathbb{Z}$ is usually defined as a probability measure on $C(\mathbb{R})$, the space of continuous, real-valued functions on $\mathbb{R}$, but it could also be defined as a measure on the space of closed subsets of $\mathbb{R} \times \mathbb{R}$, with an appropriately chosen metric. There's also a "wrong" answer here. It is not a good idea to consider the scaling limit of SRW as a probability measure on $\mathbb{R}^{\mathbb{R}}$, though this might seem at first as more natural.

After the conceptual framework is fixed, the next natural question is the existence of the scaling limit. Unfortunately, we cannot report on any progress here. There is every reason to believe that UST and LERW have scaling limits, but a proof is still lacking. However, in the setup we propose below, the existence of subsequential scaling limits is almost a triviality: for every sequence of positive $\delta_{j}$ tending to 0 , there is a subsequence $\delta_{j_{n}}$ such that the UST and the LERW on the grids $\delta_{j_{n}} \mathbb{Z}^{2}$ do converge to a limit as $n \rightarrow \infty$. Such a limit is called a subsequential scaling limit of the model, and is a probability measure on some space.

All the above discussion concerns foundational issues, which are important. But it is not less important to prove properties of the (subsequential) scaling limit. ${ }^{1}$ In this paper, we prove several almost sure properties of the UST and LERW subsequential scaling limit. We now describe these models and explain the results.

ThE LERW MODEL AND ITS SCALING LIMIT. Consider some set of vertices $K \neq \emptyset$, in a recurrent graph $G$, and a vertex $v_{0}$. The LERW from $v_{0}$ to $K$ in $G$ is obtained by running simple random walk (SRW) from $v_{0}$, erasing loops as they are created, and stopping when $K$ is hit. Here's a more precise description. Let RW be simple random walk starting at $\mathrm{RW}(0)=v_{0}$ and stopped at the first time $\tau$ such that $\operatorname{RW}(\tau) \in K$. Its loop-erasure, $\mathrm{LE}=\mathrm{LE}_{\mathrm{RW}}$, is defined inductively as follows: $\operatorname{LE}(0):=v_{0}$, and $\operatorname{LE}(j+1)=\operatorname{RW}(t+1)$ if $t$ is the last time less than $\tau$ such that $\operatorname{RW}(t)=\operatorname{LE}(j)$. The walk LE stops when it gets to $\operatorname{RW}(\tau) \in K$. Note

1 Sometimes, this can be done without even defining the scaling limit. For example, the Russo-Seymour-Welsh Theorem [Rus78], [SW78] in percolation theory implies properties of any reasonably defined percolation scaling limit. Similarly, Benjamini's paper [Ben] does not explicitly discuss the scaling limit of UST, but has implication to the UST scaling limit. 
that this LERW is a random simple path from $v_{0}$ to $K$.

On a transient graph, it may happen that RW does not hit $K$. However, one can discuss the loop-erasure of the walk continued indefinitely, since it a.s. visits any vertex only finitely many times.

LERW on $\mathbb{Z}^{d}$ was studied extensively by Greg Lawler (see the survey paper [Law] and the references therein), who considered LERW as a simpler substitute for the self-avoiding random walk (see the survey [Sla94]), which is harder to analyze. However, we believe that the LERW model is just as interesting mathematically, because of its strong ties with SRW and UST.

For compactness's sake, in the following we consider the plane $\mathbb{C}=\mathbb{R}^{2}$ as a subset of the two-sphere, $\mathbb{S}^{2}=\mathbb{C} \cup\{\infty\}$, which is the one point compactification of the plane, and work with the spherical metric $d_{s p}$ on $\mathbb{S}^{2}$. Let $D$ be a domain (nonempty open connected set) in the plane $\mathbb{C}=\mathbb{R}^{2}$. We consider a graph $G=G(D, \delta)$, which is an approximation of the domain $D$ in the square grid $\delta \mathbb{Z}^{2}$ of mesh $\delta$. The interior vertices, $\mathrm{V}_{I}(G)$, of $G$ are the vertices of $\delta \mathbb{Z}^{2}$ which are in $D$, and the boundary vertices, $\mathrm{V}_{\partial}(G)$, are the intersections of edges of $\delta \mathbb{Z}^{2}$ with $\partial D$. (The precise definition of $G$ appears in Section 2.) Suppose that each component of $\partial D$ has positive diameter. Let $a \in D$, and let $\mathrm{LE}=\mathrm{LE}_{a, D, \delta}$ be LERW from a vertex $a^{\prime} \in \delta \mathbb{Z}^{2} \cap D$ closest to $a$ to $\mathrm{V}_{\partial}(G)$ in $G$.

To make sense of the concept of the scaling limit of LERW in $D$, we think of LE as a random set in $\bar{D}$. Recall that the Hausdorff distance $d_{\mathcal{H}}(X, Y)$ between two closed nonempty sets $X$ and $Y$ in a compact metric space $Z$ is the least $t \geqslant 0$ such that each point $x \in X$ is within distance $t$ from $Y$ and each point $y \in Y$ is within distance $t$ from $X$; that is,

$$
d_{\mathcal{H}}(X, Y)=\inf \left\{t \geqslant 0: X \subset \bigcup_{x \in X} B(x, t), Y \subset \bigcup_{y \in Y} B(y, t)\right\} .
$$

On the collection $\mathcal{H}(D)$ of closed subsets of $D$, we use the metric $d_{\mathcal{H}(D)}(X, Y):=$ $d_{\mathcal{H}}(X \cup \partial D, Y \cup \partial D)$, and $\mathcal{H}(D)$ is compact with this metric. Then LE $\cap D$ is a random element in $\mathcal{H}(D)$, and its distribution $\mu_{\delta}=\mu_{\delta, D}$ is a probability measure on $\mathcal{H}(D)$. Because the space of Borel probability measures on a compact space is compact in the weak topology, ${ }^{2}$ there is a sequence $\delta_{j} \rightarrow 0$ such that the weak limit $\mu_{0}:=\lim _{j \rightarrow \infty} \mu_{\delta_{j}}$ exists. Such a measure $\mu_{0}$ will be called a subsequential scaling limit measure of LERW from $a$ to $\partial D$. If $\mu_{0}=\lim _{\delta \rightarrow 0} \mu_{\delta}$, then we say that $\mu_{0}$ is the scaling limit measure of LERW from $a$ to $\partial D$.

Similarly, we may consider the scaling limit of LERW between two distinct

2 We review the notion of weak convergence in Section 2 . 
points $a, b \in \mathbb{S}^{2}$, as follows. For $\delta>0$, we take LE to be the loop-erasure of SRW on $\delta \mathbb{Z}^{2}$ starting from a vertex of $\delta \mathbb{Z}^{2}$ within distance $2 \delta$ of $a$ and stopped when it first hits a vertex within distance $2 \delta$ of $b$. Since LE is a.s. compact, its distribution is an element of the Hausdorff space $\mathcal{H}\left(\mathbb{S}^{2}\right)$, and there exists a Borel probability measure on $\mathcal{H}\left(\mathbb{S}^{2}\right)$, which is a subsequential scaling limit measure of the law of LE.

THEOREM 1.1: Let $D$ be a domain in $\mathbb{S}^{2}$ such that each connected component of $\partial D$ has positive diameter, and let $a \in D$. Then every subsequential scaling limit measure of $L E R W$ from a to $\partial D$ is supported on simple paths.

Similarly, if $a, b$ are distinct points in $\mathbb{S}^{2}$, then every subsequential scaling limit of the LERW from $a$ to $b$ in $\delta \mathbb{Z}^{2}$ is supported on simple paths.

Saying that the measure is supported on simple paths means that there's a collection of simple paths whose complement has zero measure.

The CONFORMal INVARIANCE CONJECTURE FOR LERW. Consider two domains $D, D^{\prime} \subset \mathbb{S}^{2}$. Every homeomorphism $f: D \rightarrow D^{\prime}$ induces a homeomorphism $\mathcal{H}(D) \mapsto \mathcal{H}\left(D^{\prime}\right)$. Consequently, if $\mu$ is a probability measure on $\mathcal{H}(D)$, there is an induced probability measure $f_{*} \mu$ on $\mathcal{H}\left(D^{\prime}\right)$.

Conjecture 1.2: Let $D \varsubsetneqq \mathbb{C}$ be a simply connected domain in $\mathbb{C}$, and let $a \in D$. Then the scaling limit of $L E R W$ from a to $\partial D$ exists. Moreover, suppose that $f: D \rightarrow D^{\prime}$ is a conformal homeomorphism onto a domain $D^{\prime} \subset \mathbb{C}$. Then $f_{*} \mu_{a, D}=\mu_{f(a), D^{\prime}}$, where $\mu_{a, D}$ is the scaling limit measure of LERW from a to $\partial D$, and $\mu_{f(a), D^{\prime}}$ is the scaling limit measure of LERW from $f(a)$ to $\partial D^{\prime}$.

Although conformal invariance conjectures have been "floating in the air" in the physics literature for quite some time now, we believe that this precise statement has not yet appeared explicitly. Support for this conjecture comes from simulations which we have performed, and from the work of Rick Kenyon [Ken98a], [Ken98b], [Ken99].

We prove that Conjecture 1.2 implies an explicit description of the LERW scaling limit in terms of solutions of Löwner's differential equation with a Brownian motion parameter. We now give a brief explanation of this.

Let $\mathbb{U}:=\{z \in \mathbb{C}:|z|<1\}$, the unit disk. If $\gamma$ is a compact simple path in $\bar{U}-\{0\}$, such that $\gamma \cap \partial \mathbb{U}$ is an endpoint of $\gamma$, then there is a unique conformal homeomorphism $f_{\gamma}: \mathbb{U} \rightarrow \mathbb{U}-\gamma$ such that $f_{\gamma}(0)=0$ and $f_{\gamma}^{\prime}(0)>0$ (that is, $f_{\gamma}^{\prime}(0)$ is real and positive). Moreover, if $\widehat{\gamma}$ is another such path, and $\widehat{\gamma} \supset \gamma$, then $f_{\gamma}^{\prime}(0)>f_{\hat{\gamma}}^{\prime}(0)$. Now suppose that $\beta$ is a compact simple path in $\overline{\mathbb{U}}$ such that $\partial \mathbb{U} \cap \beta$ is an endpoint of $\beta$ and 0 is the other endpoint of $\beta$, as in Figure 1.1. 
For each point $q \in \beta-\{0\}$, let $\beta_{q}$ be the arc of $\beta$ extending from $q$ to $\partial \mathbb{U}$, and let $h(q):=\log f_{\beta_{q}}^{\prime}(0)$. (If $q$ is the endpoint of $\beta$ on $\partial \mathbb{U}$, then $f_{\beta_{q}}(z)=z$.) It turns out that $h$ is a homeomorphism from $\beta-\{0\}$ onto $(-\infty, 0]$. We let $q(t)$ denote the inverse map $q:(-\infty, 0] \rightarrow \beta$, and set $f(z, t)=f_{t}(z):=f_{\beta_{q(t)}}(z)$. In this setting, Löwner's Slit Mapping Theorem [Löw23] (see also [Pom66]) states that $f_{t}(z)$ is the solution of Löwner's equation

$$
\frac{\partial}{\partial t} f_{t}(z)=z f_{t}^{\prime}(z) \frac{\zeta(t)+z}{\zeta(t)-z}, \quad \forall z \in \mathbb{U}, \forall t \in(-\infty, 0],
$$

where $\zeta:(-\infty, 0] \rightarrow \partial \mathbb{U}$ is some continuous function. In fact, $\zeta(t)$ is defined by the equation

$$
f(\zeta(t), t)=q(t) .
$$

(The left hand side makes sense, since there is a unique continuous extension of $f_{t}$ to $\partial \mathbb{U}$.) Note that $f$ also satisfies

$$
f(z, 0)=z, \quad \forall z \in \mathbb{U} .
$$

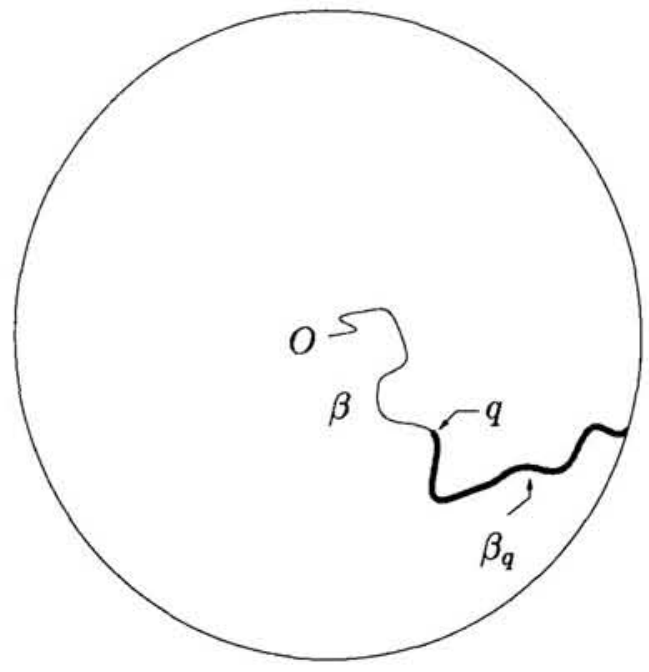

Figure 1.1.

THEOREM 1.3 (The differential equation for the LERW scaling limit): Assume Conjecture 1.2. Let $\mathrm{B}(t), t \geqslant 0$ be Brownian motion on $\partial \mathbb{U}$ starting from a uniform-random point on $\partial \mathbb{U}$. Let $f(z, t)$ be the solution of (1.1) and (1.2), with $\zeta(t):=\mathrm{B}(-2 t)$. Set

$$
\sigma(t)=f(\zeta(t), t), \quad t \leqslant 0 .
$$


Then $\{0\} \cup \sigma((-\infty, 0))$ has the same distribution as the scaling limit of LERW from 0 to $\partial \mathbb{U}$.

The Brownian motion $\mathrm{B}(t)$ in the theorem can be defined as $\mathrm{B}(t):=\exp (i \widehat{\mathrm{B}}(t))$, where $\widehat{B}(t)$ is ordinary Brownian motion on $\mathbb{R}$, starting at a uniform-random point in $[0,2 \pi)$.

Remark 1.4: It is not a priori clear that $\sigma(t)$ is well defined and that $\sigma(t)$ is a simple path. For example, we do not know how to prove that $f_{t}$ extends continuously to $\overline{\mathbb{U}}$ without assuming Conjecture 1.2. The proof of Theorem 1.3 starts by considering the scaling limit of LERW, which is a simple path by Theorem 1.1. It is then shown that the corresponding $\zeta$ has the same distribution as $\mathrm{B}(-2 t)$.

Remark 1.5: Although (1.1) may look like a PDE, it can in fact be presented as an ODE. Set $\Phi(z, t, s):=f_{s}^{-1}\left(f_{t}(z)\right)$ when $t \leqslant s \leqslant 0$. Then

$$
\begin{aligned}
& \Phi(z, t, 0)=f_{t}(z), \\
& \Phi(z, t, t)=z .
\end{aligned}
$$

It is immediate to see that $f_{t}$ satisfies (1.1) iff $\Phi=\Phi(z, t, s)$ satisfies

$$
\frac{\partial \Phi}{\partial s}=-\Phi \frac{\zeta(s)+\Phi}{\zeta(s)-\Phi} .
$$

Therefore, $f_{t}(z)$ can be obtained by solving the ODE (1.5) with $t$ fixed and $s \in[t, 0]$.

Note that $(\zeta+\Phi) /(\zeta-\Phi)$ has positive real part when $\Phi \in \mathbb{U}$ and $\zeta \in \partial \mathbb{U}$. Therefore, (1.5) implies that $|\Phi(z, t, s)|$ is monotone decreasing as a function of $s$. From this it can be deduced that there is a unique solution to the system (1.4) and (1.5) in the interval $s \in[t, 0]$.

Obviously, Theorem 1.3 together with Conjecture 1.2 describe the LERW scaling limit in any simply connected domain $D \varsubsetneqq \mathbb{C}$, since such domains are conformally equivalent to $\mathbb{U}$.

It would be interesting to extract properties of the LERW scaling limit from Theorem 1.3.

At the heart of the proof of Theorem 1.3 lies the following simple combinatorial fact about LERW. Conditioned on a subarc $\beta^{\prime}$ of the LERW $\beta$ from 0 to $\partial D$, which extends from some point $q \in \beta$ to $\partial D$, the distribution of $\beta-\beta^{\prime}$ is the same as that of LERW from 0 to $\partial\left(D-\beta^{\prime}\right)$, conditioned to hit $q$. (See Lemma 4.3.) When we take the scaling limit of this property, and apply the conformal map 
from $\partial\left(D-\beta^{\prime}\right)$ to $\mathbb{U}$, this translates into the Markov property and stationarity of the associated Löwner parameter $\zeta$.

The Uniform SPANNING TREe AND ITS SCALing Limit. Shortly, a definition of the uniform spanning tree (UST) on $\mathbb{Z}^{2}$ will be given. The UST is a statistical-physics model. It lies in the boundary of the two-parameter family of random-cluster measures, which includes Bernoulli percolation and the Ising model [Häg95]. The UST is very interesting mathematically, partly because it is closely related to the theory of resistor networks, potential theory, random walks, LERW, and in dimension 2, also domino tilings. The paper [BLPS98] gives a comprehensive study of uniform spanning trees (and forests), following earlier pioneering work [Ald90], [Bro89], [Pem91], [BP93], [Häg95]. A survey of current UST theory can be found in [Lyo98].

Let $G$ be a connected graph. A forest is a subgraph of $G$ that has no cycles. A tree is a connected forest. A subgraph of $G$ is spanning if it contains $\mathrm{V}(G)$, the set of vertices of $G$. We will be concerned with spanning trees. Since a spanning tree is determined by its edges, we often don't make a distinction between the spanning tree $T$ and its set of edges $E(T)$.

If $G$ is finite, a uniform spanning tree (UST) in $G$ is a random spanning tree $\mathrm{T} \subset G$, selected according to the uniform measure. (That is, $\mathrm{P}\left[\mathrm{T}=T_{1}\right]=$ $\mathrm{P}\left[\mathrm{T}=T_{2}\right]$, whenever $T_{1}$ and $T_{2}$ are spanning trees of $G$.)

It turns out that UST's are very closely related to LERW's. If $a, b \in \mathrm{V}(G)$, then the (unique) path in the UST joining $a$ and $b$ has the same law as the LERW from $a$ to $b$ in $G$. (This, in particular, implies that the LERW from $a$ to $b$ has the same law as the LERW from $b$ to $a$.) Wilson's algorithm [Wil96], which will be described in Section 2, is a very useful method to build the UST by running LERW's.

R. Lyons proposed (see [Pem91]) to extend the notion of UST to infinite graphs. Let $G$ be an infinite connected graph. Consider a nested sequence of connected finite sugraphs $G_{1} \subset G_{2} \subset \cdots \subset G$ such that $G=\bigcup_{j} G_{j}$. For each $j$, the uniform spanning tree measure $\mu_{j}$ on $G_{j}$ may be considered as a measure on $2^{\mathrm{E}(G)}$, the $\sigma$-field of subsets of the edges of $G$, generated by the sets of the form $\{F \subset \mathrm{E}(G): e \in F\}, e \in \mathrm{E}(G)$. Using monotonicity properties, it can be shown that the weak limit $\mu:=\lim _{j \rightarrow \infty} \mu_{j}$ exists, and does not depend on the sequence $\left\{G_{j}\right\}$. It is called the free uniform spanning forest measure (FSF) on $G$. (The reason for the word 'free' is that there's another natural kind of limit, the wired uniform spanning forest (WSF). On $\mathbb{Z}^{d}$, these two measures agree.) R. Pemantle [Pem91] proved that if $d \leqslant 4$, the FSF measure on $\mathbb{Z}^{d}$ is supported on spanning 
trees (that is, if $T$ is random and its law is the FSF measure, then $T$ is a.s. a spanning tree), while if $d \geqslant 5$, the measure is supported on disconnected spanning forests.

Let us now restrict attention to the case $G=\mathbb{Z}^{2}$. Since it is supported on spanning trees, we call the FSF measure on $\mathbb{Z}^{2}$ the uniform spanning tree (UST) on $\mathbb{Z}^{2}$. I. Benjamini [Ben] and R. Kenyon [Ken99] studied asymptotic properties of the UST on a rescaled grid $\delta \mathbb{Z}^{2}$, with $\delta$ small, but did not attempt to define the scaling limit. Aizenman, Burchard, Newman and Wilson [ABNW] defined the scaling limit of UST (and other tree processes) in $\mathbb{Z}^{2}$, and studied some of their properties.

We present a different definition for the scaling limit of the UST. Let $\delta>0$. Again, we think of $\delta \mathbb{Z}^{2}$ as a subset of the sphere $\mathbb{S}^{2}=\mathbb{R}^{2} \cup\{\infty\}$. Let $\widehat{T}_{\delta}$ be the UST on $\delta \mathbb{Z}^{2}$, union with the point at infinity. Then $\hat{\mathrm{T}}_{\delta}$ can be thought of as a random compact subset of $\mathbb{S}^{2}$. However, it is fruitless to consider the weak limit as $\delta \rightarrow 0$ of the law of $\widehat{T}_{\delta}$ as a measure on the Hausdorff space $\mathcal{H}\left(\mathbb{S}^{2}\right)$, since the limit measure is an atomic measure supported on the single point in $\mathcal{H}\left(\mathbb{S}^{2}\right)$, which is all of $\mathbb{S}^{2}$.

Given two points $a, b \in \widehat{\mathrm{T}}_{\delta}$, let $\omega_{a, b}$ be the unique path in $\hat{\mathrm{T}}_{\delta}$ with endpoints $a$ and $b$, allowing for the possibility $\omega_{a, b}=\{a\}$, when $a=b$. (It was proved by $R$. Pemantle that a.s. the UST $T$ in $\mathbb{Z}^{2}$ has a single end; that is, there is a unique infinite ray in $T$ starting at 0 . This implies that indeed $\omega_{a, b}$ exists and is unique, not only for $T$, but also for $T \cup\{\infty\}$.) Let $\mathfrak{T}_{\delta}=\mathfrak{T}_{\delta}\left(\widehat{T}_{\delta}\right)$ be the collection of all triplets, $\left(a, b, \omega_{a, b}\right)$, where $a, b \in \widehat{\mathrm{T}}_{\delta}$. Then $\mathfrak{T}_{\delta}$ is a closed subset of $\mathbb{S}^{2} \times \mathbb{S}^{2} \times \mathcal{H}\left(\mathbb{S}^{2}\right)$, and the law $\mu_{\delta}$ of $\mathfrak{T}_{\delta}$ is a probability measure on the compact space $\mathcal{H}\left(\mathbb{S}^{2} \times \mathbb{S}^{2} \times \mathcal{H}\left(\mathbb{S}^{2}\right)\right)$. By compactness, there is a subsequential weak limit $\mu$ of $\mu_{\delta}$ as $\delta \rightarrow 0$, which is a probability measure on $\mathcal{H}\left(\mathbb{S}^{2} \times \mathbb{S}^{2} \times \mathcal{H}\left(\mathbb{S}^{2}\right)\right)$. We call $\mu$ a subsequential UST scaling limit in $\mathbb{Z}^{2}$. If $\mu:=\lim _{\delta \rightarrow 0} \mu_{\delta}$, as a weak limit, then $\mu$ is the UST scaling limit.

We prove

THEOREM 1.6: Let $\mu$ be a subsequential UST scaling limit in $\mathbb{Z}^{2}$, and let $\mathfrak{T} \in$ $\mathcal{H}\left(\mathbb{S}^{2} \times \mathbb{S}^{2} \times \mathcal{H}\left(\mathbb{S}^{2}\right)\right)$ be a random variable with law $\mu$. Then the following holds a.s.

(i) For every $(a, b) \in \mathbb{S}^{2} \times \mathbb{S}^{2}$, there is some $\omega \in \mathcal{H}\left(\mathbb{S}^{2}\right)$ such that $(a, b, \omega) \in \mathfrak{T}$. For almost every $(a, b) \in \mathbb{S}^{2} \times \mathbb{S}^{2}$, this $\omega$ is unique.

(ii) For every $(a, b, \omega) \in \mathfrak{T}$, if $a \neq b$, then $\omega$ is a simple path, that is, homeomorphic to $[0,1]$. If $a=b$, then $\omega$ is a single point or homeomorphic to a circle. For almost every $a \in \mathbb{S}^{2}$, the only $\omega$ such that $(a, a, \omega) \in \mathfrak{T}$ is $\{a\}$. 
(iii) The trunk,

$$
\text { trunk }:=\bigcup_{(a, b, \omega) \in \mathfrak{T}}(\omega-\{a, b\}),
$$

is a topological tree (in the sense of Definition 10.1), which is dense in $\mathbb{S}^{2}$. (iv) For each $x \in$ trunk, there are at most three connected components of trunk $-\{x\}$.

This theorem basically answers all the topological questions about the UST scaling limit on $\mathbb{Z}^{2}$. It is sharp, in the sense that all the "almost every" clauses cannot be replaced by "every". Benjamini [Ben] proved a result which is closely related to item (iv) of the theorem, and [ABNW] proved (in a different language) that (iv) holds with "three" replaced by some unspecified constant.

The dual of a spanning tree $T \subset \mathbb{Z}^{2}$ is the spanning subgraph of the dual graph $(1 / 2,1 / 2)+\mathbb{Z}^{2}$ containing all edges that do not intersect edges in $T$. It turns out that duality is measure preserving from the UST on $\mathbb{Z}^{2}$ to the UST on the dual grid. The key to the proof of Theorem 1.6 is the statement that the trunk is disjoint from the trunk of the dual UST scaling limit.

Let us stress that Theorem 1.6 is not contingent on Conjecture 1.2. The only contingent theorems proved in this paper are Theorem 1.3, and Theorem 11.3, which says that Conjecture 1.2 implies conformal invariance for the scaling limit of the UST on subdomains of $\mathbb{C}$.

Recent work of R. Kenyon [Ken98a], [Ken98b] proves some conformal invariance results for domino tilings of domains in the plane. There is an explicit correspondence between the UST in $\mathbb{Z}^{2}$ and domino tilings of a finer grid. Based on this correspondence, some properties of the UST can be proved using Kenyon's machinery. For example, Kenyon has shown [Ken98b] that the expected number of edges in a LERW joining two boundary vertices in $\left(\delta \mathbb{Z}^{2}\right) \cap[0,1]^{2}$ (whose distance from each other is bounded from below) grows like $\delta^{-5 / 4}$, as $\delta \rightarrow 0$. He can also show [Ken99] that the weak limit as $\delta \rightarrow 0$ of the distribution of the UST meeting point of three boundary vertices of $D \cap\left(\delta \mathbb{Z}^{2}\right)$ is equivariant with respect to conformal maps. That can be viewed as a partial conformal invariance result for the UST scaling limit. Another example for the applications of Kenyon's work to the UST appears in Section 8. It seems plausible that perhaps soon there would be a proof of Conjecture 1.2.

SLE WITH OTHER PARAMETERS, CRITICAL PERCOlation, AND THE UST Peano CuRve. Let $\kappa \geqslant 0$, and take $\zeta(t):=\mathrm{B}(-\kappa t)$, where $\mathrm{B}$ is as above, Brownian motion on $\partial \mathbb{U}$, started from a uniform random point. Then there is a solution $f(z, t)$ of $(1.1)$ and (1.2), and for each $t \leqslant 0, f_{t}=f(\cdot, z)$ is a conformal 
map from $\mathbb{U}$ into some subdomain $D_{t} \subset \mathbb{U}$. We call the process $\sigma_{t}^{\kappa}:=\mathbb{U}-D_{t}$, $t \leqslant 0$, the stochastic Löwner evolution (SLE) with parameter $\kappa$. It is not always the case that $\sigma_{t}^{\kappa}$ is a simple path. Let $\mathfrak{K}$ be the set of all $\kappa \geqslant 0$ such that for all $t<0$ the set $\sigma_{t}^{\kappa}$ is a.s. a simple path. We show in Section 9 that $\sup \mathfrak{K} \leqslant 4$, and conjecture that $\mathfrak{K}=[0,4]$.

In the past, there has been some work on the question of which Löwner parameters $\zeta$ produce slitted disk mappings ([Kuf47], [Pom66]), but only limited progress has been made. Partly motivated by the present work, Marshall and Rohde [MR] have looked into this problem again, and have shown that when $\zeta$ satisfies a Hölder condition with exponent $1 / 2$ and Hölder(1/2) norm less than some constant, the maps $f_{t}$ are onto slitted disks, and this may fail when $\zeta$ has finite but large Hölder(1/2) norm.

Given some $\kappa>0$, even if $\kappa \notin \mathfrak{K}$, the process $\sigma_{t}^{\kappa}, t \leqslant 0$, is quite interesting. It is a celebrated conjecture that critical Bernoulli percolation on lattices in $\mathbb{R}^{2}$ exhibits conformal invariance in the scaling limit [LPSA94]. Assuming such a conjecture, we plan to prove in a subsequent work that a process similar to SLE describes the scaling limit of the outer boundary of the union of all critical percolation clusters in a domain $D$ which intersect a fixed arc on the boundary of $D$. We also plan to prove that this implies Cardy's [Car92] conjectured formula for the limiting crossing probabilities of critical percolation, and higher order generalizations of this formula.

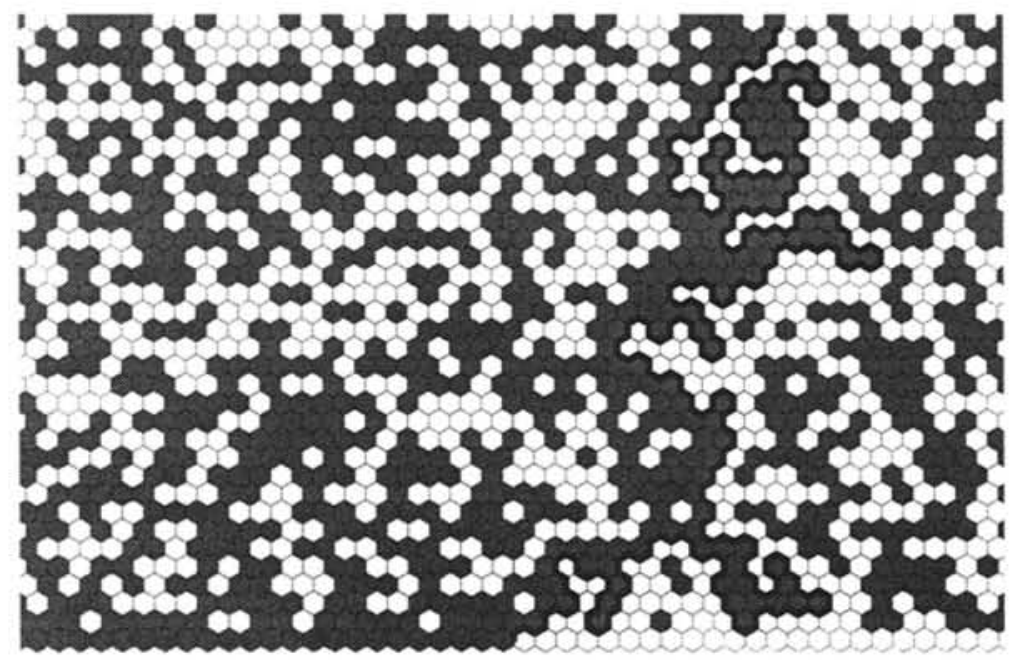

Figure 1.2. The boundary curve for critical percolation with mixed boundary conditions. 
Let us now briefly explain this. In Figure 1.2, each of the hexagons is colored black with probability $1 / 2$, independently, except that the hexagons intersecting the positive real ray are all white, and the hexagons intersecting the negative real ray are all black. Then there is a boundary path $\beta$, passing through 0 and separating the black and the white regions adjacent to 0 . Note that the percolation in the figure is equivalent to Bernoulli $(1 / 2)$ percolation on the triangular grid, which is critical. (See [Gri89] for background on percolation.) The intersection of $\beta$ with the upper half plane, $\mathbb{H}:=\{z \in \mathbb{C}: \operatorname{Im} z>0\}$, which is indicated in the picture, is a random path in $\mathbb{H}$ connecting the boundary points 0 and $\infty$.

A subsequential scaling limit of $\beta \cap \mathbb{H}$ exists, by compactness, and naturally, we believe that the weak limit exists. Let $\gamma$ be the scaling limit curve. The physics wisdom (unproven, perhaps not even precisely formulated, but well supported) is that the scaling limit of the "external boundary" of macroscopic critical percolation clusters in two dimensions has dimension 7/4 and is not a simple path [SD87] (see also [ADA]), and we believe that this is true for $\gamma$.

In a subsequent paper, we plan to prove (by adapting the proof of Theorem 1.3), under the assumption of a conformal invariance conjecture for the scaling limit of critical percolation, that $\gamma$ can be described using a Löwner-like differential equation in the upper half plane with Brownian motion parameter, as follows. Consider the differential equation

$$
\frac{\partial}{\partial t} f_{t}(z)=\frac{-2 f_{t}^{\prime}(z)}{\zeta(t)-z}, \quad \forall z \in \mathbb{H}, \forall t \in(-\infty, 0],
$$

where $\zeta(t)=\mathrm{B}(-\kappa t), \mathrm{B}$ is Brownian motion on $\mathbb{R}$ starting at $\mathrm{B}(0)=0$, and $f_{0}(z)=z$. Then $f_{t}$ is a conformal mapping from $\mathbb{H}$ onto a subdomain of $\mathbb{H}$, which is normalized by the so-called hydrodynamic normalization

$$
\lim _{z \rightarrow \infty} f_{t}(z)-z=0 .
$$

The claim is that for $\kappa=6$, the image of the path $t \mapsto f_{t}(\zeta(t))$ has the same distribution as $\gamma$. From this, one can derive Cardy's [Car92] conjectured formula for the limiting crossing probabilities of critical percolation, as well as some higher order generalizations. This will be done in subsequent work, but basically depends on the ideas appearing in Section 9 below.

A similar representation applies to the scaling limit of the Peano curve which winds around the UST (this curve was discussed in [DD88] and mentioned in [BLPS98]), but with $\kappa=8$. Given a domain $D \subset \mathbb{S}^{2}$, whose boundary is a simple closed path, and given two distinct points $a, b \in \partial D$, there is a naturally 
defined (subsequential) scaling limit of the Peano curve of the UST in $D$, with appropriate boundary conditions, and the scaling limit is an (unparameterized) curve from $a$ to $b$, whose image covers $\bar{D}$. One can show that Conjecture 1.2 implies a conformal invariance property for the scaling limit. Based on this, it should be possible to adapt the proof of Theorem 1.3 to show that Conjecture 1.2 implies a representation of the form (1.6) for this Peano scaling limit when $D=\mathbb{H}, a=0, b=\infty$, and $\kappa=8$. We give a brief overview of this in Section 12, and hope to give a more thorough treatment in a subsequent paper.

The differential equation (1.6) is very similar to Löwner's equation, and the only essential difference is that a normalization at an interior point for the maps $f_{t}$ is replaced by the hydrodynamic normalization (1.7) at a boundary point $(\infty)$. The interior point normalization is natural for the LERW scaling limit, because the LERW is a path from an interior point to the boundary of the domain. The Peano curve and the boundary of percolation clusters, as discussed above, are paths joining two boundary points, and hence the hydrodynamic normalization is more appropriate for them.

Although Löwner's Slit Mapping Theorem mentioned above applies to domains of the form $\mathbb{U}-\alpha$, where $\alpha$ is a simple path in $\mathbb{U}-\{0\}$ with one endpoint in $\partial \mathbb{U}$, Pommerenke [Pom66] has a generalization, which is valid for some paths $\alpha$ which are not simple paths. It is this generalization (or rather, its version in $\mathbb{H}$ with the hydrodynamic normalization) which will substitute Löwner's Slit Mapping Theorem for the treatment of the percolation boundary or Peano curve scaling limits.

The emerging picture is that different values of $\kappa$ in the differential equations (1.1) or (1.6) produce paths which are scaling limits of naturally defined processes, and that these paths can be space-filling, or simple paths, or neither, depending on the parameter $\kappa$.

ACKNowledgement: I wish to express gratitude to Itai Benjamini, Rick Kenyon and David Wilson for inspiring discussions and helpful information. Lemma 3.1 has been obtained jointly with Itai Benjamini. David Aldous, Mladen Bestvina, Brian Bowditch, Steve Evans, Yakar Kannai, Greg Lawler, Russ Lyons, Yuval Peres, Steffen Rohde, Jeff Steif, Benjamin Weiss and Wendelin Werner have provided very helpful advice.

\section{Some background and terminology}

This section will introduce some notations which will be used, and discuss some of the necessary background. We begin with a review of uniform spanning trees 
and forests. The reader may consult [BLPS98] for a comprehensive treatment of that subject.

The DOMINATION PRINCIPLE. Suppose that $H$ and $H^{\prime}$ are two random subsets of some set. We say that $H^{\prime}$ stochastically dominates $H$ if there is a probability measure $\mu$ on pairs $(A, B)$ such that $A$ has the same law as $H, B$ has the same law as $H^{\prime}$, and $\mu\{(A, B): B \supset A\}=1$. Such a $\mu$ is called a monotone coupling of $H$ and $H^{\prime}$.

Let $G$ be a finite connected graph, and let $G_{0}$ be a connected nonempty subgraph. Let $M$ be the set of vertices of $G_{0}$ that are incident with some edge in $\mathrm{E}(G)-\mathrm{E}\left(G_{0}\right)$. (We let $\mathrm{E}(G)$ and $\mathrm{V}(G)$ denote the edges and vertices of $G$, respectively.) Let $G_{0}^{W}$ be the graph obtained from $G$ by identifying all the vertices in $M$ to a single vertex, called the wired vertex. Then $G_{0}^{W}$ is called the wired graph associated to the pair $\left(G_{0}, G\right)$. Let T be the UST on $G$, let $\mathrm{T}_{0}^{F}$ be the UST on $G_{0}$, and let $\mathrm{T}_{0}^{W}$ be the UST on $G_{0}^{W}$. Then $\mathrm{T}_{0}^{F}$ is called the free spanning tree of the pair $\left(G_{0}, G\right)$, and $\mathrm{T}_{0}^{W}$ is the wired spanning tree of the pair $\left(G_{0}, G\right)$. Sometimes, we call $\mathrm{T}_{0}^{F}$ [respectively, $\left.T_{0}^{W}\right]$ the UST on $G_{0}$ with free [respectively, wired] boundary conditions. The domination principle states that $\mathrm{T}_{0}^{F} \cap G_{0}$ stochastically dominates $\mathrm{T} \cap G_{0}$, and that $\mathrm{T} \cap G_{0}$, stochastically dominates $T_{0}^{W} \cap G_{0}$.

Now let $G$ be an infinite connected graph, and let $G_{1} \subset G_{2} \subset \cdots$ be an infinite sequence of finite connected subgraphs satisfying $\bigcup_{j} G_{j}=G$. Let $\mu_{j}^{W}$ be the law of the wired spanning tree of $\left(G_{j}, G\right)$. Based on the domination principle, it is easy to verify that the weak limit $\mu^{W}$ of $\mu_{j}^{W}$ exists, and is a probability measure on spanning forests of $G$. It is called the wired spanning forest of $G$ (WSF).

The domination principle, when appropriatly interpreted, carries over to infinite and to disconnected graphs as well. If $G$ is a disconnected graph, we take the FSF [respectively, WSF] on $G$ to be the spanning forest of $G$ whose intersection with every component of $G$ is the FSF [respectively, WSF] of that component, and with the restriction to the different components being independent. The more general formulation of the domination principle states that when $G_{0}$ is a subgraph of $G$, then $\mathrm{T}_{0}^{F} \cap G_{0}$ stochastically dominates $\mathrm{T} \cap G_{0}$ and $\mathrm{T} \cap G_{0}$ stochastically dominates $\mathrm{T}_{0}^{W} \cap G_{0}$, where $\mathrm{T}, \mathrm{T}_{0}^{F}$ and $\mathrm{T}_{0}^{W}$ are the FSF on $G, G_{0}$ and $G_{0}^{W}$, respectively, and the same statement holds when FSF is replaced by WSF.

On all recurrent connected graphs, the WSF is equal to the FSF, and both are trees. Therefore, on recurrent graphs we shall refer to this measure as the uniform spanning tree (UST).

Grid approximations of Domains. Let $D \subset \mathbb{C}$ be a domain, that is, an 
open, connected set. Given $\delta>0$, we define a graph $G=G(D, \delta)$, which is a discrete approximation of the domain $D$ in the grid $\delta \mathbb{Z}^{2}$, as follows. The interior vertices $\mathrm{V}_{I}(G)=\mathrm{V}_{I}(D, \delta)$ of $G$ are the vertices of $\delta \mathbb{Z}^{2}$ which are in $D$. The boundary vertices $\mathrm{V}_{\partial}(G)=\mathrm{V}_{\partial}(D, \delta)$ are the points of intersection of the edges of the grid $\delta \mathbb{Z}^{2}$ with $\partial D$, the boundary of $D$. The vertices of $G$ are $\mathrm{V}(G)=\mathrm{V}_{\partial}(G) \cup \mathrm{V}_{I}(G)$. If $a, b \in \mathrm{V}(G)$ are distinct, then $[a, b]$ is an edge of $G$ iff there is an edge $e \in \mathrm{E}\left(\delta \mathbb{Z}^{2}\right)$ such that the open segment $\{t a+(1-t) b: t \in(0,1)\}$ is contained in $D \cap e$.

We will often be considering random walks on $G(D, \delta)$ starting at 0 , when $0 \in D$. It will be useful to denote by $\mathrm{V}_{\partial}^{0}$ the set of vertices $v \in \mathrm{V}_{\partial}(D, \delta)$ such that there is a path from 0 in $G(D, \delta)$ whose intersection with $\mathrm{V}_{\partial}$ is $v$.

The wired graph, $G^{W}(D, \delta)$, associated with $D$ is $G(D, \delta)$ with all the vertices $\mathrm{V}_{\partial}(D, \delta)$ collapsed to a single vertex, which we simply denote $\partial D$.

We shall often not distinguish between a graph and its planar embedding, if it has an obvious planar embedding. For example, the UST on $G$ will also be interpreted as a random set in the plane.

WILSON'S ALGORITHM. Let $G$ be a finite graph. Wilson's algorithm [Wil96] for generating a UST in $G$ proceeds as follows. Let $v_{0} \in \mathrm{V}(G)$ be an arbitrary vertex (which we call the root), and set $T_{0}:=\left\{v_{0}\right\}$. Inductively, assume that a tree $T_{j} \subset G$ has been constructed. If $\mathrm{V}\left(T_{j}\right) \neq \mathrm{V}(G)$, choose a vertex $v_{j+1} \in$ $\mathrm{V}(G)-\mathrm{V}\left(T_{j}\right)$, let $W_{j+1}$ be LERW from $v$ to $T_{j}$ in $G$, and set $T_{j+1}:=T_{j} \cup W_{j+1}$. Otherwise $\mathrm{V}\left(T_{j}\right)=\mathrm{V}(G)$, and the algorithm stops and outputs $T_{j}$. It is somewhat surprising, but true, that no matter how the choices of the vertices $v_{j}$ are made, the output of the algorithm is a tree chosen according to the uniform measure.

If $G$ is infinite, connected and recurrent, Wilson's algorithm also "works". When the subtree $T_{j}$ generated by the algorithm includes all the vertices in a certain finite set $K \subset \mathrm{V}(G)$, the subtree of $T_{j}$ spanned by $K$ (that is, the minimal connected subgraph of $T_{j}$ that contains $K$ ) has the same law as the subtree of the UST of $G$ spanned by $K$. (There is also a version of Wilson's algorithm which is useful for generating the WSF of a transient graph, but we shall not need this.)

HARmonic measure estimates. Because of Wilson's algorithm, many questions about the UST can be reduced to questions about simple random walks (SRW's). It is therefore hardly surprising that we often need to obtain a harmonic measure estimate, that is, an estimate on the probability that SRW starting from a vertex $v$ will hit a certain set of vertices $K_{1}$ before hitting another 
set $K_{0}$. As a function of $v$, this probability is harmonic ${ }^{3}$ away from $K_{0} \cup K_{1}$.

Almost all the harmonic measure estimates which we will use are entirely elementary, and follow from the following easy fact. Consider an annulus $A=$ $A(p, r, R)$, with center $p$, inner radius $r$ and outer radius $R>r$. Suppose that $\delta$ is sufficiently small so that there is a path in $\delta \mathbb{Z}^{2} \cap A$ which separates the boundary components of $A$. Let $q \in \delta \mathbb{Z}^{2} \cap A$ be some vertex such that the distance from $q$ to the boundary $\partial A$ is at least $r / c$, where $c>0$ is some constant. Let $X$ be the image of SRW starting from $q$, which is stopped when it first leaves $A$. Then the probability that $X$ contains a path separating the boundary components of $A$ is bounded below by some positive function of $c$. (This can be proved directly using only the Markov property and the invariance of SRW under the automorphisms of $\delta \mathbb{Z}^{2}$.) One consequence of this fact and the Markov property, which we will often use, is as follows.

LEMMA 2.1: Suppose that $K$ is a connected subgraph of $\delta \mathbb{Z}^{2}$ of diameter at least $R$, and $v \in \delta \mathbb{Z}^{2}$. Then the probability that SRW starting from $v$ will exit the ball $B(v, R)$ before hitting $K$ is at most $C_{0}(\operatorname{dist}(v, K) / R)^{C_{1}}$, where $C-0, C_{1}>0$ are absolute constants.

This lemma holds for the spherical as well as Euclidean metric.

LAPLACIAN RANDOM WALK. Although this will not be needed in the paper, we have to mention another interpretation of LERW. Let $G$ be a finite connected graph, let $K \subset \mathrm{V}(G)$ be a set of vertices, and let $a \in \mathrm{V}(G)-K$. The LERW from $a$ to $K$ can also be inductively constructed, as follows. Suppose that the first $n$ vertices $a=\mathrm{LE}(1), \operatorname{LE}(2), \ldots, \operatorname{LE}(n)$ have been determined and $\operatorname{LE}(n) \notin K$. Let $h_{n}: \mathrm{V}(G) \rightarrow[0,1]$ be the function which is 1 on $K, 0$ on $\{\operatorname{LE}(1), \ldots, \operatorname{LE}(n)\}$ and harmonic on $\mathrm{V}(G)-(K \cup\{\operatorname{LE}(1), \ldots, \operatorname{LE}(n)\})$. Then $\operatorname{LE}(n+1)$ is chosen among the neighbors $w$ of $\operatorname{LE}(n)$, with probability proportional to $h_{n}(w)$.

This formulation of the LERW may serve as a heuristic for Conjecture 1.2, since discrete harmonic functions are good approximations for continuous harmonic functions, and continuous harmonic functions in 2D have conformal invariance properties. However, this heuristic is quite weak, since near a non-smooth boundary of a domain, the approximation is not good.

WEAK CONVERGENCE OF MEASURES. We now recall several facts and definitions regarding weak convergence. The reader may consult [EK86, Chap. 3] for proofs and further references. Let $(X, d)$ be a compact metric space, and let $\left\{\mu_{j}\right\}$

3 A function $h$ is harmonic at $v$, if $h(v)$ is the average of the value of $h$ on the neighbors of $v$. 
be a sequence of Borel probability measures on $X$. Let $\mu$ be a Borel probability measure on $X$. Saying that the sequence $\mu_{j}$ converges weakly to $\mu$ means that $\lim _{j} \int f d \mu_{j}=\int f d \mu$ for all continuous $f: X \rightarrow \mathbb{R}$. The Prohorov metric on the space of Borel probability measures on $X$ is defined by

$$
\widehat{d}\left(\mu, \mu^{\prime}\right):=\inf \left\{\epsilon>0: \mu(K) \leqslant \mu^{\prime}\left(N_{\epsilon}(K)\right)+\epsilon \text { for all closed } K \subset X\right\},
$$

where $N_{\epsilon}(K):=\bigcup_{x \in K} B(x, \epsilon)$ is the $\epsilon$-neighborhood of $K$. The space of Borel probability measures on $X$ is compact with respect to the Prohorov metric, and weak convergence is equivalent to convergence in the Prohorov metric.

Let $\mathcal{M}\left(\mu, \mu^{\prime}\right)$ be the collection of all Borel measures $\nu$ on $X \times X$ such that $\nu(A \times X)=\mu(A)$ and $\nu(X \times A)=\mu^{\prime}(A)$ for all measurable $A \subset X$. Such a $\nu$ is called a coupling of $\mu$ and $\mu^{\prime}$. The Prohorov metric satisfies

$$
\widehat{d}\left(\mu, \mu^{\prime}\right)=\inf _{\nu \in \mathcal{M}\left(\mu, \mu^{\prime}\right)} \inf \{\epsilon>0: \nu\{(x, y): d(x, y) \geqslant \epsilon\} \leqslant \epsilon\} .
$$

In other words, $\widehat{d}\left(\mu, \mu^{\prime}\right)<\epsilon$ means that one can find a probability space $(\Omega, \mathbf{P})$ and two $X$ valued random variables $x, y: \Omega \rightarrow X$, such that $\mathbf{P}[d(x, y) \geqslant \epsilon] \leqslant \epsilon$ and such that $x$ has law $\mu$ and $y$ has law $\mu^{\prime}$. This is obtained by taking an appropriate $\mathbf{P}=\nu \in \mathcal{M}\left(\mu, \mu^{\prime}\right), \Omega:=X \times X$, and letting $x$ and $y$ be the projections on the first and second factors, respectively.

CONFORMAL MAPS. We review some elementary facts about conformal mappings, as may be found in [Dur83], for example. Let $D \subset \mathbb{C}$ be some domain. A continuous map $f: D \rightarrow \mathbb{C}$, which is injective and complex-differentiable, is conformal. If $f$ is conformal, then $f^{-1}: f(D) \rightarrow \mathbb{C}$ is also conformal. (Some authors use the word univalent instead of conformal.)

Let $D \varsubsetneqq \mathbb{C}$ be simply connected. Then Riemann's Mapping Theorem states that there is a conformal homeomorphism $f=f_{D}$ from $\mathbb{U}$ onto $D$. Suppose also that $0 \in D$; then $f$ can be chosen to satisfy the normalizations $f(0)=0$ and $f^{\prime}(0)>0$, which render $f$ unique. In this case, the number $f^{\prime}(0)$ is called the conformal radius of $D$ (with respect to 0 ). The Schwarz Lemma implies that

$$
f^{\prime}(0) \geqslant \inf \{|z|: z \notin f(\mathbb{U})\},
$$

while on the other hand, the Koebe $1 / 4$ Theorem gives

$$
f^{\prime}(0) \leqslant 4 \inf \{|z|: z \notin f(\mathbb{U})\} .
$$

Hence, up to a factor of 4 , the conformal radius can be determined from the in-radius. 
If $0<a<b<\infty$, then the set of all conformal maps $f: \mathbb{U} \rightarrow \mathbb{C}$ satisfying $f(0)=0$ and $a \leqslant\left|f^{\prime}(0)\right| \leqslant b$ is compact, in the topology of uniform convergence on compact subsets of $\mathbb{U}$. If $f_{j}: \mathbb{U} \rightarrow \mathbb{C}$ are conformal, $f_{j}(0)=0$, and $f_{j} \rightarrow f$ locally uniformly, then the image $f(\mathbb{U})$ can be described in terms of the images $D_{j}:=f_{j}(\mathbb{U})$. Let $D$ be the maximal open connected set containing 0 and contained in $\bigcup_{n=1}^{\infty} \bigcap_{j=n}^{\infty} D_{j}$. If $D \neq \emptyset$, then $f(\mathbb{U})=D$; otherwise, $f(\mathbb{U})=\{0\}$. This is called Carathéodory's Kernel Convergence Theorem.

If $f: \mathbb{U} \rightarrow D$ is a conformal homeomorphism onto $D$, and $\partial D$ is a simple closed curve, then $f$ extends continuously to $\partial \mathbb{U}$. The same is true if $D=\mathbb{U}-\beta$, where $\beta$ is a simple path.

\section{No loops}

In this section, we prove that any LERW subsequential scaling limit is supported on the set of simple paths. That is, we prove Theorem 1.1.

Let $o_{1}$ and $o_{2}$ be distinct points in $\mathbb{R}^{2}$. For each $\delta>0$, let $o_{1}^{*}$ and $o_{2}^{*}$ be vertices of $\delta \mathbb{Z}^{2}$ closest to $o_{1}$ and $o_{2}$, respectively. Note that in $\delta \mathbb{Z}^{2}$ the combinatorial distance between two vertices $v, v^{\prime} \in \delta \mathbb{Z}^{2}$ is $\delta^{-1}\left\|v-v^{\prime}\right\|_{1}$. However, all metric notions we use will refer to the Euclidean or spherical distance. In this section, we will mainly use the Euclidean metric.

Let RW be a random walk on $\delta \mathbb{Z}^{2}$ starting at $o_{2}^{*}$ and stopped when $o_{1}^{*}$ is reached for the first time. Let $\omega=\omega_{\delta}$ denote the loop-erasure of RW, and let $\mathbf{P}_{\delta}$ denote the law of $\omega_{\delta}$. The following lemma will show that the diameter of $\omega_{\delta}$ is "tight".

LEMMA 3.1:

$$
\mathbf{P}_{\delta}\left[\operatorname{diam} \omega>s \operatorname{dist}\left(o_{1}^{*}, o_{2}^{*}\right)\right] \leqslant C_{0} s^{-C_{1}},
$$

where $C_{0}, C_{1}>0$ are absolute constants.

The proof is based on Wilson's algorithm and an elementary harmonic measure estimate. A more precise estimate can be obtained by using the discrete Beurling Projection Theorem (see [Kes87] or [Law93]).

Proof: Set $r:=\operatorname{dist}\left(o_{1}^{*}, o_{2}^{*}\right), R:=s \operatorname{dist}\left(o_{1}^{*}, o_{2}^{*}\right) / 4$, and let $z \in \delta \mathbb{Z}^{2}$ be some vertex such that $\operatorname{dist}\left(o_{1}^{*}, z\right) \geqslant 10 R$. For $a, b \in \delta \mathbb{Z}^{2}$, let $\omega_{a, b}$ denote the path in the UST of $\delta \mathbb{Z}^{2}$ joining $a$ and $b$. Let $m$ be the meeting point of $o_{1}^{*}, o_{2}^{*}, z$ in the UST, that is, the vertex in $\omega_{o_{\mathbf{1}}^{*}, o_{2}^{*}} \cap \omega_{o_{\mathbf{1}}^{*}, z} \cap \omega_{o_{2}^{*}, z}$. By Wilson's algorithm, the distribution of $\omega$ is identical with the distribution of $\omega_{o_{1}^{*}, m} \cup \omega_{o_{2}^{*}, m}$.

We now estimate $\mathbf{P}\left[\operatorname{diam}\left(\omega_{o_{1}^{*}, m}\right)>s \operatorname{dist}\left(o_{1}^{*}, o_{2}^{*}\right)\right]$. Using Wilson's algorithm, we may generate $\omega_{o_{1}^{*}, m}$ by letting $\omega_{o_{\dot{2}}, z}$ be LERW from $o_{2}^{*}$ to $z$, and letting $\omega_{o_{i}^{*}, m}$ be LERW from $o_{1}^{*}$ to $\omega_{o_{2}^{*}, z}$. Condition on $\omega_{o_{2}^{*}, z}$. By Lemma 2.1, the 
probability that SRW starting at $o_{1}^{*}$ will exit $B\left(o_{1}^{*}, R\right)$ before hitting $\omega_{o_{2}^{*}, z}$ is at most $C_{2}(r / R)^{C_{3}}$, for some constants $C_{2}, C_{3}>0$. Therefore,

$$
\mathrm{P}\left[\operatorname{diam} \omega_{o_{i}^{*}, m}>2 R\right] \leqslant C_{2}(r / R)^{C_{3}} .
$$

Moreover, the same estimate holds for $\operatorname{diam} \omega_{o_{2}^{*}, m}$. Since $\omega_{o_{1}^{*}, o_{2}^{*}}=\omega_{o_{1}^{*}, m} \cup \omega_{o_{2}^{*}, m}$, we get $\mathbf{P}\left[\operatorname{diam} \omega_{o_{i}^{*}, o_{2}^{*}}>4 R\right] \leqslant 2 C_{2}(r / R)^{C_{3}}$. This completes the proof of the lemma.

Remark 3.2: The proof of the lemma can be easily adapted to show that if $a, b \in \delta \mathbb{Z}^{2}$ and $K \subset \delta \mathbb{Z}^{2}$, then the probability that LERW from $a$ to $K$ will intersect $B(b, r)$ is at most $C_{4}(r / \operatorname{dist}(a, b))^{C_{5}}$, provided $r \geqslant \delta$, where $C_{4}, C_{5}>0$ are absolute constants.

Definition 3.3: Let $z_{0} \in \mathbb{R}^{2}, r, \epsilon>0$. An $\left(z_{0}, r, \epsilon\right)$-quasi-loop in a path $\omega$ is a pair $a, b \in \omega$ with $a, b \in B\left(z_{0}, r\right)$, dist $(a, b) \leqslant \epsilon$, such that the subarc of $\omega$ with endpoints $a, b$ is not contained in $B\left(z_{0}, 2 r\right)$. Let $\mathcal{A}\left(z_{0}, r, \epsilon\right)$ denote the set of simple paths in $\mathbb{R}^{2}$ that have a $\left(z_{0}, r, \epsilon\right)$-quasi-loop.

Lemmia 3.4: Let $c$ be the distance from $o_{1}^{*}$ to $o_{2}^{*}$, let $r \in(0, c / 4), \epsilon>0$ and $z_{0} \in \mathbb{R}^{2}$. Then $\lim _{\epsilon \rightarrow 0} \mathbf{P}_{\delta}\left[\mathcal{A}\left(z_{0}, r, \epsilon\right)\right]=0$, uniformly in $\delta$.

Proof: Let $B_{1}=B\left(z_{0}, r\right)$ and $B_{2}=B\left(z_{0}, 2 r\right)$. The distance from $B_{2}$ to at least one of the points $o_{1}^{*}, o_{2}^{*}$ is at least $c / 4$. By symmetry, we assume with no loss of generality that dist $\left(o_{2}^{*}, B_{2}\right) \geqslant c / 4$. Let $\epsilon_{1} \in(0, c / 8)$, and let $q$ be a vertex in $\delta \mathbb{Z}^{2}$ such that $\operatorname{dist}\left(q, o_{2}^{*}\right) \in\left[\epsilon_{1}-\delta, \epsilon_{1}\right]$.

Let $\omega^{q}$ be a LERW from $q$ to $o_{2}^{*}$ in $\delta \mathbb{Z}^{2}$. Let RW be an independent simple random walk from $o_{1}^{*}$. Let $\mathrm{RW}^{\prime}$ be the part of the walk RW until $\omega^{q}$ is first hit. Then, by Wilson's algorithm, $\omega_{\delta}$ has the same distribution as the arc connecting $o_{1}^{*}$ to $o_{2}^{*}$ in $\operatorname{LE}\left(\mathrm{RW}^{\prime}\right) \cup \omega^{q}$.

Let $\mathcal{A}^{\prime}$ be the event that $\operatorname{LE}\left(\mathrm{RW}^{\prime}\right)$ has a $\left(z_{0}, r, \epsilon\right)$-quasi-loop, and let $\mathcal{C}$ be the event that $\omega^{q}$ intersects $B_{2}$. Observe that

$$
\mathcal{A}\left(z_{0}, r, \epsilon\right) \subset \mathcal{A}^{\prime} \cup \mathcal{C} .
$$

Since $\operatorname{dist}\left(q, o_{2}^{*}\right) \leqslant \epsilon_{1}$ and $\operatorname{dist}\left(B_{2}, o_{2}^{*}\right) \geqslant c / 4$, from Lemma 3.1 we get an estimate of the form

$$
\mathbf{P}[\mathcal{C}] \leqslant C_{6}\left(\epsilon_{1} / c\right)^{C_{7}} .
$$

We now find an upper bound for $\mathbf{P}\left[\mathcal{A}^{\prime}-\mathcal{C}\right]$. Let $s_{1}$ be the first time $s \geqslant 0$ such that $\operatorname{RW}(s) \in B_{1}$. Let $t_{1}$ be the first time $t \geqslant s_{1}$ such that $\operatorname{RW}\left(t_{1}\right) \notin B_{2}$. 
Inductively, define $s_{j}$ to be the first time $s \geqslant t_{j-1}$ such that $\mathrm{RW}(s) \in B_{1}$ and $t_{j}$ to be the first time $t \geqslant s_{j}$ such that $\mathrm{RW}(t) \notin B_{2}$. Let $\tau$ be the first time $t \geqslant 0$ such that $\mathrm{RW}(t) \in \omega^{q}$. Finally, for each $s \geqslant 0$ let $\mathrm{RW}^{s}$ be the restriction of RW to the interval $t \in[0, s]$.

For each $j=1,2, \ldots$, we consider several events depending on $\mathrm{RW}^{t_{j}}$ and $\omega^{q}$. Let $\mathcal{Y}_{j}$ be the event that $\operatorname{LE}\left(\mathrm{RW}^{t_{j}}\right)$ has a $\left(z_{0}, r, \epsilon\right)$-quasi-loop. Let $\mathcal{T}_{j}$ be the event that $\tau \geqslant t_{j}$. Observe that $\operatorname{LE}\left(\mathrm{RW}^{t}\right) \cap B_{1} \subset \operatorname{LE}\left(\mathrm{RW}^{t_{j}}\right)$ if $t \in\left[t_{j}, s_{j+1}\right)$. It follows that $\mathcal{A}^{\prime}-\mathcal{C} \subset \mathcal{Y}_{j^{\prime}}$ if $j^{\prime}$ is the largest $j$ with $t_{j} \leqslant \tau$. Therefore,

$$
\mathcal{A}^{\prime}-\mathcal{C} \subset \bigcup_{j=1}^{\infty}\left(\mathcal{Y}_{j} \cap \mathcal{T}_{j}\right) \text {. }
$$

Since $\mathcal{T}_{j} \supset \mathcal{T}_{j+1}$ for each $j$, this implies

$$
\mathcal{A}^{\prime}-\mathcal{C} \subset \mathcal{T}_{m+1} \cup \bigcup_{j=1}^{m} \mathcal{Y}_{j}
$$

for every $m$.

We first estimate $\mathbf{P}\left[\mathcal{T}_{j+1} \mid \mathrm{RW}{ }^{t_{j}}, \omega^{q}\right]$. Conditioned on any $\omega^{q}$, the probability that a SRW starting at any vertex outside of $B_{2}$ will hit $\omega^{q}$ before hitting $B_{1}$ is at least

$$
C_{8}\left(\epsilon_{1}\right)^{C_{9}}
$$

where $C_{8}>0$ depends only on $c$ and $r$, and $C_{9}>0$ is an absolute constant. This is based on the fact that $\omega^{q}$ is connected, contains $o_{2}^{*}$, and has diameter at least $\epsilon_{1}-\delta$. Applying this to the walk RW from time $t_{j}$ on, we therefore get

$$
\mathbf{P}\left[\mathcal{T}_{j+1} \mid \mathrm{RW}^{t_{j}}, \omega^{q}\right] \leqslant 1-C_{8}\left(\epsilon_{1}\right)^{C_{9}} .
$$

By induction, we therefore find that

$$
\mathbf{P}\left[\mathcal{T}_{m} \mid \omega^{q}\right] \leqslant\left(1-C_{8}\left(\epsilon_{1}\right)^{C) 9}\right)^{m-1} .
$$

We now estimate $\mathbf{P}\left[\mathcal{Y}_{j+1} \mid \neg \mathcal{Y}_{j}, \mathrm{RW}^{t_{j}}\right]$. Let $Q_{j}$ be the set of components of $\operatorname{LE}\left(\mathrm{RW}^{s_{j+1}}\right) \cap B_{2}$ that do not contain $\mathrm{RW}\left(s_{j+1}\right)$. Observe that for $\mathcal{Y}_{j+1}$ to occur, there must be a $K \in Q_{j}$ such that the random walk RW comes at some time $t \in\left[s_{j+1}, t_{j+1}\right]$ within distance $\epsilon$ of $K \cap B_{1}$ but $\mathrm{RW}(t) \notin K$ for all $t \in$ $\left[s_{j+1}, t_{j+1}\right]$. But if $\mathrm{RW}(t)$ is close to $K, t \in\left[s_{j+1}, t_{j+1}\right]$, then Lemma 2.1 can be applied, to estimate the probability that RW will not hit $K$ before time $t_{j+1}$. That is, conditioned on $\mathrm{RW}^{s_{j+1}}$, for each given $K \in Q_{j}$, the probability that 
$\mathrm{RW}\left(\left[s_{j+1}, t_{j+1}\right]\right)$ gets to within distance $\epsilon$ of $K$ but does not hit $K$ is at most $C_{1} 0(\epsilon / r)^{C_{1} 1}$, where $C_{1} 0, C_{1} 1>0$ are absolute constants. Consequently, we get

$$
\mathbf{P}\left[\mathcal{Y}_{j+1} \mid \neg \mathcal{Y}_{j}, \mathrm{RW}^{t_{j}}\right] \leqslant C_{1} 0\left|Q_{j}\right|(\epsilon / r)^{C_{1} 1}
$$

Observe that $\left|Q_{j}\right|$, the cardinality of $Q_{j}$, is at most $j$. Therefore,

$$
\mathbf{P}\left[\mathcal{Y}_{j+1} \mid \neg \mathcal{Y}_{j}\right] \leqslant C_{1} 0 j(\epsilon / r)^{C_{1} 1} \text {. }
$$

This gives

$$
\begin{aligned}
\mathbf{P}\left[\bigcup_{j=1}^{m} \mathcal{Y}_{j}\right] \leqslant \sum_{j=1}^{m-1} \mathbf{P}\left[\mathcal{Y}_{j+1} \cap \neg \mathcal{Y}_{j}\right] & \leqslant \sum_{j=1}^{m-1} \mathbf{P}\left[\mathcal{Y}_{j+1} \mid \neg \mathcal{Y}_{j}\right] \\
& \leqslant \sum_{j=1}^{m-1} j C_{1} 0(\epsilon / r)^{C_{1} 1} \leqslant C_{1} 2 m^{2}(\epsilon / r)^{C_{1} 1}
\end{aligned}
$$

Combining this with (3.1), (3.2), (3.3) and (3.4), we find that

$$
\mathbf{P}_{\delta}\left[\mathcal{A}\left(z_{0}, r, \epsilon\right)\right] \leqslant C_{6}\left(\epsilon_{1} / c\right)^{C_{7}}+C_{1} 2 m^{2}(\epsilon / r)^{C_{1} 1}+\left(1-C_{8} \epsilon_{1}^{C_{9}}\right)^{m-1} .
$$

The lemma follows by taking $m:=\left\lfloor\epsilon^{-C_{1} 1 / 3}\right\rfloor$ and $\epsilon_{1}:=-1 / \log \epsilon$, say.

TheOREM 3.5: Let $(X, d)$ be a compact metric space, let $o_{1}, o_{2} \in X$, let $f:(0, \infty)$ $\rightarrow(0, \infty)$ be monotone increasing and continuous, and let $\Gamma=\Gamma(f)$ be the set of all compact simple paths $\gamma \subset X$ with endpoints $o_{1}$ and $o_{2}$ which satisfy the following property. Whenever $x, y$ are points in $\gamma$ and $D(x, y)$ is the diameter of the arc of $\gamma$ joining $x$ and $y$ we have

$$
d(x, y) \geqslant f(D(x, y))
$$

Then $\Gamma$ is compact in the Hausdorff metric.

For this we will need Janiszewski's [Jan12] topological characterization of $[0,1]$ (see [New92, IV.5]):

Lemma 3.6 (Topological Characterization of Arcs): Let $K$ be a compact, connected metric space, and let $o_{1}, o_{2} \in K$. Suppose that for every $x \in K-\left\{o_{1}, o_{2}\right\}$ the set $K-\{x\}$ is disconnected. Then $K$ is homeomorphic to $[0,1]$.

Proof of Theorem 3.5: Let $\mathcal{H}=\mathcal{H}(X)$ denote the space of compact nonempty subsets of $X$ with the Hausdorff metric $d_{\mathcal{H}}$. Let $\gamma$ be in the closure of $\Gamma$ in $\mathcal{H}$. 
Then $\gamma$ is connected, compact, and $\gamma \supset\left\{o_{1}, o_{2}\right\}$. We now use Lemma 3.6 to show that $\gamma$ is a simple path. Indeed, suppose that $x \in \gamma-\left\{o_{1}, o_{2}\right\}$.

We show that $o_{1}$ and $o_{2}$ are in distinct components of $\gamma-\{x\}$. Let $\left\{\gamma_{n}\right\}$ be a sequence in $\Gamma$ such that $d_{\mathcal{H}}\left(\gamma_{n}, \gamma\right)<1 / n$, and let $\left\{x_{n}\right\}$ be a sequence with $x_{n} \in \gamma_{n}$ and $d\left(x_{n}, x\right)<1 / n$. For each $n$ let $\gamma_{n}^{o_{1}}$ be the closed arc of $\gamma_{n}$ with endpoints $o_{1}$ and $x_{n}$, and let $\gamma_{n}^{\sigma_{2}}$ be the closed arc of $\gamma_{n}$ with endpoints $x_{n}$ and $o_{2}$. By passing to a subsequence, if necessary, assume with no loss of generality that the Hausdorff limits $\gamma^{o_{1}}=\lim \gamma_{n}^{o_{1}}$ and $\gamma^{o_{2}}=\lim \gamma_{n}^{o_{2}}$ exist. If $p_{n} \in \gamma_{n}^{o_{1}}, q_{n} \in \gamma_{n}^{o_{2}}$, then $d\left(p_{n}, q_{n}\right) \geqslant f\left(d\left(p_{n}, x_{n}\right)\right)$, since $\gamma_{n} \in \Gamma(f)$. By taking limits we find that if $p \in \gamma^{o_{1}}$ and $q \in \gamma^{o_{2}}$, then $d(p, q) \geqslant f(d(p, x))$. Consequently, $\gamma^{o_{1}}-\{x\}$ and $\gamma^{o_{2}}-\{x\}$ are disjoint. Because $\gamma^{o_{1}} \cup \gamma^{o_{2}}=\gamma$ and $\gamma^{o_{1}}, \gamma^{o_{2}}$ are compact, the set $\gamma-\{x\}$ is not connected. Hence, by Lemma 3.6, $\gamma$ is a simple path.

It remains to prove that $\gamma \in \Gamma(f)$. For any simple path $\beta \subset X$ with endpoints $o_{1}, o_{2}$, let $R(\beta)$ be the set of all $(x, y) \in \beta \times \beta$ such that $x$ belongs to the subarc of $\beta$ with endpoints $o_{1}$ and $y$. With arguments as above, it is not hard to show that $\lim R\left(\gamma_{n}\right)=R(\gamma)$, in the Hausdorff metric on $X \times X$. Since $f$ is continuous, it then easily follows that $\gamma \in \Gamma(f)$. The details are left to the reader. (Actually, one can see that this statement is not essential for the proof of Theorem 1.1. There, we only need the fact that the Hausdorff closure of $\Gamma(f)$ is contained in the set of simple paths.)

Proof of Theorem 1.1: We start with the proof of the second statement, and first assume that $a, b \neq \infty$. Let $o_{1}^{*}$ and $o_{2}^{*}$ be vertices of $\delta \mathbb{Z}^{2}$ closest to $a$ and $b$, respectively. Let $\omega_{\delta}$ be LERW from $o_{1}^{*}$ to $o_{2}^{*}$ in $\delta \mathbb{Z}^{2}$, and let $\omega_{\delta}^{\prime}$ be a path from $a$ to $b$, obtained by taking the line segment joining $a$ to a closest point $a^{\prime}$ on $\omega_{\delta}$, taking the line segment joining $b$ to a closest point $b^{\prime}$ on $\omega_{\delta}$, and taking the path in $\omega_{\delta}$ joining $a^{\prime}$ and $b^{\prime}$. Then the Hausdorff distance from $\omega_{\delta}$ to $\omega_{\delta}^{\prime}$ is less than $2 \delta$. Let $\mathbf{P}_{\delta}^{\prime}$ be the law of $\omega_{\delta}^{\prime}$. Let $\mu$ be some subsequential weak limit of $\mathbf{P}_{\delta}$ as $\delta \rightarrow 0$. Then it is also a subsequential scaling limit of $\mathbf{P}_{\delta}^{\prime}$. Let $m$ be large. By Lemma 3.1 , there is an $R_{m}$ such that with probability at least $1-2^{-m-1}$ we have $\omega_{\delta} \subset B\left(o_{1}, R_{m}\right)$. For each $j \in \mathbb{N}$, let $z_{1}^{j}, \ldots, z_{k_{j}}^{j}$ be a finite set of points in $\mathbb{R}^{2}$ such that the open balls of radius $2^{-j-2}$ about these points cover $\bar{B}\left(o_{1}, R_{m}\right)$. For each $j \in \mathbb{N}$, let $\epsilon_{j}^{m} \in(0,1)$ be sufficiently small so that

$$
\mathbf{P}_{\delta}\left[\mathcal{A}\left(z_{i}^{j}, 2^{-j}, \epsilon_{j}^{m}\right)\right]<2^{-m-2-j} / k_{j},
$$

for all $i=1, \ldots, k_{j}$, and for all $\delta>0$. Such $\epsilon_{j}^{m}$ exist, by Lemma 3.4. Finally, let $f_{m}:(0, \infty) \rightarrow(0, \infty)$ be a continuous monotone increasing function satis- 
fying $f_{m}\left(2^{2-j}\right) \leqslant 2^{-3} \min \left(\epsilon_{j}^{m}, 2^{-j}\right)$ for each $j=1,2, \ldots$ and $\sup _{s>0} f_{m}(s) \leqslant$ $2^{-3} \min \left(\epsilon_{1}^{m}, 1 / 2\right)$.

Let $\mathcal{X}_{m}$ be the space of all compact nonempty subsets of $\bar{B}\left(o_{1}, R_{m}\right)$, and set

$$
\mathcal{Q}_{m}:=\mathcal{X}_{m}-\bigcup_{j=1}^{\infty} \bigcup_{i=1}^{k_{j}} \mathcal{A}\left(z_{i}^{j}, 2^{-j-1}, \epsilon_{j}^{m}\right) .
$$

Note that

$$
\mathbf{P}_{\delta}^{\prime}\left(\neg \mathcal{Q}_{m}\right) \leqslant 2^{-m},
$$

for all $\delta$ and $m$. Also note that if we set $X:=\bar{B}\left(o_{1}, R_{m}\right)$ and $f:=f_{m}$ in Theorem 3.5 , then

$$
\mathcal{Q}_{m} \subset \Gamma_{m}:=\Gamma\left(f_{m}\right)
$$

Indeed, suppose that $\gamma \in \mathcal{X}_{m}$ is a path in $X$ joining $a$ and $b$ and $\gamma \notin \Gamma_{m}$. Then there are $x, y \in \gamma$ and $w$ contained in the arc of $\gamma$ joining $x$ and $y$ such that $\operatorname{dist}(x, y)<2 f(\operatorname{dist}(x, w))$. Since $\sup f \leqslant 2^{-4}$, we have $\operatorname{dist}(x, y)<2^{-3}$. Let $j$ be such that $2^{-j+1} \leqslant \operatorname{dist}(x, w) \leqslant 2^{-j+2}$. Then

$$
\operatorname{dist}(x, y)<2 f(\operatorname{dist}(x, w)) \leqslant 2 f\left(2^{-j+2}\right) \leqslant 2^{-j-2}, \quad \operatorname{dist}(x, y)<2^{-3} \epsilon_{j}^{m} .
$$

Let $i \in\left\{1, \ldots, k_{j}\right\}$ be such that $\operatorname{dist}\left(x, z_{i}^{j}\right) \leqslant 2^{-j-2}$. Then $\operatorname{dist}\left(z_{i}^{j}, w\right) \geqslant 2^{-j}$ and $x, y \in B\left(z_{i}^{j}, 2^{-j-1}\right)$. Consequently, since $d(x, y)<\epsilon_{j}^{m}$, we have

$$
\gamma \in \mathcal{A}\left(z_{i}^{j}, 2^{-j-1}, \epsilon_{j}^{m}\right) .
$$

This proves (3.9).

By (3.8) and (3.9), we get $\mathbf{P}_{\delta}^{\prime}\left(\neg \Gamma_{m}\right) \leqslant 2^{-m}$. Theorem 3.5 tells us that $\Gamma_{m}$ is compact. Therefore, we also have $\mu\left(\neg \Gamma_{m}\right) \leqslant 2^{-m}$, and so

$$
\mu\left(\neg \bigcup_{m} \Gamma_{m}\right)=0 \text {. }
$$

This completes the proof for the case $a, b \neq \infty$, because each element of $\bigcup_{m} \Gamma_{m}$ is a simple path.

The proof when $a$ or $b$ is $\infty$ is similar. One only needs to note that Lemma 3.4 is valid when $z_{0}=\infty$ and the distances are measured in the spherical metric. Indeed, the basic harmonic measure estimate Lemma 2.1 is also valid in the context of the spherical metric. 
The proof of the first statement of Theorem 1.1 is also similar. The details are left to the reader.

Note that the first statement of Theorem 1.1 implies that for almost every subsequential scaling limit path $\gamma$ from $a$ to $\partial D$, the closure of $\gamma \cap D$ intersects $\partial D$ in a single point. This fact is easy to deduce directly, since it is also true for the image of SRW starting near $a$ and stopped when $\partial D$ is hit.

\section{First steps in the proof of Theorem 1.3}

Throughout this section we assume Conjecture 1.2. Let $\sigma$ be random, with the law of the scaling limit of LERW from 0 to $\partial \mathbb{U}$. From Theorem 1.1 we know that $\sigma$ is a.s. a simple path.

Recall that if $D \subset D^{\prime} \subsetneq \mathbb{C}$ are simply connected domains with $0 \in D$, then the conformal radius of $D^{\prime}$ is at least as large as the conformal radius of $D$. This follows from the Schwarz Lemma applied to the map $f_{D^{\prime}}^{-1} \circ f_{D}: \mathbb{U} \rightarrow \mathbb{U}$.

For each $t \in(-\infty, 0]$, let $\sigma_{t}$ be the subarc of $\sigma$ with one endpoint in $\partial \mathbb{U}$ such that the conformal radius of $\mathbb{U}-\sigma_{t}$ is expt. It is clear that $\sigma_{t}$ varies continuously in $t$. Let $f_{t}: \mathbb{U} \rightarrow \mathbb{U}-\sigma_{t}$ be the conformal map satisfying $f_{t}(0)=0$ and $f_{t}^{\prime}(0)=\exp t$. By Löwner's slit mapping theorem [Löw23], there is a unique continuous $\zeta=\zeta_{\sigma}:(-\infty, 0] \rightarrow \partial \mathbb{U}$ such that the differential equation (1.1) holds. Let $\widehat{\zeta}=\widehat{\zeta}_{\sigma}:(-\infty, 0] \rightarrow \mathbb{R}$ be the continuous function satisfying $\zeta(t)=\exp (i \widehat{\zeta}(t))$ and $\widehat{\zeta}(0) \in[0,2 \pi)$. Our goal is to prove

Proposition 4.1: The law of $\widehat{\zeta}$ is stationary, and $\widehat{\zeta}$ has independent increments.

This means that for each $s<0$ the law of the map $t \mapsto \widehat{\zeta}(s+t)$ restricted to $(-\infty, 0]$ is the same as the law of $\widehat{\zeta}$, and that for every $n \in \mathbb{N}$ and $t_{0} \leqslant t_{1} \leqslant$ $\cdots \leqslant t_{n} \leqslant 0$, the increments $\widehat{\zeta}\left(t_{1}\right)-\widehat{\zeta}\left(t_{0}\right), \widehat{\zeta}\left(t_{2}\right)-\widehat{\zeta}\left(t_{1}\right), \ldots, \widehat{\zeta}\left(t_{n}\right)-\widehat{\zeta}\left(t_{n-1}\right)$ are independent. The proof of this proposition, as well as the next, will be completed in later sections.

Note that Conjecture 1.2 implies that the distribution of $\sigma$ is invariant under rotations of $\mathbb{U}$ about 0 . Let $\sigma^{1}$ be random with the law of $\sigma$ conditioned to hit $\partial U$ at 1 . If $\lambda$ denotes the (random) point in $\sigma \cap \partial \mathbb{U}$, then $\sigma^{1}$ has the same law as $\lambda^{-1} \sigma$. It turns out that Proposition 4.1 will follow quite easily from

Proposition 4.2: Assume Conjecture 1.2. Fix some $t<0$. Take $\sigma^{1}$ and $\sigma$ to be independent. As above, let $\sigma_{t}$ be the compact arc of $\sigma$ that has one endpoint on $\partial \mathbb{U}$ and such that the conformal radius of $\mathbb{U}-\sigma_{t}$ is $\exp (t)$. Let $q(t)$ be the endpoint of $\sigma_{t}$ that is in $\mathbb{U}$. Let $\phi$ be the conformal map from $\mathbb{U}$ onto $\mathbb{U}-\sigma_{t}$ satisfying $\phi(0)=0$ and $\phi(1)=q(t)$. Then $\sigma_{t} \cup \phi\left(\sigma^{1}\right)$ has the same law as $\sigma$. 
Now comes an easy lemma about LERW, and Proposition 4.2 will be obtained from this lemma by passing to the scaling limit. The passage to the scaling limit is quite delicate. Recall the definition of the graph $G(D, \delta)$ approximating a domain $D$, from Section 2 .

Lemma 4.3: Let $\delta>0$ and $t<0$ be fixed, and let $D \varsubsetneqq \mathbb{C}$ be a simply connected domain with $0 \in D$. Let $\beta$ be LERW from 0 to $\partial D$ in $G(D, \delta)$. Let $\beta_{t}$ be the compact arc in $\beta$ such that $\beta_{t} \cap \partial D$ is an endpoint of $\beta_{t}$ and such that the conformal radius of $D-\beta_{t}$ is $\exp (t)$. Let $q_{1}(t)$ be the endpoint of $\beta_{t}$ that is not on $\partial \mathbb{U}$. Set $D_{t}=D-\beta_{t}$. Then the law of $\beta-\beta_{t}$ conditioned on $\beta_{t}$ is equal to the law of $L E R W$ from 0 to $\partial D_{t}$, conditioned to hit $q_{1}(t)$.

Proof: There are several different ways to prove this lemma. We prove it using the relation between LERW and the UST. Suppose that $\alpha$ is a path such that $\beta_{t}=\alpha$ has positive probability. We assume for now that the endpoint $q$ of $\alpha$ which is in $D$ lies in the relative interior of an edge $e$ of $\delta \mathbb{Z}^{2}$ (this must be true except for at most a countable possible choices of $t$ ), and set $\tilde{\alpha}:=\alpha-e$. Let $\tilde{q}$ be the endpoint of $\tilde{\alpha}$ in $D$. Let T be the UST on $G^{W}(D, \delta)$, the wired graph of $D$. Then $\beta$ may be taken as the path in T from 0 to $\partial D$. We may generate $T$ using Wilson's algorithm with root $\partial D$, and starting with vertices $v_{1}=\tilde{q}$ and $v_{2}=0$. Conditioning on $\beta_{t}$ being equal to $\alpha$ is the same as conditioning on $\alpha \subset \beta$, which is the same as conditioning on the LERW LE $\mathrm{L}_{1}$ from $v_{1}$ to $\partial D$ to be $\tilde{\alpha}$ and that the LERW from $v_{2}$ to $\mathrm{LE}_{1}$ hits $\tilde{q}$ through the edge $e$. This completes the proof in the case where $q$ is not a vertex of $\delta \mathbb{Z}^{2}$. The case where $q \in \mathrm{V}\left(\delta \mathbb{Z}^{2}\right)$ is treated similarly.

\section{Getting uniform convergence}

The principle goal of this section is to state and prove Proposition 5.5 below. The main point there is that Conjecture 1.2 implies that the weak convergence of loop erased random walk in a domain $D \subset \mathbb{U}$ is uniform in $D$.

The first lemma shows that it is unlikely for a simple random walk to get far away from the boundary of a domain $D$ after getting close to $\partial D$ but before hitting $\partial D$, even if the walk is conditioned to hit $\partial D$ at a certain set of vertices $Q$.

LEMMA 5.1: Let $K$ be a compact connected set in $\overline{\mathbb{U}}$ that contains $\partial \mathbb{U}$ but with $0 \notin K$. Let $F$ be a compact subset of $\mathbb{U}-K$, and let $\epsilon>0$. Then there is a $\delta_{1}>0$ with the following property. Let $K^{\prime}$ be a compact connected set in $\overline{\mathbb{U}}$ with 
$K^{\prime} \supset \partial \mathbb{U}$ and $d_{\mathcal{H}(\mathrm{U})}\left(K, K^{\prime}\right)<\delta_{1} / 5$. Set $D=\mathbb{U}-K^{\prime}$. Let $\delta \in\left(0, \delta_{1} / 5\right)$, and let $Q \subset \mathrm{V}_{\partial}^{0}(D, \delta)$ be nonempty. Let $\mathrm{RW}^{Q}$ be the random walk on $G(D, \delta)$ starting at 0 that stops when it hits $\mathrm{V}_{\partial}(D, \delta)$, conditioned to hit $Q$. Then the probability that $\mathrm{RW}^{Q}$ will reach $F$ after visiting some vertex within distance $\delta_{1}$ of $K$ is less than $\epsilon$.

Proof: We need to recall some basic facts relating the conditioned random walk $\mathrm{RW}^{Q}$ to the unconditioned random walk RW (that stops when hitting $\mathrm{V}_{\partial}(D, \delta)$ ). First recall that $\mathrm{RW}^{Q}$ is a Markov chain. (This is easy to prove directly. See also the discussion of Doob's $h$-transform in [Dur84, §3.1].) Let $v_{0}$ be some vertex, $t_{0} \in \mathbb{N}$, and $W$ a set of vertices. Let $\tau=\tau_{W}$ be the least $t \geqslant t_{0}$ such that $\mathrm{RW}^{Q}(t) \in W$, if such exists, and otherwise set $\tau=\infty$. For $w \in W$ let

$$
a_{W}\left(v_{0}, w\right):=\mathbf{P}\left[\tau<\infty, \operatorname{RW}^{Q}(\tau)=w \mid \mathrm{RW}^{Q}\left(t_{0}\right)=v_{0}\right],
$$

and let $\tilde{a}_{W}\left(v_{0}, w\right)$ be the corresponding quantity for RW. Then

$$
a_{W}\left(v_{0}, w\right)=\frac{\tilde{a}_{W}\left(v_{0}, w\right) h(w)}{h\left(v_{0}\right)},
$$

where $h(v)$ is the probability that RW hits $Q$ when it starts at $v$. This formula is easy to verify.

Let $W_{1}$ be the set of vertices $v$ of $G(D, \delta)$ such that $h(v)<\epsilon h(0) / 5$. By $(5.1)$,

$$
\sum_{w \in W_{1}} a_{W_{1}}(0, w) \leqslant(\epsilon / 5) \sum_{w \in W_{1}} \tilde{a}_{W_{1}}(0, w) \leqslant \epsilon / 5 .
$$

Consequently, the probability that $\mathrm{RW}^{Q}$ visits $W_{1}$ is at most $\epsilon / 5$.

Let $\rho$ be the distance from $F$ to $K$, and assume that $100 \delta_{1}<\rho$. Then an easy discrete Harnack inequality shows that $h(v) / h(0)<C_{0}$ for all vertices $v$ in $F$, where $C_{0}$ is some constant which does not depend on $K^{\prime}$ or $\delta$, but may depend on $F$. There is a first vertex, say $\widehat{v}$, visited by $\mathrm{RW}^{Q}$ such that the distance from $\widehat{v}$ to $K$ is at most $\delta_{1}$. Let $W_{2}$ be the set of vertices of $G(D, \delta)$ in $F$. If $\widehat{v} \notin W_{1}$, then $h(\widehat{v}) \geqslant \epsilon h(0) / 5$ and hence

$$
\sum_{w \in W_{2}} a_{W_{2}}(\widehat{v}, w)=\sum_{w \in W_{2}} \tilde{a}_{W_{2}}(\widehat{v}, w) \frac{h(w)}{h(0)} \frac{h(0)}{h(\widehat{v})} \leqslant 5 C_{0} \epsilon^{-1} \sum_{w \in W_{2}} \tilde{a}_{W_{2}}(\widehat{v}, w) .
$$

But since $K$ is connected, Lemma 2.1 shows that the probability that a simple random walk starting at $\widehat{v}$ will get to distance $\rho / 2$ from $\widehat{v}$ without hitting $V_{\partial}(D, \delta)$ is bounded by $C_{1}\left(\delta_{1} / \rho\right)^{C_{2}}$, where $C_{1}, C_{2}>0$ are absolute constants. By (5.2),

$$
\sum_{w \in W_{2}} a_{W_{2}}(\widehat{v}, w) \leqslant 5 C_{0} \epsilon^{-1} C_{1}\left(\delta_{1} / \rho\right)^{C_{2}} .
$$


Consequently, if $\delta_{1}$ is chosen sufficiently small, the probability that $\mathrm{RW}^{Q}$ starting at $\widehat{v}$ will hit $W_{2}$ is less than $\epsilon / 5$, provided $\widehat{v} \notin W_{1}$. But $\mathbf{P}\left[\widehat{v} \in W_{1}\right] \leqslant \epsilon / 5$, since the probability that $W_{1}$ is visited is at most $\epsilon / 5$. The lemma follows.

In the following, we let $\operatorname{RW}^{D, \delta}$ denote SRW on $G(D, \delta)$ that stops when it hits $\mathrm{V}_{\partial}(D, \delta)$, and let $\mathrm{RW}^{D, \delta, Q}$ denote $\mathrm{RW}^{D, \delta}$ conditioned to hit $Q$, if $Q \subset \mathrm{V}_{\partial}^{0}(D, \delta)$. Suppose that $\nu$ is a probability measure on $\mathrm{V}_{\partial}^{0}(D, \delta)$ and $p$ is random with law $\nu$; then $\mathrm{RW}^{D, \delta, \nu}$ will denote $\mathrm{RW}^{D, \delta}$ conditioned to hit $p$ given $p$. In other words, the law of $\mathrm{RW}^{D, \delta, \nu}$ is the convex combination of the laws of the walks $\mathrm{RW}^{D, \delta,\{p\}}$, with coefficients $\nu(\{p\})$.

Lemma 5.2: Assume Conjecture 1.2. Let $D \subset \mathbb{U}$ be a Jordan domain with $0 \in D$. Let $\phi: D \rightarrow \mathbb{U}$ be the conformal homeomorphism from $D$ to $\mathbb{U}$ satisfying $\phi(0)=0, \phi^{\prime}(0)>0$. Then as $\delta \rightarrow 0$ the law of the pair

$$
\left(\phi\left(\operatorname{LE}\left(\mathrm{RW}^{D, \delta}\right)\right), \partial \mathbb{U} \cap \phi\left(\mathrm{RW}^{D, \delta}\right)\right)
$$

tends weakly to the law of the pair $(\sigma, \partial \mathbb{U} \cap \sigma)$.

This lemma is easily proved using arguments as in the proof of Lemma 5.1, and is therefore left to the reader.

If $X$ and $Y$ are random closed subsets of $\mathbb{U}$, we let $\widehat{d}_{\mathbf{U}}(X, Y)$ denote the Prohorov distance between the law of $X \cup \partial \mathbb{U}$ and the law of $Y \cup \partial \mathbb{U}$ (see Section 2, towards the end), where the metric $d_{\mathcal{H}(\overline{\mathrm{U}})}$ is used on $\mathcal{H}(\overline{\mathbb{U}})$. If $F$ is a subset of $\overline{\mathbb{U}}$, we set $\widehat{d}_{F}(X, Y):=\widehat{d}_{\mathrm{U}}(X \cup \overline{\mathbb{U}-F}, Y \cup \overline{\mathbb{U}-F})$. This is a measure of how much $X$ and $Y$ differ inside $F$.

The next lemma shows that up to a specified accuracy the loop-erased random walk on a domain $D$ can be approximated by the loop-erased random walk on another domain $D^{\prime}$, where $D^{\prime}$ is selected from a finite collection of smooth Jordan domains.

Lemma 5.3: Let $\epsilon>0$. Then there is a $\delta_{0}>0$ and a finite collection of smooth Jordan domains $D_{1}, D_{2}, \ldots, D_{n} \subset \mathbb{U}$ with $0 \in D_{j}$ for all $j$, and with the following property. Let $D \subset \mathbb{U}$ be a simply connected domain with $B(0, \epsilon) \subset D$, let $\delta \in\left(0, \delta_{0}\right)$ and let $Q \subset \mathrm{V}_{\partial}^{0}(D, \delta)$ be nonempty. Let $F_{\epsilon}$ be the component of 0 in the set of points in $D$ that have distance at least $\epsilon$ to $\partial D$. Then there is a $D^{\prime} \in\left\{D_{1}, \ldots, D_{n}\right\}$ and a probability measure $\nu$ on $\mathrm{V}_{\partial}^{0}\left(D^{\prime}, \delta\right)$ such that

$$
\widehat{d}_{F_{\epsilon}}\left(\operatorname{LE}\left(\operatorname{RW}^{D, \delta, Q}\right), \operatorname{LE}\left(\operatorname{RW}^{D^{\prime}, \delta, \nu}\right)\right)<\epsilon .
$$

Moreover, we may require that the Hausdorff distance from $\partial D$ to $\partial D^{\prime}$ is at most $\epsilon$. 
Proof: We first prove the lemma for some specific $D$ and $Q$ as above. By Lemma 5.1 , there is a $\delta_{1}>0$ such that with probability $\geqslant 1-\epsilon / 5$ the walk $\mathrm{RW}^{D, \delta, Q}$ does not reach $F_{\epsilon}$ after exiting $F_{\delta_{1}}$, provided $\delta \in\left(0, \delta_{1} / 5\right)$. Also, there is a $\delta_{1}^{\prime}<\delta_{1}$ such that with probability $\geqslant 1-\epsilon / 5$ this walk does not reach $F_{\delta_{1}}$ after exiting $F_{\delta_{1}^{\prime}}$. Let $D^{\prime} \subset D$ be a smooth Jordan domain with $D^{\prime} \supset F_{\delta_{1}^{\prime}}$, and such that the Hausdorff distance from $\partial D^{\prime}$ to $\partial D$ is less than $\epsilon$. For every $\delta<\delta_{1}^{\prime} / 5$, let $\nu=\nu_{\delta}$ be the hitting measure of $\mathrm{RW}^{D, \delta, Q}$ on $\mathrm{V}_{\partial}^{0}\left(D^{\prime}, \delta\right)$. Observe that we may think of $\mathrm{RW}^{D^{\prime}, \delta, \nu}$ as equal to $\mathrm{RW}^{D, \delta, Q}$ stopped when $V_{\partial}\left(D^{\prime}, \delta\right)$ is hit.

Let $\mathcal{A}_{1}$ be the event that $\mathrm{RW}^{D, \delta, Q}$ does not visit $F_{\epsilon}$ after exiting $F_{\delta_{1}}$, and let $\mathcal{A}_{2}$ be the event that $\mathrm{RW}^{D, \delta, Q}$ does not visit $F_{\delta_{1}}$ after exiting $F_{\delta_{1}^{\prime}}$. Note that on the event $\mathcal{A}_{1} \cap \mathcal{A}_{2}$, after exiting $F_{\delta_{1}^{\prime}}$ the random walk does not visit any vertex $v$ which was already visited prior to the last visit to $F_{\epsilon}$. Consequently, the intersection of $F_{\epsilon}$ with the loop erasure of the walk does not change after the first exit of $F_{\delta_{1}^{\prime}}$. Since we may couple $\mathrm{RW}^{D^{\prime}, \delta, \nu}$ to equal $\mathrm{RW}^{D, \delta, Q}$ stopped on $V_{\partial}\left(D^{\prime}, \delta\right)$, this means that we may obtain a coupling giving $F_{\epsilon} \cap \operatorname{LE}\left(\mathrm{RW}^{D, \delta, Q}\right)=F_{\epsilon} \cap \operatorname{LE}\left(\mathrm{RW}^{D^{\prime}, \delta, \nu}\right)$ on $\mathcal{A}_{1} \cap A_{2}$. Since $\mathrm{P}\left[\mathcal{A}_{1} \cap \mathcal{A}_{2}\right] \geqslant 1-\epsilon / 2$, this proves the lemma for a single $D$. However, the same pair $\left(D^{\prime}, \delta_{1}\right)$ would work for every $D^{\prime \prime}$ with $\partial D^{\prime \prime}$ sufficiently close to $\partial D$ in the Hausdorff metric. Hence, the compactness of the Hausdorff space of compact, connected subsets of $\overline{\mathbb{U}}-B(0, \epsilon)$ completes the proof.

Lemma 5.4: Let $D \subset \mathbb{U}$ be a smooth Jordan domain with $0 \in D$, let $\delta>0$, let $q \in \mathrm{V}_{\partial}^{0}(D, \delta)$, and let $\mathrm{RW}^{q}:=\mathrm{RW}^{D, \delta,\{q\}}$. Then the law of $\mathrm{LE}\left(\mathrm{RW}^{q}\right)$ is uniformly continuous in $q$. That is, for every $\epsilon>0$ there is a $\delta_{1}>0$ such that

$$
\widehat{d}_{D}\left(\operatorname{LE}\left(\operatorname{RW}^{q}\right), \operatorname{LE}\left(\operatorname{RW}^{q^{\prime}}\right)\right)<\epsilon
$$

provided $\delta \in\left(0, \delta_{1}\right)$, and $\left|q-q^{\prime}\right|<\delta_{1}$.

Proof: Let $\delta_{0}>0$ be very small. It is easy to see that when $\left|q-q^{\prime}\right|$ is small we may couple $\mathrm{RW}^{q}$ and $\mathrm{RW}^{q^{\prime}}$ so that with probability at least $1-\epsilon / 5$ they are equal until they both come within distance $\delta_{0}$ of $\partial D$. Hence, the lemma follows by using an argument similar to the one used in the proof of Lemma 5.3.

We now come to the principle goal of this section, proving that Conjecture 1.2 implies that the weak convergence of loop erased random walk in a domain $D \subset \mathbb{U}$ is uniform in $D$.

Proposition 5.5: Assume Conjecture 1.2, and let $\epsilon>0$. Then there is a $\delta_{1}>0$ with the following property. Let $D \subset \mathbb{U}$ be a simply connected domain with $B(0, \epsilon) \subset D$, and let $F_{\epsilon}$ be the connected component of 0 in the set of all points 
$z$ with $d(z, \partial D) \geqslant \epsilon$. Let $\delta \in\left(0, \delta_{1}\right)$, and let $Q \subset \mathrm{V}_{\partial}^{0}(D, \delta)$ be nonempty. Let $\phi$ be the conformal homeomorphism from $\mathbb{U}$ to $D$ that satisfies $\phi(0)=0$ and $\phi^{\prime}(0)>0$. Then there is a random $\lambda \in \partial \mathbb{U}$ independent of $\sigma^{1}$ such that

$$
\widehat{d}_{F_{\epsilon}}\left(\phi\left(\lambda \sigma^{1}\right), \operatorname{LE}\left(\operatorname{RW}^{D, \delta, Q}\right)\right)<\epsilon .
$$

The main point here is that $\delta_{1}$ does not depend on $D$ or on $Q$.

Proof: Let $\epsilon_{1}>0$ be much smaller than $\epsilon$. Suppose that $D^{\prime}$ is a domain in the list appearing in Lemma 5.3 that satisfies the requirements there with $\epsilon_{1}$ in place of $\epsilon$, and let $\nu$ be as in that lemma. Fix some small $\delta_{1}$. For each $q \in \mathrm{V}_{\partial}^{0}\left(D^{\prime}, \delta\right)$ let $\nu_{q}$ be restriction of the hitting measure of $\mathrm{RW}^{D^{\prime}, \delta}$ to $B\left(q, \delta_{1}\right) \cap V_{\partial}(D, \delta)$, normalized to be a probability measure. Lemma 5.4 implies that we may replace $\nu$ by a probability measure $\nu^{\prime}$, which is a convex combination of such $\nu_{q}$, while having

$$
\widehat{d}_{F_{\epsilon_{1}}}\left(\operatorname{LE}\left(\operatorname{RW}^{D^{\prime}, \delta, \nu}\right), \operatorname{LE}\left(\operatorname{RW}^{D^{\prime}, \delta, \nu^{\prime}}\right)\right)<\epsilon_{1},
$$

provided $\delta_{1}$ is sufficiently small. Moreover, by Conjecture 1.2 , provided $\delta_{1}$ is sufficiently small and $\delta \in\left(0, \delta_{1}\right)$, we have

$$
\widehat{d}_{F_{\epsilon_{1}}}\left(\operatorname{LE}\left(\mathrm{RW}^{D^{\prime}, \delta, \nu_{q}}\right), \psi\left(\lambda_{q} \sigma^{1}\right)\right) \leqslant \epsilon_{1},
$$

where $\lambda_{q} \in \partial \mathbb{U}$ is random and independent of $\sigma^{1}$, and $\psi$ is the conformal homeomorphism from $\mathbb{U}$ to $D^{\prime}$ satisfying $\psi(0)=0$ and $\psi^{\prime}(0)>0$. Since the list $D_{1}, \ldots, D_{n}$ in Lemma 5.3 is finite, we may take $\delta_{1}$ to be independent of $D$. Consequently, there is a random $\lambda \in \partial \mathbb{U}$ independent of $\sigma^{1}$ with

$$
\widehat{d}_{F_{\epsilon_{1}}}\left(\operatorname{LE}\left(\operatorname{RW}^{D^{\prime}, \delta, \nu^{\prime}}\right), \psi\left(\lambda \sigma^{1}\right)\right) \leqslant \epsilon_{1} .
$$

Provided we have chosen $\epsilon_{1}$ sufficiently small, we have that $\left|\psi\left(\phi^{-1}(z)\right)-z\right|<\epsilon_{1}$ for $z \in F_{\epsilon / 2}$. Proposition 5.5 now follows from (5.3) with $\epsilon_{1}$ in place of $\epsilon$ and from (5.4), (5.5) and (5.6).

\section{Recognizing the Löwner parameter as Brownian motion}

Proof of Proposition 4.2: Let $\beta:=\mathrm{LE}\left(\mathrm{RW}^{\mathrm{U}, \delta}\right)$ and let $\beta_{t}, D_{t}$ and $q_{1}(t)$ be defined as in Lemma 4.3 , where $D:=\mathbb{U}$. Set $\gamma:=\operatorname{LE}\left(\operatorname{RW}^{D_{t}, \delta, q_{1}(t)}\right)$, where $\mathrm{RW}^{D_{t}, \delta, q_{1}(t)}$ is taken to be independent of $\beta$ conditioned on $\beta_{t}$. Using Conjecture $1.2, \widehat{d}_{\mathrm{U}}(\beta, \sigma) \rightarrow 0$ as $\delta \rightarrow 0$. By (2.1), this means that we may couple $\beta$ and $\sigma$ 
(that is, make them defined on the same probability space, where they are not necessarily independent) such that $\beta \stackrel{\mathbf{P}}{\rightarrow} \sigma$ in $\mathcal{H}(\mathbb{U})$, where $\stackrel{\mathbf{P}}{\rightarrow}$ denotes convergence in probability as $\delta \rightarrow 0$. Since $\sigma$ is a.s. a simple path, this also implies that $\beta_{t} \stackrel{\mathbf{P}}{\rightarrow} \sigma_{t}$.

Let $\widehat{\phi}$ be the conformal map from $\mathbb{U}$ onto $\mathbb{U}-\sigma_{t}$ that satisfies $\widehat{\phi}(0)=0$ and $\widehat{\phi}^{\prime}(0)>0$, and let $\widehat{\psi}$ be the similarly normalized conformal map from $\mathbb{U}$ onto $\mathbb{U}-\beta_{t}$. Because $\beta_{t} \stackrel{P}{\rightarrow} \sigma_{t}$, it follows that $\widehat{\psi} \stackrel{\mathbf{P}}{\rightarrow} \hat{\phi}$, in the topology of uniform convergence on compact subsets of $\mathbb{U}$.

Set $\widehat{\psi}_{\lambda}(z):=\widehat{\psi}(\lambda z)$ and $\widehat{\phi}_{\lambda}(z):=\widehat{\phi}(\lambda z)$ for $\lambda \in \partial \mathbb{U}$. Given every $\epsilon>0$ and a closed set $A \subset \mathbb{U}$ let $F_{\epsilon}(A)$ be the connected component of 0 in the set of points with distance at least $\epsilon$ from $A$ (or the empty set, if $d(0, A)<\epsilon$ ), and let $W_{\epsilon}(A):=\overline{\mathbb{U}-F_{\epsilon}(A)}$.

By Proposition 5.5, for every $\epsilon>0$ there is a random $\lambda \in \partial \mathbb{U}$ independent of $\sigma^{1}$ (but not of $\beta_{t}$ ) such that $\widehat{d}_{F_{\epsilon}\left(\beta_{t}\right)}\left(\widehat{\psi}_{\lambda}\left(\sigma^{1}\right), \gamma\right) \rightarrow 0$. (The law of $\lambda$ may depend on $\delta$ and $\epsilon$.) Observe that $\mathbf{P}\left[F_{2 \epsilon}\left(\sigma_{t}\right) \subset F_{\epsilon}\left(\beta_{t}\right)\right] \rightarrow 1$ as $\delta \rightarrow 0$, because $\beta_{t} \stackrel{\mathbf{P}}{\rightarrow} \sigma_{t}$. Therefore, we may conclude that $\widehat{d}_{F_{2 \epsilon}\left(\sigma_{t}\right)}\left(\widehat{\psi}_{\lambda}\left(\sigma^{1}\right), \gamma\right) \rightarrow 0$. Since this is true for every $\epsilon>0$, it follows that we may choose $\lambda=\lambda_{\delta}$ so that $\widehat{d}_{\mathbb{U}-\sigma_{t}}\left(\widehat{\psi}_{\lambda}\left(\sigma^{1}\right), \gamma\right) \rightarrow 0$, as $\delta \rightarrow 0$. Because $\widehat{\psi}_{\lambda} \stackrel{\mathbf{P}}{\rightarrow} \widehat{\phi}_{\lambda}$, we therefore also have $\widehat{d}_{\mathbf{U}-\sigma_{t}}\left(\widehat{\phi}_{\lambda}\left(\sigma^{1}\right), \gamma\right) \rightarrow 0$, that is, $\widehat{d}_{\mathrm{U}}\left(\sigma_{t} \cup \widehat{\phi}_{\lambda}\left(\sigma^{1}\right), \sigma_{t} \cup \gamma\right) \rightarrow 0$. Since $\beta_{t} \cup \gamma$ has the same law as $\beta$ (by Lemma 4.3), and since $\beta_{t} \stackrel{P}{\rightarrow} \sigma_{t}$, this gives,

$$
\widehat{d}_{\mathbb{U}}\left(\sigma_{t} \cup \widehat{\phi}_{\lambda}\left(\sigma^{1}\right), \beta\right)=\widehat{d}_{\mathbf{U}}\left(\sigma_{t} \cup \widehat{\phi}_{\lambda}\left(\sigma^{1}\right), \beta_{t} \cup \gamma\right) \rightarrow 0 .
$$

Let $\lambda^{*}$ be random in $\partial \mathbb{U}$ with a law that is some weak (subsequential) limit of the law of $\lambda$ as $\delta \rightarrow 0$. It follows from (6.1) that $\sigma_{t} \cup \widehat{\phi}_{\lambda^{*}}\left(\sigma^{1}\right)$ has the same law as $\sigma$. In particular, it is a simple path. The only possibility is therefore that $\widehat{\phi}_{\lambda^{*}}=\phi$ a.s., which completes the proof of Proposition 4.2.

Proof of Proposition 4.1: Recall that $\zeta=\zeta_{\sigma}:(-\infty, 0] \rightarrow \partial \mathbb{U}$ is the Löwner parameter associated to the LERW scaling limit $\sigma \subset \mathbb{U}$. Let $\tilde{\zeta}$ be the Löwner parameter associated with the path $\sigma^{1}$, and let $\tilde{f}_{t}$ be the associated solution of the Löwner system. Note that $\tilde{\zeta}(0)=1$, since $\sigma^{1} \cap \partial \mathbb{U}=\{1\}$. Fix some $t_{0}<0$. Using Proposition 4.2 and its notations (with $t_{0}$ replacing $t$ ), we know that the path $\check{\sigma}:=\sigma_{t_{0}} \cup \phi\left(\sigma^{1}\right)$ has the same law as $\sigma$. Let $\check{f}_{t}$ be the solution of the Löwner system associated with the path $\check{\sigma}$, and let $\check{\zeta}$ be the associated Löwner parameter. Then $\breve{\zeta}$ has the same law as $\zeta$, by Proposition 4.2. Set $\lambda:=\left|\phi^{\prime}(0)\right| / \phi^{\prime}(0)$. When $t<t_{0}$, we have

$$
\check{f}_{t}(z)=\phi \circ \tilde{f}_{t-t_{0}}(\lambda z)
$$


because the right hand side is a suitably normalized conformal map from $\mathbb{U}$ onto $\mathbb{U}-\check{\sigma}_{t}$. We differentiate with respect to $t$, and use (1.1), to get

$$
\begin{aligned}
\frac{\partial}{\partial t} \check{f}_{t}(z) & =\phi^{\prime}\left(\tilde{f}_{t-t_{0}}(\lambda z)\right) \frac{\partial}{\partial t} \tilde{f}_{t-t_{0}}(\lambda z) \\
& =\phi^{\prime}\left(\tilde{f}_{t-t_{0}}(\lambda z)\right) \lambda z \tilde{f}_{t-t_{0}}^{\prime}(\lambda z) \frac{\tilde{\zeta}\left(t-t_{0}\right)+\lambda z}{\tilde{\zeta}\left(t-t_{0}\right)-\lambda z}=z \check{f}_{t}^{\prime}(z) \frac{\lambda^{-1} \tilde{\zeta}\left(t-t_{0}\right)+z}{\lambda^{-1} \tilde{\zeta}\left(t-t_{0}\right)-z} .
\end{aligned}
$$

Consequently, it follows that $\breve{\zeta}(t)=\lambda^{-1} \tilde{\zeta}\left(t-t_{0}\right)$ for $t<t_{0}$. It is clear that $\check{\zeta}=\zeta$ for $t \in\left[t_{0}, 0\right]$. Continuity of $\check{\zeta}$ gives $\lambda^{-1}=\zeta\left(t_{0}\right) / \tilde{\zeta}(0)=\zeta\left(t_{0}\right)$. Since $\zeta$ and $\tilde{\zeta}$ are independent, and $\tilde{\zeta}$ has the same law as $\zeta$ conditioned on $\zeta(0)=1$, Proposition 4.1 follows.

We shall need the following

Theorem 6.1: Let $a(t), t \geqslant 0$, be a real valued process (that is, a random function $a:[0, \infty) \rightarrow \mathbb{R}$ ). Suppose that $a$ is continuous a.s. and for every $n \in \mathbb{N}$ and every $(n+1)$-tuple $0=t_{0} \leqslant t_{1} \leqslant t_{2} \leqslant \cdots \leqslant t_{n}$, the increments $a\left(t_{j}\right)-a\left(t_{j-1}\right)$, $j=1, \ldots, n$, are independent. Then for every fixed $s_{0} \in(0, \infty)$, the random variable $a\left(s_{0}\right)$ is Gaussian.

This theorem follows from the general theory of Lévy processes. An entirely elementary proof can be found in Section 4.2 of [Itô61].

Corollary 6.2: There is a constant $c>0$ such that the process $\zeta(t)$ has the same law as $\mathrm{B}(-c t)$, where $\mathrm{B}(t)$ is Brownian motion on $\partial \mathbb{U}$ started at a uniform random point.

Proof: That $\zeta(t)$ has the same law as $\mathrm{B}(-c t)$ for some $c \geqslant 0$ follows immediately from Proposition 4.1 and Theorem 6.1. The fact that $c>0$ is clear, since the LERW scaling limit is not equal a.s. to a line segment.

\section{The winding number of SLE}

Let $\kappa \geqslant 0$, let $\mathrm{B}(t)$ be Brownian motion on $\partial \mathbb{U}$ started at a uniform random point on $\partial \mathbb{U}$, and set

$$
\zeta=\zeta_{\kappa}:=\mathrm{B}(-\kappa t)
$$

Definition 7.1: Let $\mathfrak{K}$ denote the set of all $\kappa \geqslant 0$ such that the Löwner evolution $f_{t}$ defined by (7.1), (1.1) and (1.2) is a.s. for every $t<0$ a Riemann map to a slitted disk. For $\kappa \in \mathfrak{K}$, let $\xi_{\kappa}$ denote the (random) path defined by $\xi_{\kappa}(t)=$ 
$f_{t}(\zeta(t))$. That is, $\xi_{\kappa}$ is the path in $\overline{\mathbb{U}}$ such that $f_{t}$ is the nomalized Riemann map to $\mathbb{U}-\xi_{\kappa}([t, 0])$.

The random process $\xi_{\kappa}([t, 0]), t \leqslant 0$, will be called stochastic Löwner evolution (SLE) with constant $\kappa$.

As before, we let $\widehat{\mathrm{B}}:[0, \infty) \rightarrow \mathbb{R}$ be the continuous map satisfying $\mathrm{B}=\exp i \widehat{\mathrm{B}}$ and $\widehat{B}(0) \in[0,2 \pi)$.

TheOrem 7.2: Let $\kappa \in \mathfrak{K}$. Let $T \leqslant 0$, and let $\theta_{\kappa}(T)$ be the winding number of the path $\xi_{\kappa}([T, 0])$ around 0 , that is $\theta_{\kappa}(T)=\arg \left(\xi_{\kappa}(0)\right)-\arg \left(\xi_{\kappa}(T)\right)$, with $\arg$ chosen continuous along $\xi_{\kappa}$. Then for all $s>0$,

$$
\mathbf{P}\left[|T-\log | \xi_{\kappa}(T)||>s\right] \leqslant C_{0} \exp \left(-C_{1} s\right),
$$

and

$$
\mathbf{P}\left[\left|\theta_{\kappa}(T)-\widehat{\mathrm{B}}(0)+\widehat{\mathrm{B}}(-\kappa T)\right|>s\right] \leqslant C_{0} \exp \left(-C_{1} s\right),
$$

where $C_{0}, C_{1}>0$ are constants, which depend only on $\kappa$.

Loosely speaking, the theorem says that $t+i \widehat{\mathrm{B}}(-\kappa t)$ is a good approximation of the path $\log \xi_{\kappa}(t)$. A consequence of the theorem is that $\theta_{\kappa}(t) / \sqrt{\kappa t}$ converges to a gaussian of unit variance as $T \rightarrow-\infty$.

Before we prove the theorem, it is worthwhile to have another look at the maps $\Phi$ defined by $\Phi(z, t, s):=f_{s}^{-1}\left(f_{t}(z)\right), t \leqslant s \leqslant 0$, which were discussed in Remark 1.5. For every $t<s \leqslant 0$ the set $\gamma_{t}^{s}:=\mathbb{U}-\Phi(\mathbb{U}, t, s)$ is a path in $\mathbb{U}$. If $r \in(t, s)$, then $\gamma_{t}^{s}=\gamma_{r}^{s} \cup \Phi\left(\gamma_{t}^{r}, r, s\right)$. Therefore, when $s$ is held fixed and $t$ is decreasing, the path $\gamma_{t}^{s}$ is growing from its endpoint inside $\mathbb{U}$, and if $t$ is held fixed and $s$ is increasing, the path $\gamma_{t}^{s}$ deforms conformally and a new arc is added to it to reconnect it to $\partial \mathbb{U}$.

Proof: Let $f_{t}$ be defined by (7.1), (1.1) and (1.2). Set $\xi:=\xi_{\kappa}$. Let $w(t, z):=$ $f_{t}^{-1}\left(f_{T}(z)\right)=\Phi(z, T, t)(t \in[T, 0])$, and let $y=y(t, z):=\arg w(t, z)$, where $\arg w$ is chosen to be continuous in $t$.

By Remark 1.5, $w$ satisfies the differential equation

$$
\partial_{t} w=-w \frac{\mathrm{B}(-\kappa t)+w}{\mathrm{~B}(-\kappa t)-w}
$$

where $\partial_{t}$ denotes differentiation with respect to $t$. Set $x=x(t, z):=\log |w(t, z)|$. Then $w=\exp (x+i y)$, and (7.4) can be rewritten

$$
\partial_{t} x+i \partial_{t} y=\frac{\sinh x+i \sin (\widehat{\mathrm{B}}(-\kappa t)-y)}{\cosh x-\cos (\widehat{\mathrm{B}}(-\kappa t)-y)} .
$$


Let $z_{1}$ be a random point on $\partial \mathbb{U}$, chosen uniformly, and independent of the Brownian motion $B$. Then $w\left(0, z_{1}\right)=f_{T}\left(z_{1}\right)$ is some point on the boundary of $D_{T}:=f_{T}(\mathbb{U})$. Note that $\partial D_{T}$ is a connected set that contains $\partial \mathbb{U}$ and intersects the circle $\partial B(0, \exp (T))$, by (2.2). Set $A_{s}:=\left\{z \in \partial D_{T}:|z|>\exp (T+s)\right\}$. It follows from the continuous version of Lemma 2.1 for Brownian motion that the harmonic measure of $A_{s}$ in $D_{T}$ at 0 is bounded by $O(1) \exp \left(-C_{2} s\right)$, for some constant $C_{2}>0$ and every $s \in \mathbb{R}$; that is, at zero, the bounded harmonic function on $D_{T}$ that has boundary values 1 on $A_{s}$ and has boundary values 0 on $\partial D_{T}-\bar{A}_{s}$ is bounded from above by $O(1) \exp \left(-C_{2} s\right)$. Since harmonic measure is invariant under conformal maps, we conclude that the measure of $f_{T}^{-1}\left(A_{s}\right)$ is at most $O(1) \exp \left(-C_{2} s\right)$. This means that

$$
\mathbf{P}\left[\log \left|w\left(0, z_{1}\right)\right|-T>s\right]=\mathbf{P}\left[\log \left|f_{T}\left(z_{1}\right)\right|-T>s\right] \leqslant O(1) \exp \left(-C_{2} s\right) .
$$

Now set $z_{0}=\mathrm{B}(-\kappa T)$. Then $\xi(T)=w\left(0, z_{0}\right)$, and so we need to relate $\left|w\left(0, z_{0}\right)\right|$ and $\left|w\left(0, z_{1}\right)\right|$. Let $\tau$ be the least $t \in[T, 0]$ such that $w\left(t, z_{1}\right)=\mathrm{B}(-\kappa t)$, if such a $t$ exists, and set $\tau=0$ if not. Note that $\left|w\left(t, z_{1}\right)\right|=1$ while $t<\tau$, and $\left|w\left(t, z_{1}\right)\right|<1$ for $t \in(\tau, 0]$. Also observe that conditioned on $\tau<0$, the law of the process $\left(w\left(t, z_{1}\right): t \in[\tau, 0]\right)$ is the same as the law of the process $\left(w\left(t+T-\tau, z_{0}\right): t \in[\tau, 0]\right)$. Consequently, the random variable $w\left(\tau-T, z_{1}\right)$ (where B is taken as two-sided Brownian motion and (7.4) is extended to the range $t>0$ ), conditioned on $\tau<0$, has the same distribution as the random variable $w\left(0, z_{0}\right)$. By $(7.5), \partial_{t} x \leqslant 0$, and therefore $\left|w\left(\tau-T, z_{1}\right)\right| \leqslant\left|w\left(0, z_{1}\right)\right|$ on the event $\tau<0$. Thus, for every $s \in \mathbb{R}$ we have

$$
\mathbf{P}\left[\left|w\left(0, z_{1}\right)\right|>s \mid \tau<0\right] \geqslant \mathbf{P}\left[\left|w\left(0, z_{0}\right)\right|>s\right] .
$$

Because $\left|w\left(0, z_{1}\right)\right|=1$ when $\tau=0$, we may drop the conditioning on $\tau<0$. Now (7.6) gives

$$
\mathbf{P}[\log |\xi(T)|-T>s]=\mathbf{P}\left[\log \left|w\left(0, z_{0}\right)\right|-T>s\right] \leqslant O(1) \exp \left(-C_{2} s\right) .
$$

On the other hand, the Koebe $1 / 4$ Theorem (2.3) gives

$$
\exp T=\left|f_{T}^{\prime}(0)\right| \leqslant 4 \inf \left\{|z|: z \notin f_{T}(\mathbb{U})\right\} \leqslant 4|\xi(T)|,
$$

and so $\log |\xi(T)|+\log 4 \geqslant T$ always. This completes the proof of (7.2).

Now let $\tau_{1}$ be the least $t \in[T, 0]$ such that $x(t)=\log \left|w\left(t, z_{0}\right)\right| \leqslant-1$, and set $\tau_{1}:=0$ if such a $t$ does not exist. Since $x(t)$ is monotone decreasing, we may write $y(t)$ as a function of $x: y=g(x)$. By (7.5),

$$
g^{\prime}(x(t))=\frac{\sin (\widehat{\mathrm{B}}(-\kappa t)-y(t))}{\sinh x(t)}
$$


and hence

$$
\left|g^{\prime}(x(t))\right| \leqslant|\sinh x(t)|^{-1}
$$

And so we get

$$
\left|y(0)-y\left(\tau_{1}\right)\right|=\int_{x(0)}^{x\left(\tau_{1}\right)}\left|g^{\prime}(x)\right| d x \leqslant \int_{-\infty}^{-1}|\sinh x|^{-1} d x<\infty .
$$

Let $\phi(s, t):=f_{t}^{-1}(\xi(s))$ for $T \leqslant s \leqslant t \leqslant 0$. Then $\phi$ is continuous and its image does not contain 0 . Hence, it may be considered as a homotopy in $\mathbb{C}-\{0\}$ from the path $\phi(s, 0)=\xi(s), s \in[T, 0]$, to the concatenation of the inverse of the path $\phi(T, t)=f_{t}^{-1}(\xi(T))=w\left(t, z_{0}\right), t \in[T, 0]$, with the path $\phi(t, t)=\mathrm{B}(-\kappa t)$, $t \in[T, 0]$. Therefore, its winding number is the sum of the corresponding winding numbers. This means that

$$
\theta_{\kappa}(T)=\widehat{\mathrm{B}}(0)-\widehat{\mathrm{B}}(-\kappa T)+y(T)-y(0) .
$$

By (7.7), it therefore suffices to prove the appropriate bound on the tail of $\left|y\left(\tau_{1}\right)-y(T)\right|$.

Let $|y|_{2 \pi}:=\min \{|y-2 \pi n|: n \in \mathbb{Z}\}$. Set $t_{0}=T$, and inductively, let $t_{j}$ be the first $t \in\left[t_{j-1}, 0\right]$ such that $\pi / 2=\left|\widehat{\mathrm{B}}(-\kappa t)-\widehat{\mathrm{B}}\left(-\kappa t_{j-1}\right)\right|_{2 \pi}$, and set $t_{j}=$ 0 if no such $t$ exists. Equation (7.5) shows that $\partial_{t} y(t)$ has the same sign as $\sin (\widehat{B}(-\kappa t)-y(t))$, and hence

$$
\partial_{t}|y(t)-\widehat{\mathrm{B}}(-\kappa s)|_{2 \pi} \leqslant 0, \quad \text { at } t=s,
$$

for every $s \in(T, 0)$. In other words, $y(t)$ is always moving in the direction which would decrease $|\exp (i y(t))-\mathrm{B}(-\kappa t)|$. Consequently, for every $j \in \mathbb{N}$, if there is an $s \in\left[t_{j}, t_{j+1}\right]$ such that $\left|y(s)-\widehat{\mathrm{B}}\left(-\kappa t_{j}\right)\right|_{2 \pi}<\pi / 2$, then this is satisfied also for all $s^{\prime} \in\left[s, t_{j+1}\right)$, because $y(t)^{2 \pi}$ cannot get out of the set $\left\{p \in \mathbb{R}:\left|p-\widehat{\mathrm{B}}\left(-\kappa t_{j}\right)\right|_{2 \pi}<\pi / 2\right\}$ while $\widehat{\mathrm{B}}(-\kappa t)$ is in it. This implies that $\left|y\left(t_{j+1}\right)-y\left(t_{j}\right)\right|<2 \pi$. Hence $\left|y\left(\tau_{1}\right)-y(T)\right| \leqslant 2 \pi \min \left\{j \in \mathbb{N}: t_{j}>\tau_{1}\right\}$. Therefore, for every $a>0$ and $n \in \mathbb{N}$,

$$
\mathbf{P}\left[\left|y\left(\tau_{1}\right)-y(T)\right| \geqslant 2 \pi n\right] \leqslant \mathbf{P}\left[\tau_{1}-T \geqslant a\right]+\mathbf{P}\left[t_{n} \leqslant T+a\right] .
$$

The first summand on the right hand side is bounded by $O(1) \exp \left(-C_{3} a\right)$, for some constant $C_{3}>0$, by (7.2). To estimate the second summand, observe that conditioned on $t_{n} \leqslant T+a$, we have probability at least $2^{-n-1}$ for the event

$$
B(-\kappa(T+a))-\widehat{\mathrm{B}}(-\kappa T) \geqslant n \pi / 2,
$$


because when $t_{n} \leqslant T+a$ and $B(-\kappa(T+a)) \geqslant B\left(-\kappa t_{n}\right) \geqslant \cdots \geqslant B\left(-\kappa t_{0}\right)$, we have (7.9). However, (7.9) has probability

$$
(2 \pi a \kappa)^{-1 / 2} \int_{n \pi / 2}^{\infty} \exp \left(-s^{2} /(2 \kappa a)\right) d s \leqslant O(1) \exp \left(-n^{2} / C_{4} a\right)
$$

and hence

$$
\mathbf{P}\left[t_{n} \leqslant T+a\right] \leqslant O(1) 2^{n} \exp \left(-n^{2} / C_{4} a\right) .
$$

We choose $a$ to be $n$ times a very small constant. Then our above estimates, together with (7.8), give

$$
\mathbf{P}\left[\left|y\left(\tau_{1}\right)-y(T)\right| \geqslant 2 \pi n\right] \leqslant O(1) \exp \left(-C_{5} n\right),
$$

with the constants depending only on $\kappa$. This completes the proof of the theorem.

\section{The twisting constant of LERW}

Consider some scaling limit measure $\mathbf{P}$ of LERW from 0 to $\partial \mathbb{U}$, and let $\gamma$ be random with law P. Assuming Conjecture 1.2, we have established that SLE with some constant $\kappa_{o}$ has law $\mathbf{P}$. In this section we show that $\kappa_{o}=2$, and thereby complete the proof of Thereom 1.3.

Let $\epsilon \in(0,1)$, let $\gamma_{\epsilon}$ be the connected component of $\gamma-B(0, \epsilon)$ which has a point in $\partial U$, and let $W\left(\gamma_{\epsilon}, 0\right)$ be the winding number of $\gamma_{\epsilon}$ around 0 , in radians. That is, $W\left(\gamma_{\epsilon}, 0\right)$ is the imaginary part of $\int_{\gamma_{\epsilon}} z^{-1} d z$. By symmetry, it is clear that $\mathbf{E}\left[W\left(\gamma_{\epsilon}, 0\right)\right]=0$. We shall show that

$$
\mathbf{E}\left[W\left(\gamma_{\epsilon}, 0\right)^{2}\right]=2 \log (1 / \epsilon)+O(1) \sqrt{\log (1 / \epsilon)} .
$$

Based on this and the results of Section 7, it will follow that $\kappa_{o}=2$.

The proof of (8.1) will use Kenyon's work [Ken98a]. The overall idea of the proof is very simple, and based on the relations between UST and domino tilings. We now briefly review the relations between the UST on $\mathbb{Z}^{2}$ and domino tilings, and the height function for domino tilings. For a more thorough discussion, the reader should consult [Ken98a].

A domino tiling of the grid $\mathbb{Z}^{2}$ is a tiling of $\mathbb{R}^{2}$ by tiles of the forms $[k, k+1] \times[j, j+2]$ and $[k, k+2] \times[j, j+1]$, where $k, j \in \mathbb{Z}$. A domino tiling of $\mathbb{Z}^{2}$ may also be thought of as a perfect matching of the dual grid $((1 / 2)+\mathbb{Z})^{2}$. (A perfect matching of a graph $G$ is a set of edges $M \subset \mathrm{E}(G)$ such that every vertex is incident with precisly one edge in $M$.) 
Let us start with finite graphs. Let $D$ be a simply connected domain in $\mathbb{R}^{2}$ whose boundary is a simple closed curve in the grid $\mathbb{Z}^{2}$, and let $G:=\mathbb{Z}^{2} \cap \bar{D}$. Let $\rho_{0}$ be some vertex in $\partial D \cap \mathbb{Z}^{2}$, which we call the root. Let $\check{G}$ be the graph $\left((1 / 2) \mathbb{Z}^{2}\right) \cap \bar{D}$ with $\rho_{0}$ and its incident edges removed. Then there is a bijection, discovered by Temperley, between the set of perfect matchings on $\breve{G}$ and spanning trees of $G$.

Temperley's bijection (see Figure 8.1) works as follows. For every edge $[v, u]$ in the matching $M$ such that $v \in \mathbb{Z}^{2}$, we put in the tree the edge $e_{u}$ whose center is $u$. This gives the set of edges in the tree $T$. If $[v, u] \in M$ is as above, we may orient the edge $e_{u}$ away from $v$, and then the tree $T$ will be oriented towards the $\operatorname{root} \rho_{0}$.

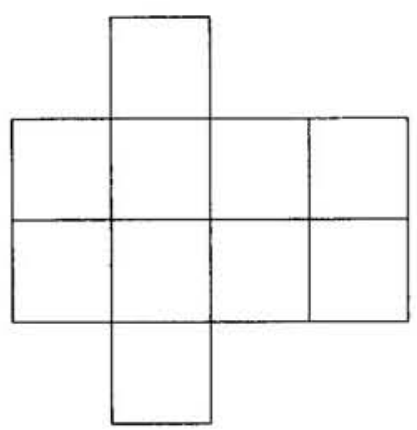

$\rho_{0} \quad G$

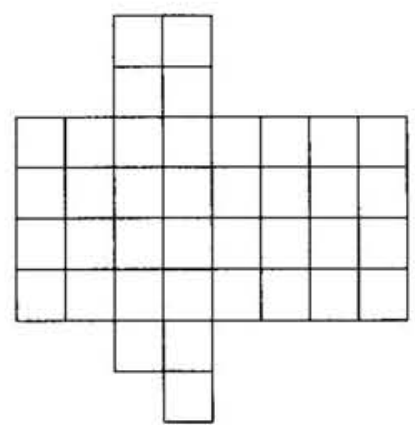

$\check{G}$

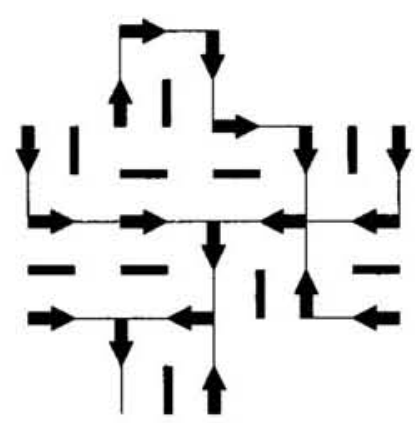

matching and tree

Figure 8.1. Temperley's bijection. On the right, the arrows are edges in the matching that contains vertices of $\mathbb{Z}^{2}$, the solid segments are other edges in the matching, and the thin lines are edges in the tree.

Temperley's bijection works also in more general situations. There is a simple modification to make it work for the wired graph associated to the domain $D$ (recall the notion of the wired graph from Section 2). Also, given a perfect matching on all of $(1 / 2) \mathbb{Z}^{2}$, there is an associated (oriented) spanning forest of $\mathbb{Z}^{2}$. The collection of all domino tilings of $\mathbb{Z}^{2}$ has a natural stationary probability measure (of maximal entropy), and for a.e. domino tiling the corresponding spanning forest is a spanning tree. Temperley's map from perfect matchings on $(1 / 2) \mathbb{Z}^{2}$ to spanning forests of $\mathbb{Z}^{2}$ maps the cannonical probability measure on the set of domino tilings to the law of the UST of $\mathbb{Z}^{2}$.

Let $G$ and $\breve{G}$ be as above, and let $\widehat{G}$ be the graph of the domino tiling, that is, the union of the squares of edge length $1 / 2$ with centers at the vertices of 
$\breve{G}$, thought of as a subgraph of the grid $(1 / 4,1 / 4)+(1 / 2) \mathbb{Z}^{2}$. Associated to a domino tiling of $\widehat{G}$ is a height function $h$ defined on the vertices of $\widehat{G}$. Here is the definition of $h$. Pick some vertex $v_{0} \in \mathrm{V}(\widehat{G})$ and some $a_{0} \in \mathbb{R}$, and set $h\left(v_{0}\right)=a_{0}$. Color a square face of the grid $(1 / 4,1 / 4)+(1 / 2) \mathbb{Z}^{2}$ white if its center is a vertex of $\mathbb{Z}^{2}$ or if it is contained in a face of $\mathbb{Z}^{2}$, and black otherwise. If $[u, v] \in \mathrm{E}(\widehat{G})$ is on the boundary of a domino tile in the tiling, then we require that $h(v)-h(u)=1$ if the square to the right of the directed edge $[u, v]$ is white and $h(v)-h(u)=-1$ if the square to the right of $[u, v]$ is black. These constraints uniquely specify the height function $h$ (except that the choices of $v_{0}$ and $a_{0}$ are arbitrary).

We will work in the upper half plane $\mathbb{H}:=\{z \in \mathbb{C}: \operatorname{Im} z>0\}$. Let $G_{\delta}(\mathbb{H}):=$ $G^{W}(\mathbb{H}, \delta)$, the wired graph of mesh $\delta$ associated with the domain $\mathbb{H}$, and $\widehat{G}_{\delta}(\mathbb{H}):=$ $\mathbb{H} \cap\left((\delta / 4, \delta / 4)+(\delta / 2) \mathbb{Z}^{2}\right)$. The discussion above carries through for the grid $\delta \mathbb{Z}^{2}$, in place of $\mathbb{Z}^{2}$. (Although the distance between adjacent vertices in the graph $\widehat{G}$ is $\delta / 2$ when $G \subset \delta \mathbb{Z}^{2}$, we still work with the height function where the height difference along an edge on the boundary of a tile is \pm 1 .) Temperley's bijection induces a measure preserving transformation between domino tilings of the grid $\widehat{G}_{\delta}(\mathbb{H})$ and the UST of $G_{\delta}(\mathbb{H})$. (If we keep the orientation, then the UST is directed towards $\partial \mathbb{H}$.)

We normalize the height function associated to a domino tiling of $\widehat{G}_{\delta}(\mathbb{H})$ by requiring that $h((\delta / 4, \delta / 4))=1 / 2$. Then $h(((2 k+1) \delta / 4, \delta / 4))=(-1)^{k} / 2$, for $k \in \mathbb{Z}$. If $v$ is some vertex in $G_{\delta}(\mathbb{H})$, let $h(v)$ be the average of the value of $h$ on the vertices of $\widehat{G}_{\delta}(\mathbb{H})$ closest to $v$.

LEMMA 8.1: Let $T$ be a spanning tree of $G_{\delta}(\mathbb{H})$, and let $h$ be the associated height function. Let $v \in \mathrm{V}\left(G_{\delta}(\mathbb{H})\right)$ be a vertex different from the wired vertex $\partial \mathbb{H}$, and let $a$ be the real part of $v$. Let $Q_{v}^{\prime}$ be the path from $v$ to $\partial \mathbb{H}$ in $T$, considered as a path in the plane, and let $Q_{v}$ be the union of $Q_{v}^{\prime}$ with the line segment joining the intersection $Q_{v}^{\prime} \cap \partial \mathbb{H}$ to $a$. Then $-\pi h(v) / 2=W\left(Q_{v}, v\right)$, the winding number of $Q_{v}^{\prime}$ around $v$.

This lemma is a special case of a more general observation made by Kenyon. (Since $Q_{v}$ is a path with $v$ as an endpoint, we define

$$
W\left(Q_{v}, v\right):=\lim _{r \rightarrow 0} W\left(Q_{v}-B(v, r), v\right),
$$

which is the same as $W\left(Q_{v}-B(v, \delta / 2), v\right)$.)

Proof: Use induction on the length of the path $Q_{v}^{\prime}$. 
Symmetry implies that $\mathbf{E}[h(v)]=0$ for all $v \in \mathrm{V}\left(G_{\delta}(\mathbb{H})\right)$. Kenyon has shown [Ken98a] that

$$
\mathbf{E}\left[h(v)^{2}\right]=8 \pi^{-2} \log (1 / \delta)+O(1)
$$

(provided that $v$ stays in a compact subset of $\mathbb{H}$ ). Hence $\mathbf{E}\left[W\left(Q_{v}, v\right)^{2}\right]=$ $2 \log (1 / \delta)+O(1)$, which seems very close to a proof of (8.1). However, to make it into a proof of (8.1) requires some effort (it seems).

The advantage of the height function over the winding number is that the height difference between two vertices can be computed along any path joining them. On the other hand, to compute $W\left(Q_{v}, v\right)$, one might think that it is necessary to follow $Q_{v}$, which is a random path. It is immediate that $h(v)$ is $\sum_{j} \lambda_{j} \chi_{j}$, where $\chi_{j}$ is the indicator of the event that a certain domino tile is present in the tiling, and $\lambda_{j}$ are some explicit easy to compute (non-random) weights. This means that to calculate $\mathbf{E}\left[h(v)^{2}\right]$ one needs to have a good estimate for the behavior of the correlations $\mathbf{E}\left[\chi_{i} \chi_{j}\right]$ for small $\delta$. That's how Kenyon proves (8.2).

Recall that $A\left(p, r_{1}, r_{2}\right)$ denotes an annulus with center $p$, inner radius $r_{1}$, and outer radius $r_{2}$. The following result is an immediate consequence of [ABNW].

Lemma 8.2: Let $D \subset \mathbb{C}$ be a domain, and let $v_{0} \in \delta \mathbb{Z}^{2} \cap D$ be some vertex. Consider $T$, the UST on $\delta \mathbb{Z}^{2} \cap D$, with free or wired boundary. Let $r_{2}>2 r_{1}>2 \delta$, and suppose that $r_{2}$ is smaller than the distance from $v_{0}$ to $\partial D$. Let $A$ be the annulus $A:=A\left(v_{0}, r_{1}, r_{2}\right)$, and let $k(A)$ be the maximum number of disjoint paths in $T$ each of which intersects both boundary components of $A$. Then for each $k \in \mathbb{N}$

$$
\mathbf{P}[k(A) \geqslant k] \leqslant C_{0}\left(r_{2} / r_{1}\right)^{C_{1}(k-1)},
$$

where $C_{0}, C_{1}>0$ are universal constants.

Lemma 8.3: Let $D \subset \mathbb{C}$ be some simply connected domain, and consider $T$, the UST in $G^{W}(D, \delta)$ (wired boundary). Let $p_{0} \in D \cap \delta \mathbb{Z}^{2}$. Given a set $K \subset D$, let $X(K)$ denote the maximum winding number around $p_{0}$ of a path in $T$ with endpoints in $K$. Let $r \in\left(0, d\left(p_{0}, \partial D\right) / 2\right)$. Then for each $h>0$ and $s \geqslant 2$,

$$
\mathbf{P}\left[X\left(A\left(p_{0}, r / s, r\right)\right)>h\right] \leqslant C_{2} \exp \left(-C_{3} h / \log s\right) \log s,
$$

where $C_{2}$ and $C_{3}$ are absolute constants.

Proof: Set $A:=A\left(p_{0}, r / s, r\right), X:=X(A)$. To begin, assume that $s=2$ and that $r>10 \delta$. Let $B_{1}, B_{2}, \ldots, B_{N}$ be a covering of $A$ with balls of radius $r / 10$, where 
$N \leqslant C_{4}$, with $C_{4}$ some universal constant. Let $v_{0} \in A \cap \delta \mathbb{Z}^{2}$ be some vertex. For any vertex $u$, let $W(u)$ be the signed winding number around $p_{0}$ of the path in $T$ from $v_{0}$ to $u$. Consider neighbors $v, w$ in $A$, and let $\gamma$ be the UST path joining $v$ and $w$ in $T$. If $\gamma \neq[v, w]$, then $\gamma \cup[v, w]$ is a simple closed path, and hence has winding number at most $2 \pi$ around $p_{0}$. This implies that $|W(v)-W(u)|$ is at most $2 \pi$ plus the absolute value of the winding number of the edge $[v, u]$ around $p_{0}$, and therefore $|W(v)-W(u)| \leqslant 3 \pi$. Since any two vertices in $A$ can be connected by a path in $A$, the set $\{W(v): v \in A\}$ intersects every interval of length $3 \pi$ which lies between its maximum and minimum. From this it follows that we may find vertices $v_{1}, v_{2}, \ldots, v_{n}$ in $A$ such that $\left|W\left(v_{j}\right)-W\left(v_{k}\right)\right| \geqslant 3 \pi$ for $j \neq k$ and $n \geqslant X / 6 \pi$. Since $N \leqslant C_{4}$, we may find some ball $B_{m}$ from the above collection, satisfying $\left|B_{m} \cap\left\{v_{1}, \ldots, v_{n}\right\}\right| \geqslant X / 6 \pi C_{4}$. However, if $v, u \in B_{m}$ and $|W(v)-W(u)| \geqslant 2 \pi$, then the path in $T$ joining $v$ and $u$ must go around $p_{0}$. In particular, it must cross twice the annulus $A_{m}:=A\left(c_{m}, r / 10, r / 5\right)$, where $c_{m}$ is the center of $B_{m}$. It follows that the number of disjoint crossings of $A_{m}$ in $T$ is at least $\left|B_{m} \cap\left\{v_{1}, \ldots, v_{n}\right\}\right|$. Hence, by Lemma 8.2 , for any fixed $m$ the probability that $\left|B_{m} \cap\left\{v_{1}, \ldots, v_{n}\right\}\right|>b$ is at most $O(1) \exp \left(-C_{5} b\right)$, for some constant $C_{5}>0$. Consequently, $\mathbf{P}[X>h] \leqslant O(1) C_{4} \exp \left(-C_{5} h / 6 \pi C_{4}\right)$, which completes the proof in the case $s=2$ and $r>10 \delta$.

The case $r \leqslant 10 \delta$ is easy to verify, for then the combinatorial distance between any two vertices in $A$ is bounded, which implies that $X$ is bounded (because $|W(v)-W(u)| \leqslant 3 \pi$ for neighbors $v, u)$.

If $s>2$, then we may cover the annulus $A$ with at most $2 \log s+1$ disjoint concentric annuli with radii ratio 2 . In order that $X(A)$ be at least $h$, there must be one of these smaller annuli $A^{\prime}$ with $X\left(A^{\prime}\right) \geqslant h /(2 \log s+1)$. The lemma follows.

Lemma 8.4 ([Ken98a]): Let $p, q \in \mathbb{H}$ be any two points. Consider a uniform domino tiling of the grid $\widehat{G}_{\delta}(\mathbb{H}) \subset \mathbb{H}$ of mesh $\delta$, and let $p^{\prime}, q^{\prime} \in \mathrm{V}\left(\widehat{G}_{\delta}(\mathbb{H})\right)$ be vertices closest to $p$ and $q$, respectively. Then

$$
\lim _{\delta \rightarrow 0} \mathbf{E}\left[h\left(p^{\prime}\right) h\left(q^{\prime}\right)\right]=8 \pi^{-2} \log \left|\frac{\bar{p}-q}{p-q}\right| .
$$

It may be noted that the right hand side is invariant under conformal automorphisms of $\mathbb{H}$, since it is the log of the square root of a cross ratio of $p, \bar{p}, q, \bar{q}$.

Let $\delta \in(0,1 / 4)$, let $v_{0}$ be a vertex of the grid $\delta \mathbb{Z}^{2}$ which is closest to $i=\sqrt{-1}$. Fix some $r \in(0,1 / 4)$. Let $Q_{v}$ be as in Lemma 8.1, let $\gamma_{r}^{\delta}$ be the connected 
component of $Q_{v_{0}}-B\left(v_{0}, r\right)$ that intersects $\partial \mathbb{H}$, and let $W_{\delta}(r)$ be the winding number of $\gamma_{r}^{\delta}$ around $v_{0}$.

Proposition 8.5: Assuming $r<1 / 4$,

$$
\limsup _{\delta \rightarrow 0}\left|\mathbf{E}\left[W_{\delta}(r)^{2}\right]-2 \log (1 / r)\right| \leqslant C_{6} \sqrt{\log (1 / r)},
$$

where $C_{6}$ is an absolute constant.

Proof: Let $v_{1}$ be a vertex in $\delta \mathbb{Z}^{2}$ such that $v_{1}-v_{0}-r / 3 \in[0, \delta)$. Let $V_{0}$ be the set of vertices of $\delta \mathbb{Z}^{2}$ whose Euclidean distance to $\left\{v_{1}, v_{0}\right\}$ is in the range $(r / 9,2 r)$. Then, assuming that $\delta<r / 9, V_{0}$ separates $v_{0}$ from $v_{1}$ and $\left\{v_{0}, v_{1}\right\}$ from $\partial \mathbb{H}$ in the grid $\delta \mathbb{Z}^{2}$. Given a vertex $v$, let $Q_{v}^{0}$ be the union of $Q_{v}^{\prime}$ with the line segment joining the intersection $Q_{v}^{\prime} \cap \partial \mathbb{H}$ to 0 . Set $Q:=\bigcup\left\{Q_{v}^{0}: v \in V_{0}\right\}$.

Set $X_{j}:=W\left(Q_{v_{j}}, v_{j}\right), j=0,1$. By Lemma 8.1, we have $X_{j}=-\pi h\left(v_{j}\right) / 2$, and consequently, Lemma 8.4 gives

$$
\lim _{\delta \rightarrow 0} \mathbf{E}\left[X_{0} X_{1}\right]=2 \log (1 / r)+O(1) .
$$

Since $V_{0}$ separates $v_{0}$ from $v_{1}$, when conditioning on $Q, X_{0}$ becomes independent of $X_{1}$. Therefore,

$$
\mathbf{E}\left[X_{0} X_{1}\right]=\mathbf{E}\left[\mathbf{E}\left[X_{0} X_{1} \mid Q\right]\right]=\mathbf{E}\left[\mathbf{E}\left[X_{0} \mid Q\right] \cdot \mathbf{E}\left[X_{1} \mid Q\right]\right] .
$$

We shall show that for small $\delta>0$,

$$
\mathbf{E}\left[\left(W_{\delta}(r)-\mathbf{E}\left[X_{j} \mid Q\right]\right)^{2}\right]=O(1), \quad j=0,1 .
$$

Using (8.5), this implies

$$
\mathbf{E}\left[W_{\delta}(r)^{2}\right]-\mathbf{E}\left[X_{0} X_{1}\right]=O(1) \sqrt{\mathbf{E}\left[W_{\delta}(r)^{2}\right]}+O(1) .
$$

Consequently, by (8.4),

$$
\limsup _{\delta \rightarrow 0}\left|\mathbf{E}\left[W_{\delta}(r)^{2}\right]-2 \log (1 / r)+O(1) \sqrt{\mathbf{E}\left[W_{\delta}(r)^{2}\right]}\right| \leqslant O(1),
$$

which implies (8.3). It therefore suffices to prove (8.6).

Let $s:=\min \left\{\left|v-v_{0}\right|: v \in Q\right\}$. Given $Q$, let $T^{\prime}$ be a UST of $G^{W}\left(B\left(v_{0}, s / 2\right), \delta\right)$, and let $T^{\prime \prime}$ be the UST of the graph obtained from $G_{\delta}(\mathbb{H})$ by identifying the vertices of $Q$. Note that (by Wilson's algorithm, say) $T$, the UST on $G_{\delta}(\mathbb{H})$, has the same law as $T^{\prime \prime} \cup Q$ (as a set of edges). By the domination principle, given 
$Q$, we may couple $T^{\prime \prime}$ and $T^{\prime}$ so that $\mathrm{E}\left(T^{\prime \prime}\right) \supset \mathrm{E}\left(T^{\prime}\right)$. Given $Q$, we couple $T$ and $T^{\prime}$ so that $T \supset T^{\prime}$.

Let $\alpha$ be the path in $T^{\prime}$ from $v_{0}$ to $\partial B\left(v_{0}, s\right)$, and let $a$ be the point where $\alpha$ hits $\partial B\left(v_{0}, s\right)$. Then $\mathbf{E}\left[W\left(\alpha, v_{0}\right) \mid Q\right]=0$, by symmetry, because given $Q$, $T^{\prime}$ is just ordinary UST on $G^{W}\left(B\left(v_{0}, s / 2\right), \delta\right)$. But $\alpha$ is also a path in $T$. Let $\beta$ be the path in $T$ from $a$ to the endpoint of $\gamma_{\mathrm{r}}^{\delta}$ near $\partial B\left(v_{0}, r\right)$. Then $X_{0}=$ $W\left(\alpha, v_{0}\right)+W\left(\beta, v_{0}\right)+W_{\delta}(r)$, and therefore,

$$
\begin{aligned}
W_{\delta}(r)-\mathbf{E}\left[X_{0} \mid Q\right] & =-\mathbf{E}\left[W\left(\alpha, v_{0}\right) \mid Q\right]-\mathbf{E}\left[W\left(\beta, v_{0}\right) \mid Q\right] \\
& =-\mathbf{E}\left[W\left(\beta, v_{0}\right) \mid Q\right] .
\end{aligned}
$$

Let $t>\delta$. Note that if $s<t$, then there are at least two disjoint crossings in $T$ of the annulus $A\left(v_{0}, t, r / 10\right)$ (both on the path $Q_{v}^{0}, v \in V_{0}$, which has minimum distance to $v_{0}$ ). Therefore, Lemma 8.2 gives

$$
\mathbf{P}[s<t] \leqslant O(1)(t / r)^{C_{7}} .
$$

Fix some $y \geqslant 2$. By Lemma 8.3, we have

$$
\mathbf{P}\left[\left|W\left(\beta, v_{0}\right)\right|>t, s \geqslant r / y\right] \leqslant O(1) \exp \left(-C_{3} t / \log y\right) \log y .
$$

Hence, using (8.8),

$$
\begin{aligned}
\mathbf{P}\left[\left|W\left(\beta, v_{0}\right)\right|>t\right] & \leqslant \mathbf{P}[s<r / y]+O(1) \exp \left(-C_{3} t / \log y\right) \log y \\
& \leqslant O(1) y^{-C_{7}}+O(1) \exp \left(-C_{3} t / \log y\right) \log y .
\end{aligned}
$$

Assuming that $t \geqslant 1$, we may choose $y=\exp \sqrt{t}$, and then get

$$
\mathbf{P}\left[\left|W\left(\beta, v_{0}\right)\right|>t\right] \leqslant O(1) \sqrt{t} \exp \left(-C_{8} \sqrt{t}\right),
$$

for some constant $C_{8}>0$. This gives $\mathbf{E}\left[W\left(\beta, v_{0}\right)^{2}\right]=O(1)$. But for every random variable $Y$, we have $\mathbf{E}\left[Y^{2}\right] \geqslant \mathbf{E}\left[\mathbf{E}[Y \mid Q]^{2}\right]$. Therefore, (8.7) implies (8.6) for $j=0$. The proof of (8.6) for $j=1$ is entirely the same. This completes the proof of the proposition.

Proposition 8.6: Assuming Conjecture 1.2, $\kappa_{o}=2$, where $\kappa_{o}$ is the constant such that SLE with parameter $\kappa_{o}$ is the scaling limit of LERW.

Proof: Recall the definition of $W_{\delta}(r)$, which appears above Proposition 8.5. Set $r_{0}=1 / 2$, and let $r_{1}>0$ be very small. Let $\psi: \mathbb{H} \rightarrow \mathbb{U}$ be the conformal 
map satisfying $\psi(i)=0$ and $\psi^{\prime}(i)>0$. Let $C_{j}$ be the circle of radius $r_{j}$ about $i, j=0,1$, and set $C_{j}^{\prime}:=\psi\left(C_{j}\right)$. Note that $Z_{\delta}\left(r_{1}\right)=W_{\delta}\left(r_{1}\right)-W_{\delta}\left(r_{0}\right)$ is the winding number around $i$ of some arc on $\gamma^{\delta}$, the LERW from a vertex near $i$ to $\partial \mathbb{H}$ in $\delta \mathbb{Z}^{2} \cap \mathbb{H}$, and the arc has one endpoint near the circle $\partial B\left(i, r_{0}\right)$ and the other endpoint near the circle $\partial B\left(i, r_{1}\right)$. It follows that $Z_{\delta}\left(r_{1}\right)$ converges weakly to a winding number $Z\left(r_{1}\right)$ of an $\operatorname{arc} \beta$ of $\psi^{-1}\left(\xi_{\kappa_{o}}\right)$ with endpoints on $C_{0}$ and $C_{1}$, as $\delta \rightarrow 0$ along some sequence, where $\xi_{\kappa_{o}}$ is the SLE curve with parameter $\kappa_{o}$. Moreover, since we have good tail estimates on $Z_{\delta}\left(r_{1}\right)$ (Lemma 8.3), from the dominated convergence theorem it follows that

$$
\mathbf{E}\left[Z\left(r_{1}\right)^{2}\right]=\lim _{\delta \rightarrow 0} \mathbf{E}\left[Z_{\delta}\left(r_{1}\right)^{2}\right] .
$$

Hence, Proposition 8.5 gives $\mathbf{E}\left[Z\left(r_{1}\right)^{2}\right]=2 \log \left(1 / r_{1}\right)+O(1) \sqrt{\log \left(1 / r_{1}\right)}$. Observe that for any path $\alpha$ in $\mathbb{H}-\{i\}$, the winding number of $\alpha$ around $i$ minus the winding number of $\psi(\alpha)$ around 0 is bounded by some constant. Consequently, the winding number $W^{\prime}$ of $\beta^{\prime}:=\psi(\beta)$ around 0 also satisfies

$$
\mathbf{E}\left[W^{\prime 2}\right]=2 \log \left(1 / r_{1}\right)+O(1) \sqrt{\log \left(1 / r_{1}\right)}
$$

Set $t_{j}=\log r_{j}, j=0,1$, let $\tilde{\beta}$ be the $\operatorname{arc} \xi_{\kappa_{o}}(t):\left[t_{1}, t_{0}\right] \rightarrow \mathbb{U}$, and let $\tilde{W}$ be the winding number of $\tilde{\beta}$ around 0 . By Theorem 7.2 , with high probability, the log of the absolute value of the endpoints of $\tilde{\beta}$ is not far from the log of the absolute value of the endpoints of $\beta^{\prime}$. Therefore, it is easy to conclude with the help of Lemma 8.3 that

$$
\mathbf{E}\left[\left(\tilde{W}-W^{\prime}\right)^{2}\right]=O(1)
$$

We know from Theorem 7.2 again that

$$
\mathbf{E}\left[\tilde{W}^{2}\right]=\kappa_{o}\left|t_{1}\right|+O(1) \sqrt{\left|t_{1}\right|} .
$$

Combining this with (8.9) and (8.10) gives

$$
\left(2-\kappa_{o}\right)\left|t_{1}\right|=O(1) \sqrt{\left|t_{1}\right|} .
$$

Letting $t_{1} \rightarrow-\infty$ now completes the proof.

Proof of Theorem 1.3: Immediate from Corollary 6.2 and Proposition 8.7. 


\section{The critical value for the SLE}

Theorem 9.1: $\sup \mathfrak{K} \leqslant 4$, where $\mathfrak{K}$ is as in Definition 7.1.

Proof: Fix some $\kappa \in \mathfrak{K}$, and let $f_{t}$ be the solution of the Löwner equation with parameter $\zeta(t)=\mathrm{B}(-\kappa t)$, where $\mathrm{B}:[0, \infty) \rightarrow \partial \mathbb{U}$ is Brownian motion starting from a uniform point in $\partial \mathbb{U}$. Note the for every $t<0$ the map $f_{t}^{-1}$ is well defined and injective on $\partial \mathbb{U}-\{\zeta(0)\}$, since $f_{t}$ is a Riemann map onto a slit domain, and the slit hits $\partial \mathbb{U}$ at $\zeta(0)$. Set $b(t):=-i \log f_{t}^{-1}(1)$, with $b(0)-\widehat{\mathrm{B}}(0) \in[0,2 \pi)$ and $b(t)$ continuous in $t$. (A.s. $\zeta(0) \neq 1$, and on this event $b(t)$ is well defined for all $t<0$.) Then $b(t)$ is real. As in (7.5), we have

$$
b^{\prime}(t)=\frac{\sin (\widehat{\mathrm{B}}(-\kappa t)-b(t))}{1-\cos (\widehat{\mathrm{B}}(-\kappa t)-b(t))}=\cot \frac{1}{2}(\widehat{\mathrm{B}}(-\kappa t)-b(t)) .
$$

Let $p(s)=b(-s)-\widehat{\mathrm{B}}(\kappa s)$, and let $\epsilon>0$. Set $\tau_{\epsilon}=\inf \{s \geqslant 0: p(s)=\epsilon\}$ and $\tau_{\pi}=\inf \{s \geqslant 0: p(s)=\pi\}$. For $x \in[\epsilon, \pi]$, let $g_{\epsilon}(x)$ be the probability that $\tau_{\pi}<\tau_{\epsilon}$, conditioned on $p(0)=x$. Also set $g_{\epsilon}(x)=0$ for $x<\epsilon$ and $g_{\epsilon}(x)=1$ for $x>\pi$. We now show that $g_{\epsilon}$ satisfies

$$
\frac{\kappa}{2} g_{\epsilon}^{\prime \prime}(x)+g_{\epsilon}^{\prime}(x) \cot (x / 2)=0
$$

inside $(\epsilon, \pi)$, using Itô's formula. (The reader unfamiliar with stochastic calculus can have a look at [Dur84], for example, or try to derive (9.2) directly. The latter is a bit tricky, but can be done.) Observe that $g_{\epsilon}\left(p\left(s^{*}\right)\right)$ is a martingale, where $s^{*}=\min \left\{s, \tau_{\epsilon}, \tau_{\pi}\right\}$. By $(9.1)$, we have

$$
d p(s)=-b^{\prime}(-s) d s-d \widehat{\mathrm{B}}(\kappa s)=\cot (p(s) / 2) d s-d \widehat{\mathrm{B}}(\kappa s),
$$

and therefore, by Itô's Formula (assuming, for the moment, that $g_{\epsilon}$ is $C^{2}$ ),

$$
\begin{aligned}
d g_{\epsilon}(p(s)) & =g_{\epsilon}^{\prime}(p(s))(\cot (p(s) / 2) d s-d \widehat{\mathrm{B}}(\kappa s))+(1 / 2) g_{\epsilon}^{\prime \prime}(p(s)) d\langle\widehat{\mathrm{B}}(\kappa s)\rangle \\
& =\left(\cot (p(s) / 2) g_{\epsilon}^{\prime}(p(s))+(\kappa / 2) g_{\epsilon}^{\prime \prime}(p(s))\right) d s-g_{\epsilon}^{\prime}(p(s)) d \widehat{\mathrm{B}}(\kappa s)
\end{aligned}
$$

for $s<\min \left\{\tau_{\epsilon}, \tau_{\pi}\right\}$. Since $g_{\epsilon}\left(p\left(s^{*}\right)\right)$ is a martingale, the $d s$ term must vanish, and so (9.2) holds inside $(\epsilon, \pi)$. Consequently, in that range,

$$
g_{\epsilon}^{\prime}(x)=c_{\epsilon}(\sin (x / 2))^{-4 / \kappa},
$$

where $c_{\epsilon}$ is some constant depending on $\epsilon$. Since $g_{\epsilon}(\epsilon)=0$ and $g_{\epsilon}(\pi)=1$, we have $\int_{\epsilon}^{\pi} g_{\epsilon}^{\prime}(x) d x=1$, which gives

$$
c_{\epsilon}^{-1}=\int_{\epsilon}^{\pi}(\sin (x / 2))^{-4 / \kappa} d x .
$$


We know that a.s. $p(s) \neq 0$ for all $s$, which is equivalent to $\lim _{\epsilon \rightarrow 0} g_{\epsilon}(x)=1$ on $(0, \pi)$. This gives $\lim _{\epsilon \rightarrow 0} g_{\epsilon}^{\prime}(x)=0$, that is, $\lim _{\epsilon \rightarrow 0} c_{\epsilon}=0$. Therefore, $\kappa \leqslant 4$.

This completes the proof, except that we have not shown that $g_{\epsilon}$ is $C^{2}$ (there should be a reference implying this, but we have not located one). To deal with this, the above procedure is reversed. Define $g_{\epsilon}$ as the solution of (9.2) satisfying $g_{\epsilon}(\epsilon)=0$ and $g_{\epsilon}(1)=1$. Then the above application of Itô's Formula shows that $g_{\epsilon}\left(p\left(s^{*}\right)\right)$ is a martingale. By the Optional Sampling Theorem, this implies that $g_{\epsilon}(x)$ is the probability that $\tau_{\pi}<\tau_{\epsilon}$, conditioned on $p(0)=x$, and completes the proof.

Conjecture 9.2: $\mathfrak{K}=[0,4]$.

\section{Properties of UST subsequential scaling limits in two dimensions}

Before we go into the study of the UST scaling limit, let us remark that the definition we have adopted for the scaling limit is by no means the only reasonable one. There are several other reasonable variations, and choosing one is partly a matter of convenience and taste.

We now recall some definitions. Again, we think of $\delta \mathbb{Z}^{2}$ as a subset of the sphere $\mathbb{S}^{2}=\mathbb{R}^{2} \cup\{\infty\}$. Recall that $\hat{\mathrm{T}}_{\delta}$ denotes the UST on $\delta \mathbb{Z}^{2}$, with the point $\infty$ added, to make it compact. Given two points $a, b \in \widehat{T}_{\delta}, a \neq b, \omega_{a, b}=\omega_{a, b}^{\delta}$ denotes the unique path in $\hat{\mathrm{T}}_{\delta}$ with endpoints $a$ and $b$. For the case $a=b$, we set $\omega_{a, a}=\{a\}$. Let $\mathfrak{T}_{\delta}$ be the collection of all triplets, $\left(a, b, \omega_{a, b}\right)$, where $a, b \in \widehat{T}_{\delta}$. $\mathfrak{T}_{\delta}$ will be called the paths ensemble of $\hat{T}_{\delta}$. Let $\mathfrak{T}$ denote a random variable in $\mathcal{H}\left(\mathbb{S}^{2} \times \mathbb{S}^{2} \times \mathcal{H}\left(\mathbb{S}^{2}\right)\right)$ whose law is a weak subsequential limit of the law of $\mathfrak{T}_{\delta}$ as $\delta \rightarrow 0$. The trunk is defined by

$$
\text { trunk }=\operatorname{trunk}(\mathfrak{T}):=\bigcup_{(a, b, \omega) \in \mathfrak{T}}(\omega-\{a, b\}) .
$$

Let $\epsilon \in(0,1]$ and $a, b \in \widehat{\mathrm{T}}_{\delta}$. We define $\omega_{a, b}(\epsilon)$ as follows. Let $a^{\prime}$ be the first point along the path $\omega_{a, b}$ (which is oriented from $a$ to $b$ ) where $d\left(a, a^{\prime}\right)=\epsilon$, and let $b^{\prime}$ be the last point along the path where $d\left(b, b^{\prime}\right)=\epsilon$, provided that such points exist. If $a^{\prime}$ and $b^{\prime}$ exist, and $a^{\prime}$ appears on the path before $b^{\prime}$, then let $\omega_{a, b}(\epsilon)$ be the (closed) subarc of $\omega_{a, b}$ from $a^{\prime}$ to $b^{\prime}$; and otherwise set $\omega_{a, b}(\epsilon)=\emptyset$. Let $\mathfrak{T}_{\delta}(\epsilon)$ denote the set of all triplets $\left(a, b, \omega_{a, b}(\epsilon)\right)$ such that $a, b \in \hat{\mathrm{T}}_{\delta}$ and $\omega_{a, b}(\epsilon) \neq \emptyset$. Note that if $d(a, b)>2 \epsilon$, then $\omega_{a, b}(\epsilon) \neq \emptyset$. We define

$$
\operatorname{trunk}_{\delta}(\epsilon):=\bigcup\left\{\omega:(a, b, \omega) \in \mathfrak{T}_{\delta}(\epsilon)\right\}
$$


Then $\operatorname{trunk}_{\delta}(\epsilon)$ is a compact subset of $\widehat{\mathrm{T}}_{\delta}$, which we call the $\epsilon$-trunk of $\widehat{\mathrm{T}}_{\delta}$. By compactness, for every $\epsilon \in(0,1]$ there is a subsequential scaling limit of the law of trunk $k_{\delta}(\epsilon)$. By passing to a subsequence, if necessary, we assume that for all $n \in \mathbb{N}_{+}:=\{1,2, \ldots\}$ the weak limit trunk $0(1 / n)$ of $\operatorname{trunk}_{\delta}(1 / n)$, as $\delta \rightarrow 0$, exists.

Recall that the dual $T^{\dagger}$ of a spanning tree $T \subset \delta \mathbb{Z}^{2}$ is the spanning subgraph of the dual graph $\left(\delta \mathbb{Z}^{2}\right)^{\dagger}:=(\delta / 2, \delta / 2)+\delta \mathbb{Z}^{2}$ containing all edges that do not intersect edges in $T$. If $T$ is the UST on $\delta \mathbb{Z}^{2}$, then $T^{\dagger}$ has the law of the UST on $\left(\delta \mathbb{Z}^{2}\right)^{\dagger}$. (See, e.g., [BLPS98].) Let $\widehat{T}_{\delta}^{\dagger}$ be $T^{\dagger} \cup\{\infty\}$, where $T$ is the UST on $\delta \mathbb{Z}^{2}$. trunk $k^{\dagger}$ and trunk $k_{0}^{\dagger}(\epsilon)$ are defined for $\hat{T}_{\delta}^{\dagger}$ as trunk and trunk $k_{0}(\epsilon)$ were defined for $\widehat{T}_{\delta}$.

We may think of the random variables trunk, trunk ${ }^{\dagger}$, trunk $k_{0}(1 / n)$, and $\operatorname{trunk}_{0}^{\dagger}(1 / n)\left(n \in \mathbb{N}_{+}\right)$as defined on the same probability space, by taking a subsequential limit of the joint distribution of $\mathfrak{T}_{\delta}, \mathfrak{T}_{\delta}^{\dagger},\left\langle\operatorname{trunk}_{\delta}(1 / n): n \in \mathbb{N}_{+}\right\rangle$, and $\left\langle\operatorname{trunk}_{\delta}^{\dagger}(1 / n): n \in \mathbb{N}_{+}\right\rangle$. It is immediate to verify that a.s.

$$
\text { trunk }=\bigcup_{n \in \mathbb{N}_{+}} \text {trunk }_{0}(1 / n),
$$

and trunk ${ }_{0}(1 /(n+1)) \supset$ trunk $_{0}(1 / n)$ for $n \in \mathbb{N}_{+}$.

We shall prove that trunk is a.s. a topological tree, in the sense of the following definition.

Definition 10.1: Trees. An arc joining two points $x, y$ in a metric space $X$ is a set $J \subset X$ such that there is a homeomorphism $\phi:[0,1] \rightarrow J$ with $\phi(0)=x$ and $\phi(1)=y$. A metric space $X$ will be called a topological tree if it is uniquely arcwise connected (that is, given $x \neq y$ in $X$ there is a unique $\operatorname{arc}$ in $X$ joining $x$ and $y$ ) and locally arcwise connected (that is, whenever $x \in U$ and $U$ is an open subset of $X$ there is an open $W \subset U$ with $x \in W$ and $W$ is arcwise connected). A finite topological tree is a topological space which is homeomorphic to a finite, connected, simply connected, 1-dimensional simplicial complex.

Note that a connected subset of a topological tree is a topological tree [Bow]. Although we shall not need this fact, it is instructive to note that a metric space which is a topological tree is homeomorphic to an $\mathbb{R}$-tree ${ }^{4}$ [MO90] (see also [MMOT92], for a slightly less general but simpler proof).

The next theorem establishes a finiteness property of the $\epsilon$-trunks, which is the first step in the proof of Theorem 1.6.

4 An $\mathbb{R}$-tree is a metric space $(T, d)$ such that for every two distinct points $x, y \in T$ there is a unique isometry $\phi$ from $[0, d(x, y)]$ onto a subset of $T$ satisfying $\phi(0)=x$ and $\phi(d(x, y))=y$. 
THEOREM 10.2 (Finiteness): For every $\epsilon>0$ there is a $\widehat{\delta}>0$ with the following property. Suppose that $0<\delta<\widehat{\delta}$. Let $\widehat{V}$ be a set of vertices of $\delta \mathbb{Z}^{2}$ such that every point in $\mathbb{S}^{2}$ is within distance $\widehat{\delta}$ of some vertex in $\widehat{V}$. Let $Q:=Q_{\delta}(\widehat{V})$ be the subtree of $\widehat{T}_{\delta}$ that is spanned by $\widehat{V}$, that is, the minimal connected subset of $\widehat{\mathrm{T}}_{\delta}$ containing $\widehat{V}$. Then with probability at least $1-\epsilon$ we have $Q \supset \operatorname{trunk}_{\delta}(\epsilon)$.

Proof: Fix some small $\widehat{\delta}>0$, and suppose that $\delta \in(0, \widehat{\delta})$. Let $V_{0}:=\widehat{V}$, and for each $j \in \mathbb{N}_{+}$let $V_{j}$ be a set of vertices containing $V_{j-1}$ such that every vertex of $\delta \mathbb{Z}^{2}$ is within spherical distance $\delta_{j}:=2^{-j} \widehat{\delta}$ of some vertex in $V_{j}$, and $V_{j}$ is a minimal set satisfying these properties. Note that the number of vertices in $V_{j}-V_{j-1}$ is bounded by $O(1) \delta_{j}^{-2}$. Let $Q_{j}$ be the subtree of $\widehat{\mathrm{T}}_{\delta}$ spanned by $V_{j}$.

We now estimate the probability that there is some component of $Q_{j+1}-Q_{j}$ whose diameter is large. Let $v$ be some vertex in $\delta \mathbb{Z}^{2}$, let $Q(v, j)$ be the arc of $\widehat{\mathrm{T}}_{\delta}$ that connects $v$ to $Q_{j}$, and let $\mathcal{D}(v, j, a)$ be the event the diameter of $Q(v, j)$ is at least $a \delta_{j}$. By Wilson's algorithm, we may obtain $Q(v, j)$ by conditioning on $Q_{j}$ and loop-erasing a simple random walk from $v$ that stops when $Q_{j}$ is hit. Every vertex $w \in \delta \mathbb{Z}^{2}$ is within distance $\delta_{j}$ from a vertex in $Q_{j}$. Since $Q_{j}$ is connected and has diameter at least 1, Lemma 2.1 shows that there is a universal constant $C_{0}>0$ so that the probability that a random walk from $w$ gets to distance $C_{0} \delta_{j}$ from $w$ before hitting $Q_{j}$ is at most $1 / 2$. Consequently, $\mathcal{D}(v, j, a)$ has probability at most $O(1) \exp \left(-C_{1} a\right)$, where $C_{1}>0$ is an absolute constant. We choose $a_{j}:=j^{2}(\log \widehat{\delta})^{2} / C_{1}$. Since there are at most $O(1) \delta_{j}^{-2}$ vertices in $V_{j+1}-V_{j}$, we find that the probability of

$$
\mathcal{D}:=\bigcup_{j=1}^{\infty} \bigcup_{v \in V_{j+1}} \mathcal{D}\left(v, j, a_{j}\right)
$$

is bounded by

$$
O(1) \widehat{\delta}^{-2} \sum_{j=1}^{\infty} 2^{2 j} \exp \left(-j^{2}(\log \widehat{\delta})^{2}\right),
$$

which goes to zero as $\widehat{\delta} \rightarrow 0$.

Let $v \in \delta \mathbb{Z}^{2}$. There is a sequence $v_{1}, v_{2}, \ldots, v_{n}$ with $v_{j} \in V_{j}$ such that $Q(v, 1) \subset$ $\bigcup_{j=2}^{n} Q\left(v_{j}, j-1\right)$, and the latter union is connected. If we are in the complement of $\mathcal{D}$, it follows that the diameter of $Q(v, 1)$ is at most $s:=\sum_{j=1}^{\infty} a_{j} \delta_{j}$. Since $s \rightarrow 0$ as $\delta_{0} \rightarrow 0$, this establishes the theorem.

Several corollaries follow from this theorem. 
Corollary 10.3: For each $n \in \mathbb{N}_{+}$, a.s. trunk $k_{0}(1 / n)$ is a finite topological tree.

Proof: Let $W \subset \mathbb{R}^{2}$ be finite. For each $w \in W$ and $\delta>0$, let $w_{\delta} \in \delta \mathbb{Z}^{2}$ be closest to $w$, with ties broken arbitrarily, and set $W_{\delta}=\left\{w_{\delta}: w \in W\right\}$. Let $Q_{\delta}(W)$ be the subtree of $\hat{\mathrm{T}}_{\delta}$ spanned by $W_{\delta}$. The theorem shows that we may choose a finite $W \subset \mathbb{R}^{2}$ such that $\operatorname{trunk}_{\delta}(1 / n) \subset Q_{\delta}(W)$ with probability at least $1-\epsilon$, for every sufficiently small $\delta>0$. Consequently, we may couple a subsequential scaling limit $Q(W)$ of $Q_{\delta}(W)$ as $\delta \rightarrow 0$ so that trunk $(1 / n) \subset Q(W)$ with probability at least $1-\epsilon$. Because trunk $\mathrm{k}_{0}(1 / n)$ is connected and $\epsilon$ is an arbitrary positive number, it suffices to prove that $Q(W)$ is a.s. a finite tree. The latter is easily proved by induction on $|W|$ using Theorem 1.1, Wilson's algorithm, and the following easy fact: the tree spanned by a subset of the points in $W$ is unlikely to pass close by to the other points. (See Remark 3.2.)

Corollary 10.4: The Hausdorff dimension of trunk is in $(1,2)$. Moreover, if $I=\left(s_{0}, s_{1}\right]$ is an interval such that a.s. the Hausdorff dimension of any scaling limit of $L E R W$ is in $I$, then the Hausdorff dimension of trunk(T) is in $I$.

Proof: The second statement follows immediately from Theorem 10.2. The first is now a consequence of the result of [ABNW], showing that there are $s_{0}, s_{1} \in$ $(1,2)$ such that a.s. the Hausdorff dimension of LERW scaling limit is in $\left[s_{0}, s_{1}\right] .^{5}$

Remark 10.5: The above-mentioned lower bound in [ABNW] is based on the ideas of [BJPP97]. Kenyon [Ken] can prove that we may take $s_{1}=5 / 4$. In earlier work [Ken98b] he showed that $n^{5 / 4}$ times the expected number of edges in a LERW from $(0,0)$ to the boundary of the square $[-n, n]^{2}$ tends to a finite positive constant as $n \rightarrow \infty$. This supports the conjecture that the Hausdorff dimension of the scaling limit of LERW is a.s. $5 / 4$, and the same would apply to $\operatorname{trunk}(\mathfrak{T})$.

The degree of a point $p$ in a topological tree $T$ is the number of connected components of $T-\{p\}$. The following corollary is a strong form of the statement that the maximum degree of points in trunk $k_{0}(1 / n)$ is 3 . From this and the fact that trunk is a tree (which we prove further below) it immediately follows that the

5 From Remark 3.2 follows the weaker result that the area measure of any subsequential scaling limit of LERW is zero, hence that the area of trunk is zero. It is likely that with a bit more effort the proof of Remark 3.2 is sufficient for the stronger claim that the Hausdorff dimension is smaller than 2. 
maximum degree in trunk is 3 , because every finite subset of trunk is contained in some trunk $0(1 / n)$.

Given a point $p \in \mathbb{S}^{2}$ and two numbers $0<r_{1}<r_{2}<1$, let $A_{\mathrm{sp}}\left(p, r_{1}, r_{2}\right)$ denote the annulus with center $p$, inner radius $r_{1}$, and outer radius $r_{2}$, in the spherical metric.

Corollary 10.6: Given every $\epsilon \in(0,1)$, there is an $r \in(0, \epsilon)$ with the following property. For every sufficiently small $\delta>0$, the probability that there is a point $p \in \mathbb{S}^{2}$ such that there are 4 disjoint crossings in $\operatorname{trunk}_{\delta}(\epsilon)$ of the annulus $A_{\mathrm{sp}}(p, r, \epsilon)$ is at most $\epsilon$.

By having 4 disjoint crossings in $\operatorname{trunk}_{\delta}(\epsilon)$ of an annulus $A$, we mean that there are 4 disjoint connected subsets of $\operatorname{trunk}_{\delta}(\epsilon) \cap A$ that intersect both boundary components of $A$. Below, Corollary 10.11 gives a strengthening of Corollary 10.16 .

Proof: By Theorem 10.2, it is enough to prove the statement with $Q_{\delta}(W)$ replacing $\operatorname{trunk}_{\delta}(\epsilon)$, where $W \subset \mathbb{R}^{2}$ is a set of bounded size, provided that the value of $r$ does not depend on $\delta$. Again, induction on $|W|$ can be used together with Wilson's algorithm. One needs note the following easy facts. The tree $T_{k-1}$ spanned by $k-1$ points of $W$ is unlikely to pass close to the other points of $W$, and when adding a further point, it is unlikely that the attachment point of the new branch on $T_{k-1}$ will be close to another branch point. Also, once a random walk from the new point gets close to $T_{k-1}$ it will hit $T_{k-1}$ close by, with high likelihood. The easy details are left to the reader.

We now turn to the central issue in the proof of Theorem 1.6, which is,

THEOREM 10.7: In any subsequential scaling limit of UST in $\mathbb{S}^{2}$, a.s. the trunk and dual trunk do not intersect.

LEMMA 10.8: Given $\delta, \epsilon>0$, let $T_{\delta}^{3}(\epsilon)$ be the set of points of degree 3 in trunk $\delta(\epsilon)$. Let trunk ${ }_{\delta}^{\dagger}(\epsilon)$ be the $\epsilon$-trunk of the dual tree $\hat{\mathrm{T}}_{\delta}^{\dagger}$. Let $D$ be the spherical distance from $T_{\delta}^{3}(\epsilon)$ to $\operatorname{trunk}_{\delta}^{\dagger}(\epsilon)$, that is, the least spherical distance between a point in $T_{\delta}^{3}(\epsilon)$ to a point in $\operatorname{trunk}_{\delta}^{\dagger}(\epsilon)$. Then $\lim _{t \rightarrow 0} \mathbf{P}[D<t] \rightarrow 0$ uniformly in $\delta$.

The following simple observation is used in the proof. Suppose that we condition on a set of edges $S$ to appear in the UST tree in a planar graph. For the dual tree, this is the same as deleting the edges dual to the edges in $S$. Consequently, one can perform a variation on Wilson's algorithm for a planar graph, where one switches back and forth from building the tree by adding LERW branches 
and building the dual tree. When building the tree, the LERW acts with the constructed tree as a wired absorbing boundary and the constructed dual tree as a free boundary, and conversely when building the dual tree.

Proof: We first choose a large but finite collection of points $Q$ in $\delta \mathbb{Z}^{2}$ so that with high probability the subtree $T$ of $\widehat{\mathrm{T}}_{\delta}$ spanned by $Q$ contains $\operatorname{trunk}_{\delta}(\epsilon)$ (and $|Q|$ does not depend on $\delta$ ). This can be done, by Theorem 10.2. Let $Q^{\dagger}$ be a set of vertices of the dual graph $\left(\delta \mathbb{Z}^{2}\right)^{\dagger}$, such that with high probability the subtree $T^{\dagger}$ spanned by $Q^{\dagger}$ in the dual graph contains trunk $k_{\delta}^{\dagger}(\epsilon)$. Let $a_{1}, a_{2}, a_{3} \in Q$ and $a_{1}^{\dagger}, a_{2}^{\dagger} \in Q^{\dagger}$ be distinct points. It suffices to show that the probability that the arc $\beta$ joining $a_{1}^{\dagger}$ and $a_{2}^{\dagger}$ in $T^{\dagger}$ comes within distance $t$ of the meeting point $m$ of $a_{1}, a_{2}$ and $a_{3}$ in $T$ goes to zero as $t \rightarrow 0$, uniformly in $\delta$. This is easy. We condition on the subtree $T_{0}$ of $T$ spanned by $a_{1}, a_{2}, a_{3}$. Let $z^{\dagger}$ be a dual vertex close to $a_{1}^{\dagger}$. Then with high probability the dual tree path $\beta$ from $z^{\dagger}$ to $a_{1}^{\dagger}$ has diameter not much larger than the distance from $z^{\dagger}$ to $a_{1}^{\dagger}$. In particular, it does not go close to $T_{0}$. By the next lemma, conditioned on $\beta$ and $T_{0}$, the probability that a simple random walk starting at $a_{2}^{\dagger}$, with $T_{0}$ acting as a reflecting boundary, will get to within distance $t$ of $m$ before hitting $\beta$ tends to zero with $t$. Consequently, the same is true for the loop-erasure of this walk, which can be taken as the path joining $\beta$ and $a_{2}^{\dagger}$ in the dual tree.

A simple random walk on $\delta \mathbb{Z}^{2}$ is likely to hit a connected subgraph of fixed diameter $>0$ before visiting a specified vertex at fixed positive distance away. The next lemma gives a uniform version of this statement, in a slightly more general setting, where there is also a reflecting boundary.

Lemma 10.9: Let $D$ be a domain in $\mathbb{S}^{2}$ such that $\mathbb{S}^{2}-D$ has two connected components, $B_{1}, B_{2}$, and assume that neither is a single point. Consider a sequence $\delta_{j}, j \in \mathbb{N}$, of positive numbers tending to zero. Suppose that to each $j \in \mathbb{N}$ there are two connected subgraphs $B_{1}^{j}, B_{2}^{j}$ of the grid $\delta_{j} \mathbb{Z}^{2}$, and that $B_{1}^{j} \rightarrow B_{1}$ and $B_{2}^{j} \rightarrow B_{2}$ in the Hausdorff metric on compact subsets of $\mathbb{S}^{2}$. Let $m \in B_{2}$ be some point, and for each $t>0$ and $z \in \delta_{j} \mathbb{Z}^{2}-B_{2}^{j}$, let $h_{j}(z, t)$ be the probability that simple random walk on $\delta_{j} \mathbb{Z}^{2}-B_{2}^{j}$ starting at $z$ (with reflecting boundary conditions on $B_{2}^{j}$ ) will get to within distance $t$ of $m$ before hitting $B_{1}^{j}$. Let $K \subset \mathbb{S}^{2}-B_{2}$ be compact. Then

$$
\lim _{\substack{t \rightarrow 0 \\ j \rightarrow \infty}} \sup \left\{h_{j}(z, t): z \in K \cap \delta_{j} \mathbb{Z}^{2}\right\}=0 .
$$

Proof: Set $G_{j}:=\delta_{j} \mathbb{Z}^{2}-B_{2}^{j}$, let $S(t)$ be the vertices of $G_{j}$ that are within distance $t$ from $m$, and let $V_{j}(t)$ be the set of vertices of $G_{j}-B_{1}^{j}-S(t)$. Then 
$h_{j}(z, t)$ is discrete-harmonic in $V_{j}(t)$. Recall that the Dirichlet energy of $h_{j}(z, t)$ is $\sum\left(h_{j}(z, t)-h_{j}\left(z^{\prime}, t\right)\right)^{2}$, with the sum extending over all edges $\left[z, z^{\prime}\right]$ in $G_{j}$. Let $\lambda=\lambda_{j}$ be the minimum of $h_{j}(z, t)$ on $K$, and let $z$ be where the minimum is achieved. Then there is a path $\beta$ from $z$ to $S(t)$ such that $h_{j}(z, t) \geqslant \lambda$ on $\beta$, by the maximum principle for discrete harmonic functions. Note that one can find a collection of at least $1 /\left(c \delta_{j}\right)$ disjoint paths in $G_{j}$ which join $B_{1}^{j}$ and $\beta$ and each path in the collection has combinatorial length bounded by $c / \delta_{j}$, where $c<\infty$ depends only on $K$ and $D$. The Dirichlet energy of $h_{j}(z, t)$ restricted to each such path is at least $\lambda / \delta_{j}$ times a positive constant, and therefore the Dirichlet energy of $h_{j}(z, t)$ is at least $C \lambda$, where $C>0$ is a constant depending only on $K$ and $D$.

Let $d_{j}$ be the distance from $m$ to $B_{1}^{j}$. Since $h_{j}(z, t)$ is harmonic in $V_{j}(t)$, it minimizes the Dirichlet energy among functions on $G_{j}$ that are 1 on $S(t)$ and 0 on $B_{1}^{j}$. Therefore, the Dirichlet energy of $h_{j}(z, t)$ is at most the Dirichlet energy of the function $f: G_{j} \rightarrow \mathbb{R}$, which is 1 on $S(t), 0$ outside of $S\left(d_{j}\right)$, and equal to $\log \left(d_{j} /|z-m|\right) / \log \left(d_{j} / t\right)$ elsewhere, which is $O(1) / \log \left(d_{j} / t\right)$, as $j \rightarrow \infty$. This gives, $\lambda_{j}=O(1) / \log \left(d_{j} / t\right)$, and the lemma follows.

Proof of Theorem 10.7: Before we go into the actual details, the overall plan of the proof will be given (in a somewhat imprecise manner). Let $t_{0}>0$. It is not hard to reduce the theorem to the claim that with probability close to 1 the path $\gamma \subset \widehat{\mathrm{T}}_{\delta}$ which joins two fixed points $a_{1}, a_{2}$ does not have points $p$ close to it such that the path $\alpha_{p} \subset \widehat{T}_{\delta}$ joining $p$ to $\gamma$ is not contained in a small neighborhood of $\gamma$. Let $Z$ be the set of points $p$ such that $\alpha_{p}$ does not stay close to $\gamma$. When we condition on $\gamma$, the probability that $p \in Z$ goes to zero as $p$ tends to a point in $\gamma$, by a simple harmonic measure estimate. However, this is not enough, since there are many different $p$ 's close to $\gamma$. We fix some collection $L_{1}$ of points close to $\gamma$, and take a thick collection of points $L_{2}$ which are much closer to $\gamma$. What we show is that conditioned on $\gamma$ and on $Z \cap L_{2} \neq \emptyset$, the expectation of $N:=\left|Z \cap L_{1}\right|$ is much larger than $\mathbf{E}[N \mid \gamma]$. This is established by observing that when $p \in L_{1} \cap Z$ is appropriately chosen, the expected number of points $p^{\prime} \in L_{2}$ such that $\alpha_{p^{\prime}}$ is contained in $\alpha_{p}$, except for a small initial segment of $\alpha_{p^{\prime}}$, is quite large. It follows that

$$
\mathbf{P}\left[Z \cap L_{2} \neq \emptyset \mid \gamma\right] \leqslant \frac{\mathbf{E}[N \mid \gamma]}{\mathbf{E}\left[N \mid \gamma, Z \cap L_{1} \neq \emptyset\right]}
$$

is small, which suffices to prove the theorem.

We now give the details. Fix four distinct points $a_{1}, a_{2}, b_{1}, b_{2} \in \mathbb{R}^{2}$. Given $\delta>0$, let $a_{1}^{\prime}$ and $a_{2}^{\prime}$ be points of $\delta \mathbb{Z}^{2}$ that are closest to $a_{1}$ and $a_{2}$, respectively, 
and let $b_{1}^{\prime}$ and $b_{2}^{\prime}$ be vertices of the grid dual to $\delta \mathbb{Z}^{2}$ that are closest to $b_{1}$ and $b_{2}$, respectively. Let $\gamma$ be the path in $\widehat{\mathrm{T}}_{\delta}$ that joins $a_{1}^{\prime}$ and $a_{2}^{\prime}$, and given any $p \in \delta \mathbb{Z}^{2}$, let $\alpha_{p}$ denote the path in $\hat{\mathrm{T}}_{\delta}$ from $p$ to $\gamma$. Let $\beta$ be the path of $\hat{\mathrm{T}}_{\delta}^{\dagger}$ that joins $b_{1}^{\prime}$ and $b_{2}^{\prime}$.

Since trunk $=\bigcup_{n} \operatorname{trunk}_{0}(1 / n)$, and trunk ${ }^{\dagger}=\bigcup_{n} \operatorname{trunk}_{0}^{\dagger}(1 / n)$, Theorem 10.2 shows that it suffices to prove that the probability that the distance between $\gamma$ and $\beta$ is less than $t$ goes to zero, as $t$ goes down to zero, uniformly in $\delta$. We know that with probability close to one, $\beta$ does not come close to $\left\{a_{1}, a_{2}\right\}$, and $\gamma$ does not come close to $\left\{b_{1}, b_{2}\right\}$ (Remark 3.2). Therefore, we need only consider the situation where there is a point $q$ on $\gamma$, which is close to $\beta$, but not close to $\left\{a_{1}, a_{2}, b_{1}, b_{2}\right\}$. Since $\beta$ and $\gamma$ cannot cross, and since $\beta$ locally separates the sphere near every point of $\beta-\left\{b_{1}^{\prime}, b_{2}^{\prime}\right\}$, such a situation implies that there is a point $q^{\prime}$ in $\delta \mathbb{Z}^{2}$, which is near $q$, but in order to get to $\gamma$ from $q^{\prime}$ one must either cross $\beta$, or go "around" it. Consequently, diam $\left(\alpha_{q^{\prime}}\right)$ must be bounded away from zero, as $\alpha_{q^{\prime}}$ cannot cross $\beta$. It therefore suffices to rule out the existence of a point $q^{\prime} \in \delta \mathbb{Z}^{2}$ close to $\gamma$ but with diam $\left(\alpha_{q^{\prime}}\right)$ bounded away from zero. More precisely, let $K$ be a compact set disjoint from $\left\{a_{1}, a_{2}\right\}$, and let $\epsilon_{1} \in(0,1)$. Let $\tilde{h}$ be the least distance from $\gamma$ to some point $q^{\prime} \in K \cap \delta \mathbb{Z}^{2}$ such that diam $\left(\alpha_{q^{\prime}}\right) \geqslant \epsilon_{1}$. It suffices to show that

$$
\inf _{h_{0}>0} \limsup _{\delta \rightarrow 0} \mathbf{P}\left[\tilde{h}<h_{0}\right]=0 .
$$

Given any $p \in \delta \mathbb{Z}^{2}$, let $h(p)$ be the distance from $p$ to $\gamma$ and let $k(p)$ be the maximal distance from a point on $\alpha_{p}$ to $\gamma$. By Corollary 10.6, the probability that there is an $\operatorname{arc} \alpha$ in $\widehat{\mathrm{T}}_{\delta}$, which is disjoint from $\gamma$, satisfies $\operatorname{diam}(\alpha) \geqslant \epsilon_{1}$, and every point of $\alpha$ is within distance $t$ of $\gamma$, goes to zero as $t \rightarrow 0$, uniformly in $\delta$. Hence, to prove (10.2), it suffices to establish that

$$
\forall t>0 \quad \inf _{h_{0}>0} \limsup _{\delta \rightarrow 0} \mathrm{P}\left[\exists p \in K \cap \delta \mathbb{Z}^{2} h(p)<h_{0}, k(p) \geqslant t\right]=0 .
$$

Since the proof is somewhat involved, we consider first the simpler situation in which

$$
\gamma=[0,1] \times\{0\}, \text { and } K=[1 / 3,2 / 3] \times[0,1]
$$

(notwithstanding that this is an unrealistic situation, of extremely low probability). Obviously, it suffices to prove (10.3) for small $t>0$.

In the following arguments, several small positive quantities appear. Their dependence differs from the natural flow of the proof. In order to make it clear 
that the proof is logically sound, we state now that the dependence order is as follows:

$$
\epsilon_{0}, t_{0}, r_{0}, t_{1}, h_{1}, r_{1}, h_{2}, \delta
$$

that is, each of these quantities may depend only on those appearing before it in the list, and should be thought of as much smaller than its predecessors.

Set $h_{1}^{\prime}:=\max \left\{k \delta: k \in \mathbb{Z}, k \delta \leqslant h_{1}\right\}$, and let

$$
L_{1}:=\left\{\left(k \delta, h_{1}^{\prime}\right): k \in \mathbb{Z}, 1 / 6<k \delta<5 / 6\right\} .
$$

Given $\gamma$ and $p \in \delta \mathbb{Z}^{2}$, we may choose $\alpha_{p}$ by loop-erasing a simple random walk from $p$ to $\gamma$. Consequently, an easy harmonic measure estimate shows that $\mathbf{P}[k(p)>t]=O\left(h_{1} / t\right)$ for all $p \in L_{1}$.

Set $h_{2}^{\prime}:=\max \left\{k \delta: k \in \mathbb{Z}, k \delta \leqslant h_{2}\right\}$, and

$$
L_{2}:=\left\{\left(k \delta, h_{2}^{\prime}\right): k \in \mathbb{Z}, 1 / 4<k \delta<3 / 4\right\} .
$$

Again, for all $p \in L_{2}, \mathbf{P}\left[k(p) \geqslant t_{0}\right]=O\left(h_{2} / t_{0}\right)$, so we may assume that the event $\mathcal{Q}$ that the leftmost point in $L_{2}$ satisfies $k(p)<t_{0}$ has probability at least $1-\epsilon_{0}$. Let $\mathcal{K}$ be the event that there is some $p \in L_{2}$ with $k(p) \geqslant t_{0}$, and let $\mathcal{K}^{\prime}:=\mathcal{K} \cap \mathcal{Q}$. For proving (10.3) in the simpler situation (10.4), it suffices to show that $\mathbf{P}\left[\mathcal{K}^{\prime}\right]=O\left(\epsilon_{0}\right)$ for all sufficiently small $\delta>0$.

Consider the following procedure for generating $\hat{\mathrm{T}}_{\delta}$ given $\gamma$. Perform Wilson's algorithm starting with the vertices in $L_{2}$, in left-to-right order. If we encounter in this procedure some vertex $p \in L_{2}$ such that $k(p) \geqslant t_{0}$, we stop, and let $p_{0}$ denote that vertex. Let $T_{0}$ be the tree constructed up to that point (including $\left.\alpha_{p_{0}}\right)$. On the event $\mathcal{K}^{\prime}$, let $p_{1}$ be the first point on $\alpha_{p_{0}}$ whose distance to $\gamma$ is at least $t_{0}$, and let $\alpha$ be the arc of $\alpha_{p_{0}}$ from $p_{0}$ to $p_{1}$. Let $\mathcal{A}_{1}$ be the event that $\alpha$ is not contained in the rectangle $[1 / 5,4 / 5] \times\left[0, t_{0}\right]$. Note that $\mathcal{A}_{1}$ implies that there is an arc in $\alpha \cap\left([1 / 5,4 / 5] \times\left[0, t_{0}\right]\right)$ with diameter at least $1 / 15$. By considering this arc and $\gamma$, Corollary 10.6 shows that $\mathbf{P}\left[\mathcal{A}_{1} \cap \mathcal{K}^{\prime}\right]<\epsilon_{0}$, assuming that $t_{0}$ is sufficiently small.

On the event $\mathcal{K}^{\prime}-\mathcal{A}_{1}$, let

$$
x_{1}:=\max \left\{x \in \mathbb{R}:\left(x, h_{1} / 2\right) \in \alpha\right\},
$$

let $U$ be the component of $\mathbb{R}^{2}-\left(\alpha \cup\left(\left[x_{1}, \infty\right) \times\left\{h_{1} / 2\right\}\right) \cup\left(\mathbb{R} \times\left\{t_{1}\right\}\right)\right)$ that contains $\left(x_{1}, \infty\right) \times\left\{h_{1} / 2\right\}$, and let $U^{\prime}$ be the set of points in $U$ that are within distance $t_{1}$ from $\alpha$. See Figure 10.1. Let $\mathcal{A}_{2}$ be the event that $\mathcal{K}^{\prime}-\mathcal{A}_{1}$ occurs and $U^{\prime}$ intersects $T_{0}$. 


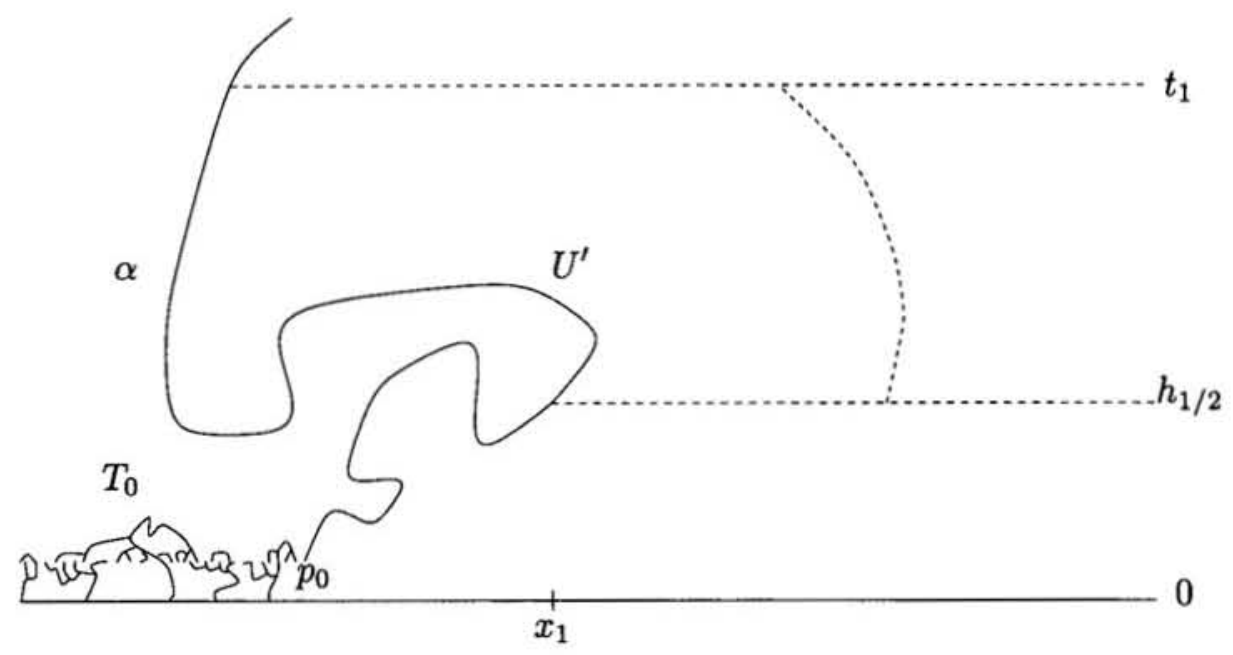

Figure 10.1.

We now prove that $\mathbf{P}\left[\mathcal{K}-\mathcal{A}_{1}-\mathcal{A}_{2}\right]=O\left(\epsilon_{0}\right)$. Let $N$ be the number of points $p \in L_{1}$ such that $k(p) \geqslant t_{0}$. For a given $p \in L_{1}$, the probability of $k(p) \geqslant t_{0}$ (given (10.4), but otherwise unconditioned) is $O\left(h_{1} / t_{0}\right)$. Therefore,

$$
\mathbf{E}[N] \leqslant O(1) h_{1} /\left(\delta t_{0}\right) .
$$

On the other hand, condition on the event $\mathcal{K}^{\prime}-\mathcal{A}_{1}-\mathcal{A}_{2}$ and on $T_{0}$. Let $L_{1}^{\prime}$ be the set of $p \in L_{1} \cap U$ such that the distance from $p$ to $\alpha$ is at most $t_{1} / 2$. Note that conditioned on $p \in L_{1}^{\prime}$, the probability that $\alpha_{p}$ joins with $\alpha_{p_{0}}$ within distance $2 t_{1}$ from $p$ is at least

$$
O(1)^{-1} \frac{h_{1}}{\operatorname{dist}(p, \alpha)+h_{1}},
$$

since after generating $T_{0}$, we may continue by running Wilson's algorithm starting at $p$, and the probability that the random walk starting at $p$ will hit $\alpha$ before the ray $\left(x_{1}, \infty\right) \times\left\{h_{1} / 2\right\}$ is at least (10.6). It therefore follows that conditioned on $\mathcal{K}-\mathcal{A}_{1}-\mathcal{A}_{2}$, we have

$$
\begin{aligned}
\mathbf{E}\left[N \mid \mathcal{K}-\mathcal{A}_{1}-\mathcal{A}_{2}\right] & \geqslant O(1)^{-1} \sum\left\{h_{1} /(k \delta): k \in \mathbb{Z}, h_{1} \leqslant k \delta \leqslant t_{1} / 2\right\} \\
& \geqslant O(1)^{-1} \delta^{-1} h_{1} \log \left(t_{1} /\left(2 h_{1}\right)\right) .
\end{aligned}
$$

Combining this with (10.5) gives

$$
\mathbf{P}\left[\mathcal{K}-\mathcal{A}_{1}-\mathcal{A}_{2}\right] \leqslant \frac{\mathbf{E}[N]}{\mathbf{E}\left[N \mid \mathcal{K}-\mathcal{A}_{1}-\mathcal{A}_{2}\right]} \leqslant O(1)\left(t_{0} \log \left(t_{1} /\left(2 h_{1}\right)\right)\right)^{-1} \leqslant \epsilon_{0},
$$


provided that $h_{1}$ is sufficiently small.

It remains to establish that $\mathbf{P}\left[\mathcal{A}_{2}\right] \leqslant O\left(\epsilon_{0}\right)$. First consider the case that there is some $p \in L_{2}$, to the left of $p_{0}$, such that $\alpha_{p} \cap U \neq \emptyset$. Then this must be the case for $p$ being the left neighbor of $p_{0}$, namely $p=p_{0}^{\prime}:=p_{0}-(\delta, 0)$, because when $p \in L_{2}$ is to the left of $p_{0}^{\prime}$, the path $\alpha_{p}$ cannot cross $\alpha_{p_{0}} \cup\left[p_{0}, p_{0}^{\prime}\right] \cup \alpha_{p_{0}^{\prime}}$, and $\alpha_{p}$ does not get to $\mathbb{R} \times\left\{t_{0}\right\}$. If $\alpha_{p_{0}^{\prime}}$ intersects $U$, then $\alpha_{p_{0}^{\prime}}$ must first get to some point $z$ in $\left[x_{1}, \infty\right) \times\left\{h_{1} / 2\right\}$. Near $z$ there must be two points $z_{1}^{\dagger}, z_{2}^{\dagger}$, which are vertices of the dual grid $\left(\delta \mathbb{Z}^{2}\right)^{\dagger}$, and are locally separated from each other by $\alpha_{p_{0}}$. The path in the dual tree that joins $z_{1}^{\dagger}$ and $z_{2}^{\dagger}$ has to contain the edge dual to the edge $\left[p_{0}^{\prime}, p_{0}\right]$. Now consider another dual vertex $z_{3}^{\dagger}$ just left of $\alpha$ near the point $\left(x_{1}, h_{1} / 2\right)$. Let $m^{\dagger}$ be the meeting point of $z_{1}^{\dagger}, z_{2}^{\dagger}$ and $z_{3}^{\dagger}$ in the dual tree. If $m^{\dagger}$ is not within distance $r_{1}$ of $p_{0}$, we get in the $r_{1} / 10$ trunk of the dual tree at least four disjoint crossings of the annulus $A\left(p_{0}, 2 h_{2}, r_{1} / 2\right)$. We may assume that this has probability $\leqslant \epsilon_{0}$, by Corollary 10.6. Similarly, Lemma 10.8, with the role of the tree and dual tree reversed, shows that we may take the event that $m^{\dagger}$ is within distance $r_{1}$ of $p_{0}$ to have probability $\leqslant \epsilon_{0}$, provided that $r_{1}$ is sufficiently small when compared with $h_{1}$.

To establish that $\mathbf{P}\left[\mathcal{A}_{2}\right] \leqslant O\left(\epsilon_{0}\right)$, it now suffices to prove that on the event $\mathcal{K}^{\prime}-\mathcal{A}_{1}$, the probability that $\alpha_{p_{0}}$ intersects $U^{\prime}$ is $O\left(\epsilon_{0}\right)$. The argument is similar here, but occurs on a larger scale. Suppose that $w \in \alpha_{p_{0}} \cap U^{\prime}$. Then $w$ must be on the segment of $\alpha_{p_{0}}$ from $p_{1}$ to the point $p_{2}$ in $\alpha_{p_{0}} \cap \gamma$. Because $w \in \alpha_{p_{0}}-\alpha$ and $w$ is within distance $t_{1}$ to $\alpha$, there is a point near $w$ that is in the $\left(t_{0} / 2\right)$-trunk of the dual tree, namely, some point on the path connecting dual two vertices on opposite sides of $\alpha_{p_{0}}$ near $p_{1}$. Consequently, by Lemma 10.8 , we may rule out the possibility that $p_{2}$ is within distance $r_{0}$ of $w$ as having small probability, since $p_{2}$ is a point of degree 3 of the $\left(t_{0} / 2\right)$-trunk. But if the distance between $p_{2}$ and $w$ is more than $r_{0}$, then there are in the $\left(t_{0} / 2\right)$-trunk at least four disjoint crossings of the annulus $A\left(w, 2 t_{1}, r_{0}\right)$ : two on $\alpha_{p_{0}}$ and two on $\gamma$. An appeal to Corollary 10.6 now establishes $\mathrm{P}\left[\mathcal{A}_{2}\right] \leqslant O\left(\epsilon_{0}\right)$. This completes the proof in the situation (10.4).

We now explain how to modify the above proof to deal with the general case. First note that the restriction on $K$ is entirely inconsequential; we could in the same way deal with any compact set disjoint from the endpoints of $\gamma$. More significant is the special selection of $\gamma$. Observe that under the assumption of conformal invariance of the LERW scaling limit, the general case can be reduced to the case where $\gamma=[0,1] \times\{0\}$, because after $\gamma$ is generated, the rest of the UST is just unconditioned UST on the complement of $\gamma$ with wired boundary 
conditions. We may then transform $\gamma$ by a conformal homeomorphism to $[0,1] \times\{0\}$, and refer to the above result.

Although we do not assume conformal invariance of the scaling limit, it turns out that the proof above is itself conformally invariant. With some care, one can apply the conformal map to the proof, in a manner of speaking. This is actually not very surprising, because the proof is ultimately based on a simple (discrete) harmonic measure estimate, which is conformally invariant.

Let us turn to the details. We may couple the UST for a subsequence of $\delta$ tending to zero so that $\gamma$ tends to some path $\gamma_{0}$ as $\delta \rightarrow 0$ along that subsequence (see the discussion of the Prohorov metric in Section 2). Let

$$
f_{\delta}: \mathbb{S}^{2}-([0,1] \times\{0\}) \rightarrow \mathbb{S}^{2}-\gamma
$$

be the conformal map normalized to take the endpoints of $[0,1] \times\{0\}$ to the endpoints of $\gamma$ and so that $f_{\delta}(\infty)$ is on the line which is the set of points at equal distance from both endpoints of $\gamma$, say. (The latter normalization is necessary to make $f_{\delta}$ unique, but otherwise, it is quite arbitrary.) It follows that $f_{\delta}$ tends to the similarly normalized conformal map $f: \mathbb{S}^{2}-([0,1] \times\{0\}) \rightarrow \mathbb{S}^{2}-\gamma_{0}$. We may assume that $\delta$ is so small that $f$ and $f_{\delta}$ are very close on compact subsets disjoint from $[0,1] \times\{0\}$.

For each $p \in L_{1}$, where $L_{1}$ is as before, we let $\widehat{p}$ denote a point in $\delta \mathbb{Z}^{2}$ that is closest to $f(p)$. For the general case, we consider $\widehat{N}$, the number of $p \in L_{1}$ such that $k(\widehat{p})$ is not small, in place of $N$. Let $\widehat{L}_{2}$ denote the set of points in $\delta \mathbb{Z}^{2}$ that are within distance $3 \delta$ of $f\left([1 / 4,3 / 4] \times\left\{h_{2}\right\}\right)$. The proof for the general case uses $\widehat{L}_{2}$ in place of $L_{2}$. For traversing $\widehat{L}_{1}$, there is no clear notion of the left-right order. But any ordering that starts near $f\left(\left(1 / 4, h_{2}\right)\right)$, and later does not visit any vertex before visiting an immediate neighbor, will do. Instead of the left neighbor $p_{0}^{\prime}$ of a vertex $p_{0} \in L_{1}$, we use for $\widehat{p}_{0} \in \widehat{L}_{1}$ that neighbor of $\widehat{p}_{0}$ in $\widehat{L}_{1}$ that is "most counterclockwise", in the appropriate sense. The rest of the proof proceeds with essentially no modifications, except that the coordinate system used is transformed by $f$.

Remark 10.10: In [BLPS98] it has been asked whether the free USF on every planar proper bounded degree graph is a tree. The proof of Theorem 10.7 seems to be relevant. It is plausible that with a similar argument one can prove that for proper planar graphs with bounded degree and a bounded number of sides per face, the free USF is a tree.

We may now strengthen Corollary 10.6, as follows 
Corollary 10.11: Given every $\epsilon \in(0,1)$, there is an $r \in(0, \epsilon)$ with the following property. For every sufficiently small $\delta>0$, the probability that there is a point $p \in \mathbb{S}^{2}$ such that there are 4 disjoint crossings in $\widehat{\mathrm{T}}_{\delta}$ of the annulus $A_{\mathrm{sp}}(p, r, \epsilon)$ is at most $\epsilon$.

Proof: Consider an annulus $A=A_{\mathrm{sp}}(p, r, \epsilon)$, and suppose that there are four disjoint paths $\alpha_{0}, \alpha_{1}, \alpha_{2}, \alpha_{3}$ in $\widehat{T}_{\delta}$ that cross it. Let $B_{1}$ be the component of $\mathbb{S}^{2}-A$ inside the inner boundary component of $A$, and let $B_{2}$ be the outside component. Without loss of generality, we suppose that $\alpha_{0} \cup \alpha_{2}$ separate $\alpha_{1}$ from $\alpha_{3}$ inside $A$, that is, the circular order of these paths around $A$ agrees with the order of the indices.

Assume first that there are no paths in $\hat{\mathrm{T}}_{\delta} \cap A$ that join two of the paths $\alpha_{j}$, $j=0,1,2,3$. Then there must be paths in the dual tree $\beta_{0}, \beta_{1}, \beta_{2}, \beta_{3}$, such that $\beta_{j}$ is between $\alpha_{j-1}$ and $\alpha_{j}$ (indices mod 4), for each $j=0,1,2,3$. If $\alpha_{0}, \alpha_{1}, \alpha_{2}$ and $\alpha_{3}$ can all be connected to each other by paths in $A \cup B_{1}$, it follows that there are four crossings of the annulus $A_{\mathrm{sp}}(p, r, \epsilon / 3)$ in the $\epsilon / 3$ trunk of $\hat{\mathrm{T}}_{\delta}$, and we know that has small probability to happen anywhere, if $r$ is small, by Corollary 10.6. If neither of the paths $\alpha_{j}$ connects to another in $A \cup B_{1}$, the same argument applies to the dual tree, because the paths $\beta_{j}$ must all connect inside $A \cup B_{1}$. However, if two of the paths $\alpha_{j}$ connect in $A \cup B_{1}$, and one of the others does not connect to them, then also two of the paths $\beta_{j}$ connect. This implies that the $\epsilon / 3$ trunk gets within distance of $r$ from the dual trunk, and again this can be discarded as having small likelihood.

We are left to deal with the situation where there is a simple path $\gamma$ in $A \cap \widehat{T}_{\delta}$ that connects two of the paths $\alpha_{j}$. Note that for each pair of paths $\alpha_{j}$ there can be at most one such $\gamma$ connecting them. Also note that any path connecting $\alpha_{j}$ and $\alpha_{j+2}$ (indices mod 4) must cross either $\alpha_{j+1}$ or $\alpha_{j+3}$. Consequently, if we consider any four concentric annuli $A_{j}:=A_{\mathrm{sp}}\left(p, r_{j}, r_{j+1}\right), j=0,1,2,3,4$, with $r_{j}<r_{j+1}$ for each $j=0,1,2,3,4, r_{0}=r$, and $r_{5}=\epsilon$, at least one of them will have the property that inside it there is no path joining any two paths among the $\alpha_{j}$ 's. This allows a reduction to the previous case, and completes the proof.

Theorem 10.12: A.s., every simple path $\phi:[0,1) \rightarrow$ trunk has a $\operatorname{limit}_{t \rightarrow 1} \phi(t)$ in $\mathbb{S}^{2}$, and for every point $z \in \mathbb{S}^{2}$ there is a simple path $\phi:[0,1) \rightarrow$ trunk such that $\lim _{t \rightarrow 1} \phi(t)=z$.

Proof: Suppose that there are two distinct accumulation points, $x$ and $y$, of $\phi(t)$ as $t \rightarrow 1$, and let $m \in \mathbb{N}_{+}$satisfy $d_{s p}(x, y)>9 / m$. Then for each $t \in(0,1)$ 
the (spherical) diameter of $\phi([t, 1))$ is greater than $9 / m$. Let $a \in(0,1)$ be such that the diameter of $\phi([0, a])$ is at least $5 / m$. It easily follows that $\phi([a, 1)) \subset$ trunk $_{0}(1 / m)$. But since trunk $k_{0}(1 / m)$ is a compact finite tree (Corollary 10.3), and the restriction of $\phi$ to $[a, 1)$ is in trunk $k_{0}(1 / m)$, it follows that $\lim _{t \rightarrow 1} \phi(t)$ exists. Contradiction.

Let $z \in \mathbb{S}^{2}$ and $z^{\prime} \in \operatorname{trunk}_{0}(1), z^{\prime} \neq z$. We want to produce a simple path $\gamma \subset$ trunk starting at $z^{\prime}$ and tending to $z$. If $z \in$ trunk, then $z \in$ trunk $_{0}(1 / n)$ for some $n \in \mathbb{N}_{+}$, and the existence of $\gamma$ is clear. So suppose that $z \notin$ trunk. For each $n \in \mathbb{N}_{+}$, there is a point $z_{n} \in$ trunk $_{0}(1 / n)$ which is within distance $2 / n$ from $z$. Let $\beta_{n}$ be the arc from $z^{\prime}$ to $z_{n}$ in trunk $k_{0}(1 / n)$. For each $n$ and $m$, the intersection $\gamma_{n} \cap$ trunk $_{0}(1 / m)$ is a simple path. Since $\operatorname{trunk}_{0}(1 / m)$ is a compact finite topological tree, there is a subsequence $\gamma_{n_{j}}$ such that for each $m$ the Hausdorff $\operatorname{limit}_{\lim _{j}}\left(\gamma_{n_{j}} \cap\right.$ trunk $\left._{0}(1 / m)\right)$ exists, and is a simple path. Because $\gamma_{n}-B(z, 3 / m) \subset \operatorname{trunk}_{0}(1 / m)$ when $n \geqslant m$, it now follows that $\gamma:=\lim _{j} \gamma_{n_{j}}$ is a simple path. Moreover, it is clear that $z \in \gamma$ and $\gamma-\{z\} \subset$ trunk.

Proof of Theorem 1.6: We first prove that trunk is a topological tree. Clearly, the trunk is arcwise connected, since trunk $:=\bigcup_{n}$ trunk $_{0}(1 / n)$, and each trunk ${ }_{0}(1 / n)$ is arcwise connected. It is also clear that the trunk is dense in $\mathbb{S}^{2}$. Let $x, y \in$ trunk. Then there is some $n \in \mathbb{N}$ such that $x, y \in$ trunk $_{0}(1 / n)$, and there is a unique arc $\gamma_{1}$ joining $x$ and $y$ in trunk $k_{0}(1 / n)$. Let $\gamma_{2}$ be an arc joining $x$ and $y$ in trunk. Since the dual trunk is dense, it must intersect all connected components of $\mathbb{S}^{2}-\left(\gamma_{1} \cup \gamma_{2}\right)$. Since the dual trunk is disjoint from the trunk, it does not intersect $\gamma_{1} \cup \gamma_{2}$. Because the dual trunk is connected, it now follows that $\mathbb{S}^{2}-\left(\gamma_{1} \cup \gamma_{2}\right)$ is connected. Consequently, $\gamma_{1}=\gamma_{2}$, and the trunk is uniquely arcwise connected.

Let $n \in \mathbb{N}$ and let $t$ be the spherical distance between $\operatorname{trunk}_{0}(1 / n)$ and $\operatorname{trunk}_{0}^{\dagger}(1 / n)$. Since these are compact and disjoint, $t>0$. If $x \in$ trunk $_{0}(1 / n)$ and there is a $y \in$ trunk such that there is no path in trunk $\cap B(x, 3 / n)$ joining $x$ and $y$, then there must be a path in $\operatorname{trunk}_{0}^{\dagger}(1 / n)$ separating $x$ and $y$ in $B(x, 1 / n)$. (Indeed, if $\gamma$ is the path joining $x$ and $y$ and $p \in \gamma-B(x, 3 / n)$, then the path $\beta \subset$ trunk $^{\dagger}$ connecting two points $p^{\prime}$ and $p^{\prime \prime}$ that are near $p$ and are separated from each other by $\gamma$ near $p$, will have a subarc in $\operatorname{trunk}_{0}^{\dagger}(1 / n)$ separating $x$ and $y$ in $B(x, 1 / n)$.) Consequently, for every point $x \in$ trunk $_{0}(1 / n)$ and every $y \in$ trunk $\cap B(x, t) \cap B(x, 1 / n)$ there is a path in trunk $\cap B(x, 3 / n)$ joining $x$ and $y$. Hence, the union of all arcs that contain $x$ and are contained in trunk $\cap B(x, 3 / n)$ is an arcwise connected subset of trunk $\cap B(x, 3 / n)$ which contains trunk $\cap B(x, t) \cap B(x, 1 / n)$. This implies that trunk is locally arcwise connected, and so it is a topological tree. It is obviously dense in $\mathbb{S}^{2}$, and the 
proof of part (iii) is complete.

It is clear that for every $a, b \in \mathbb{S}^{2}$ there is some $\omega$ such that $(a, b, \omega) \in \mathfrak{T}$.

Let $\mathfrak{T}(\epsilon)$ be a subsequential scaling limit of $\mathfrak{T}_{\delta}(\epsilon)$. We prove that a.s. every $\omega$ such that $(a, b, \omega) \in \mathfrak{T}(\epsilon)$ for some $a, b \in \mathbb{S}^{2}$ is a simple path. Let $\epsilon_{1}>0$. It suffices to prove that the above statement holds with probability at least $1-\epsilon_{1}$. Let $V_{1} \subset \mathbb{S}^{2}$ be a finite set of points, and for each $\delta>0$ let $V_{1}^{\delta}$ be a collection of vertices of $\delta \mathbb{Z}^{2}$, each close to one point of $V_{1}$, and with $\left|V_{1}\right|=\left|V_{1}^{\delta}\right|$. By Theorem $10.2, V_{1}$ may be chosen so that with probability at least $1-\epsilon_{1}$ the subtree of $\hat{\mathrm{T}}_{\delta}$ spanned by $V_{1}^{\delta}$ contains $\operatorname{trunk}_{\delta}(\epsilon)$, for all sufficiently small $\delta$. This implies that each $\omega_{a, b}(\epsilon)$ is a subarc of $\omega_{v, u}$ for some $v, u \in V_{1}^{\delta}$. Because for every pair of points $v, u \in V_{1}$ the scaling limit of the LERW from $v$ to $u$ is a simple path, it follows that with probability at least $1-\epsilon_{1}$ for each $(a, b, \omega) \in \mathfrak{T}(\epsilon), \omega$ is a simple path.

We may now conclude that a.s. for every $(a, b, \omega) \in \mathfrak{T}$, the set

$$
\omega-(\bar{B}(a, \epsilon) \cup \bar{B}(b, \epsilon))
$$

is a 1 -manifold, that is, a disjoint union of simple paths. Therefore, $\omega^{\prime}:=$ $\omega-\{a, b\}$ is a 1 -manifold. This means that each component of $\omega^{\prime}$ is an arc with endpoints in $\{a, b\}$. It is clear that $\omega$ may be oriented as a path from $a$ to $b$. Suppose that $\omega$ visits $a$ more than once. If $a \neq b$, it then follows that $a \in$ trunk, and there is a simple closed path in trunk containing $a$. This is impossible, since trunk is a topological tree. Hence $a$, and similarly $b$, are each visited only once in $\omega$, which implies that $\omega$ is a simple path if $a \neq b$. If $a=b$, the only possibility is that $\omega=\{a\}$ or that $\omega$ is a simple closed path. This proves the first and second statements in (ii).

Observe that if there is simple curve $\alpha \subset$ trunk such that $\bar{\alpha}=\alpha \cup\{a\}$, then $a$ must be in the dual trunk, for the dual trunk is connected, intersects both components of $\mathbb{S}^{2}-\bar{\alpha}$, and is disjoint from trunk. This is a rare event, by Remark 3.2 (or Corollary 10.4). This proves (ii).

Corollary 10.11 proves (iv).

The first claim in (i) is obvious. Suppose that $a \neq b$ are such that there are two sets $\omega$ and $\omega^{\prime}$ with $(a, b, \omega),\left(a, b, \omega^{\prime}\right) \in \mathfrak{T}$. We know that $\omega$ and $\omega^{\prime}$ are simple paths. If $\omega \neq \omega^{\prime}$, then there is a simple closed path, say $\gamma$, contained in $\bar{\omega} \cup \bar{\omega}^{\prime}$. But as above, $\gamma$ must intersect the dual trunk, since the dual trunk is connected and dense. This implies that $a$ or $b$ are in the dual trunk. This completes the proof of (i), and of the theorem. 
Remark 10.13: Uniqueness of paths. We have seen in the above proof that the path in trunk from $a$ to $b$ is unique when $\{a, b\} \cap$ trunk $^{\dagger}=\emptyset$. The converse is also easily established.

Remark 10.14 (Reconstructing trunk ${ }^{\dagger}$ ): It can be shown that the scaling limit dual trunk can be reconstructed from the trunk. This can be seen from Remark 10.13. Another description of the dual trunk from the trunk is as follows. Given distinct $x, y \in \mathbb{S}^{2}$ - trunk, let $\gamma(x, y)$ be the (unique) arc in $\mathbb{S}^{2}-$ trunk with endpoints $x, y$. Then

$$
\text { trunk }^{\dagger}=\bigcup\left\{\gamma(x, y)-\{x, y\}: x \neq y, x, y \in \mathbb{S}^{2}-\text { trunk }\right\} .
$$

To prove this, it suffices to establish that $\gamma(x, y)$ is unique, which follows from the fact that trunk is connected and dense.

Remark 10.15: Consider the metric $d^{*}$ on trunk, where $d^{*}(x, y)$ is the spherical diameter of the unique (possibly degenerate) arc joining $x$ and $y$ in trunk. Since trunk is locally arcwise connected, this new metric on trunk is compatible with the topology of trunk as a subset of $\mathbb{S}^{2}$. Let trunk $\mathbf{k}_{*}$ denote the completion of this metric. Then trunk $k_{*}$ is a compact topological tree, and is naturally homeomorphic with the ends compactification of trunk. Since $d^{*}$ majorizes the spherical metric, there is a natural projection $\pi$ : trunk $\mathbf{k}_{*} \rightarrow \mathbb{S}^{2}$, whose restriction to trunk is the identity. It is easy to see that every point $p \in \mathbb{S}^{2}-$ trunk $^{\dagger}$ has a unique preimage under $\pi$, and for points $p \in$ trunk $^{\dagger}$, the degree of $p$ in trunk $k^{\dagger}$ is equal to $\left|\pi^{-1}(p)\right|$.

Consider some $o \in \mathbb{S}^{2}$, and let $\mathfrak{T}^{o}$ be the appropriate "slice" of $\mathfrak{T}$, that is, $\mathfrak{T}^{o}:=\{(b, \omega):(o, b, \omega) \in \mathfrak{T}\}$. One can show that if $o \notin$ trunk $^{\dagger}$, then $\mathfrak{T}^{o}$ is homeomorphic with trunk $k_{*}$, and $\pi: \mathfrak{T}^{\circ} \rightarrow \mathbb{S}^{2}$ is the projection onto the first

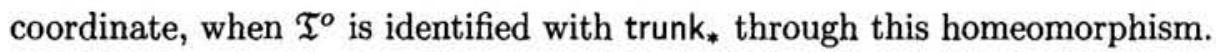

\section{Free and wired trunks and conformal invariance}

We now want to give a precise formulation to a conformal invariance conjecture for the UST scaling limit, and prove that it follows from the conjectured conformal invariance of the LERW scaling limit. (Such conformal invariance conjectures seem to be floating in the air these days, with roots in the physics community.) The conformal automorphisms of $\mathbb{S}^{2}$ are Möbius transformations. We conjecture that the different notions of scaling limits of UST in $\mathbb{S}^{2}$, which were introduced in the previous section, exist (without a need to pass to a subsequence) and are invariant under Möbius transformations. Moreover, the scaling limits in subdomains $D \subset \mathbb{S}^{2}$ should be invariant under conformal homeomorphisms $f: D \rightarrow D^{\prime} \subset \mathbb{S}^{2}$. This is a significantly stronger statement, since the 
Möbius transformations of $\mathbb{S}^{2}$ form a 6-dimensional group, while the space of conformal homeomorphisms from the unit disk onto subdomains of $\mathbb{S}^{2}$ is infinite dimensional.

To formulate more precisely the invariance under conformal homeomorphisms of subdomains $f: D \rightarrow D^{\prime}$, we need first to discuss UST scaling limits in subdomains of $\mathbb{S}^{2}$. This will be now explained.

For simplicity, we restrict our attention to simply connected domains $D \varsubsetneqq \mathbb{R}^{2}$ whose boundary $\partial D$ is a simple closed path. Let $p_{0} \in D$ be some basepoint. Let $\mathrm{FT}_{\delta}^{D}$ be the uniform spanning tree of $G(D, \delta)$, with free boundary conditions, and let WT ${ }_{\delta}^{D}$ be the uniform spanning tree of $G(D, \delta)$ with wired boundary conditions. Let $\mathbb{S}_{D}^{2}$ be the metric space obtained from $\mathbb{S}^{2}$ by contracting $\mathbb{S}^{2}-D$ to a single point. Then we may think of $\mathrm{WT}_{\delta}^{D}$ as a random point in $\mathcal{H}\left(\mathbb{S}_{D}^{2}\right)$, which is a.s. a tree. The tree $\mathrm{FT}_{\delta}^{D}$ may be thought of as a random point in $\mathcal{H}(\bar{D})$, which is a.s. a tree.

Let $\mathfrak{W T} \mathfrak{T}_{\delta}^{D}:=\mathfrak{T}\left(\mathrm{WT}_{\delta}^{D}\right)$ and $\mathfrak{F T} \mathfrak{T}_{\delta}^{D}:=\mathfrak{T}\left(\mathrm{FT}_{\delta}^{D}\right)$, that is, the wired paths ensemble $\mathfrak{W} \mathfrak{T}_{\delta}$ is defined from the wired tree $\mathrm{WT}_{\delta}$ in exactly the same way that the ordinary paths ensemble $\mathfrak{T}_{\delta}$ was defined from $\widehat{T}_{\delta}$, and similarly for $\mathfrak{F T}_{\delta}$. Note that $\mathfrak{W T}_{\delta} \in \mathcal{H}\left(\mathbb{S}_{D}^{2} \times \mathbb{S}_{D}^{2} \times \mathcal{H}\left(\mathbb{S}_{D}^{2}\right)\right)$ and $\mathfrak{F T}_{\delta} \in \mathcal{H}(\bar{D} \times \bar{D} \times \mathcal{H}(\bar{D}))$. Also the definitions of the scaling limits and the trunk are the same as in the previous section.

Theorem 11.1: Let $D \subset \mathbb{R}^{2}$ be a domain whose boundary is a $C^{1}$-smooth simple closed curve.

(i) Theorem 10.2, with $\mathbb{S}^{2}$ replaced by $D$, holds for the free and wired spanning trees in $D$.

(ii) The free scaling limit trunk in $D$ is disjoint from $\partial D$, in every (subsequential) scaling limit.

(iii) The free scaling limit trunk in $D$ is disjoint from the scaling limit trunk of the dual tree (which is wired), in every (subsequential) scaling limit.

There are simply connected domains where $\partial D$ is not a simple closed curve and (ii) fails: the domain $(0,2) \times(0,2)-\bigcup_{n=1}^{\infty}(0,1] \times\{1 / n\}$ is an example.

LEMMA 11.2: There is an absolute constant $C>0$ such that the following holds true. Let $D$ be as in Theorem 11.1. Then there is a $\delta_{0}=\delta_{0}(D)>0$ with the following property. Suppose that $\delta$ and $\delta_{1}$ are numbers satisfying $0<\delta \leqslant \delta_{1} \leqslant \delta_{0}$ and $A$ is a connected subgraph of $G(D, \delta)$ with diameter at least $\delta_{1}$. Further suppose that $p \in \mathrm{V}(G(D, \delta))$ has distance $\delta_{1}$ to $A$. Then the probability that a random walk on $G(D, \delta)$ starting at $p$ will get to distance $C \delta_{1}$ from $p$ before hitting $A$ is less than $1 / 2$. 
Proof: The proof is similar to the proof of Lemma 10.9. Let $Z=Z(t)$ be the set of vertices with distance at least $t$ from $p$, where we take $t>4 \delta_{1}$. Let $A^{\prime}$ be a component of $A \cap B\left(p, 3 \delta_{1}\right)$ containing some point at distance $\delta_{1}$ to $p$ and having diameter at least $\delta_{1}$. For $v \in \mathrm{V}(G(D, \delta))$, let $h(v)$ be the probability that a random walk starting from $v$ will reach $Z$ before hitting $A^{\prime}$. Then $h$ is discreteharmonic, and minimizes Dirichlet energy among functions that are 1 on $Z$ and 0 on $A^{\prime}$. As in the proof of Lemma 10.9, it follows that the Dirichlet energy of $h$ is at most $O(1) / \log \left(t / \delta_{1}\right)$. Let $B$ be the set of vertices $v$ at distance at most $2 \delta_{1}$ from $p$ such that $h(v) \geqslant h(p)$, and let $B^{\prime}$ be the component of $B$ containing $p$. Note that the diameter of $B^{\prime}$ is at least $\delta_{1}$, as $B^{\prime}$ must neighbor with some vertex with distance $2 \delta_{1}$ from $p$, by the maximum principle for $h$. As in the proof of Lemma 10.9 , it can be shown that when $\delta_{1}$ is sufficiently small (how small depends on the scale in which $\partial D$ appears smooth), one can find $O(1) / \delta$ disjoint paths in $\delta \mathbb{Z}^{2} \cap D$ connecting $A^{\prime}$ to $B^{\prime}$, each of combinatorial length $O(1) / \delta$. Because $h$ is zero on $A^{\prime}$ and at least $h(p)$ on $B^{\prime}$, it follows that the Dirichlet energy of $h$ is at least $O(1) h(p)$. We conclude that $h(p) \leqslant O(1) / \log \left(t / \delta_{1}\right)$, which proves the lemma.

Proof of Theorem 11.1: The proof for (i) in the wired case is the same as the proof of Theorem 10.2 (and we don't need to assume anything about $D$ ). The free case is the same, except that one needs to appeal to Lemma 11.2. Assuming (ii), the proof of (iii) is identical to the proof of Theorem 10.7. The proof of (ii) is also the same as the proof of 10.7 , except that one needs to find the appropriate substitutes for Lemma 10.8 and Corollary 10.6; namely, for every $\epsilon>0$ there is an $\epsilon_{0}>0$ such that for all $\delta \in\left(0, \epsilon_{0}\right)$ with probability at least $1-\epsilon$ all points of degree three in the $\epsilon$-trunk of $\mathrm{FT}_{\delta}^{D}$ have distance at least $\epsilon_{0}$ from $\partial D$, and the probability that there is a point $p \in \partial D$ such that there are two disjoint crossings of the annulus $A\left(p, \epsilon_{0}, \epsilon\right)$ in the $\epsilon$-trunk of $\mathrm{WT}_{\delta}^{D}$ is at most $\epsilon$. The latter statement follows from the proof of Corollary 10.6.

It remains to prove the appropriate substitute for 10.8. Consider three distinct points in $D, p_{1}, p_{2}, p_{3}$, and for $\delta>0$ let $p_{1}^{\prime}, p_{2}^{\prime}, p_{3}^{\prime}$ be a triple of points in $\delta \mathbb{Z}^{2}$ which is close to $p_{1}, p_{2}, p_{3}$, respectively. Let $m$ be the meeting point of $p_{1}^{\prime}, p_{2}^{\prime}, p_{3}^{\prime}$ in $\mathrm{FT}_{\delta}^{D}$, and let $B$ be any disk whose center is in $\partial D$ and which does not intersect $\left\{p_{1}^{\prime}, p_{2}^{\prime}, p_{3}^{\prime}\right\}$. Let $B^{\prime}$ and $B^{\prime \prime}$ be disks concentric with $B$ of $1 / 2$ and $1 / 4$ of its size, respectively. By part (i), it suffices to prove that with probability going to 1 as $\epsilon_{0} \rightarrow 0$ and $\delta \rightarrow 0, m$ is not within distance $\epsilon_{0}$ from $\partial D \cap B^{\prime \prime}$.

Suppose that $m \in B$. Let $\gamma_{1}, \gamma_{2}, \gamma_{3}$ be the $\operatorname{arcs}$ of $\mathrm{FT}_{\delta}^{D}$ that join $m$ to $p_{1}^{\prime}, p_{2}^{\prime}, p_{3}^{\prime}$, respectively, and let $\gamma_{j}^{\prime}$ be the largest initial segment of $\gamma_{j}$ that is contained in $B$, 
$j=1,2,3$. There is a unique $k \in\{1,2,3\}$ such that $\bigcup_{j \neq k} \gamma_{j}^{\prime}$ separates $\gamma_{k}^{\prime}$ from $\partial D$ in $B$. By symmetry, it suffices to estimate the probability that $m$ is close to $B \cap \partial D$ and $k=3$. We generate $m$ in the following way. Let $\gamma$ be a LERW from $p_{1}^{\prime}$ to $p_{2}^{\prime}$ in $\delta \mathbb{Z}^{2} \cap D$. Let $X$ be a random walk on $\delta \mathbb{Z}^{2} \cap D$ starting at $p_{3}^{\prime}$, let $\tau_{\gamma}$ be the first time $t$ where $X(t) \in \gamma$, and let $\tau_{1}$ be the first time $t$ when $X(t)$ is incident with an edge intersecting $\partial D \cap B^{\prime}$. Then we may take $m=X\left(\tau_{\gamma}\right)$. Consider the event $\mathcal{A}$ where $m \in B, k=3$ and $\tau_{1} \geqslant \tau_{\gamma}$. With high probability, the points $X(t), t \leqslant \tau_{1}$, which are close to $B^{\prime} \cap \partial D$, are also close to $X\left(\tau_{1}\right)$. This is just a property of simple random walk absorbed at $B^{\prime} \cap \partial D$. Consequently, on $\mathcal{A}$, with high probability, if $m$ is close to $B \cap \partial D$, then $\gamma$ passes close to $X\left(\tau_{1}\right)$. Since the probability that $\gamma$ passes near any point, which is not too close to $p_{1}^{\prime}$ and $p_{2}^{\prime}$, is small (Remark 3.2 applies here), and $X\left(\tau_{1}\right)$ is independent of $\gamma$, we see that $\mathcal{A}$ has arbitrarily small probability.

We now need to consider the case $k=3$ and $\tau_{1}<\tau_{\gamma}$. Let $\tau_{1}^{\prime}$ be the first $t \geqslant \tau_{1}$ such that $X(t) \notin B$, and let $\tau_{2}$ be the first $t \geqslant \tau_{1}^{\prime}$ such that $X(t)$ is incident with an edge intersecting $\partial D \cap B^{\prime}$. Inductively, let $\tau_{n}^{\prime}$ be the first $t \geqslant \tau_{n}$ such that $X(t) \notin B$ and let $\tau_{n+1}$ be the first $t \geqslant \tau_{n}^{\prime}$ such that $X(t)$ is incident with an edge intersecting $\partial D \cap B^{\prime}$. Note that if $k=3$ and $\tau_{\gamma}>\tau_{1}$, then $\tau_{\gamma}>\tau_{1}^{\prime}$, for the random walk $X$ must go around $\gamma_{1}^{\prime} \cup \gamma_{2}^{\prime}$ before hitting $\gamma$. Similarly, if $k=3$ and $\tau_{\gamma}>\tau_{1}$, then $\tau_{\gamma} \in\left(\tau_{n}^{\prime}, \tau_{n+1}\right)$ for some $n \in \mathbb{N}$. If we fix a finite $k \in \mathbb{N}$, then the same argument as above shows that with high probability, $\gamma$ does not pass close to the set $\left\{X\left(\tau_{n}\right): n=1,2, \ldots, k\right\}$. Because $\mathbf{P}\left[\tau_{k}^{\prime}<\gamma\right] \rightarrow 0$ as $k \rightarrow \infty$, uniformly in $\delta$, the required result follows.

Suppose that $D$ and $D^{\prime}$ are two domains in $\mathbb{R}^{2}$ such that the boundaries $\partial D, \partial D^{\prime}$ are simple closed paths in $\mathbb{R}^{2}$. Then there is a conformal homeomorphism $f: D \rightarrow D^{\prime}$. Moreover, $f$ extends continuously to a homeomorphism of $\bar{D}$ onto $\bar{D}^{\prime}$, which we will also denote by $f$. It follows that $f$ induces maps

$$
\begin{gathered}
f_{W}: \mathcal{H}\left(\mathbb{S}_{D}^{2} \times \mathbb{S}_{D}^{2} \times \mathcal{H}\left(\mathbb{S}_{D}^{2}\right)\right) \rightarrow \mathcal{H}\left(\mathbb{S}_{D^{\prime}}^{2} \times \mathbb{S}_{D^{\prime}}^{2} \times \mathcal{H}\left(\mathbb{S}_{D^{\prime}}^{2}\right)\right), \\
f_{F}: \mathcal{H}(\bar{D} \times \bar{D} \times \mathcal{H}(\bar{D})) \rightarrow \mathcal{H}(\bar{D} \times \bar{D} \times \mathcal{H}(\bar{D})) .
\end{gathered}
$$

Theorem 11.3: Let $D \subset \mathbb{R}^{2}$ be a domain whose boundary is a $C^{1}$-smooth simple closed path. Assuming Conjecture 1.2, the following is true.

(i) The free and the wired UST scaling limits, $\mathfrak{F T}, \mathfrak{W T}$, in $D$ exist. (That is, they do not depend on the sequence of $\delta$ tending to 0 .)

(ii) If $f: D \rightarrow D^{\prime}$ is a conformal homeomorphism between such domains, then $f_{W}$ is measure preserving from the law of $\mathfrak{W T}$ in $D$ to the law of $\mathfrak{W T}$ in $D^{\prime}$, and similarly for free boundary conditions. 
Proof: The proof for wired boundary conditions follows from Wilson's algorithm and Theorem 11.1. The easy details are left to the reader.

For the free boundary conditions, observe that $\mathrm{WT}_{\delta}^{D}$ is dual to $\mathrm{FT}_{\delta}^{D}$ (on the dual grid). Remark 10.14 is also valid in the present setting, and shows that the free scaling limit trunk can be reconstructed from the wired scaling limit trunk. It is easy to see that the free scaling limit $\mathfrak{F T}$ can be reconstructed from the trunk. Hence, conformal invariance of the wired UST implies conformal invariance of the free.

\section{Speculations about the Peano curve scaling limit}

This section will discuss the Peano curve winding between the UST and its dual. From here on, the discussion will be somewhat speculative, and we omit proofs, not because the proofs are particularly hard, but because the paper is long enough as it is, and it is not clear when another paper on this subject will be produced.

This Peano curve was briefly mentioned in [BLPS98]. Consider the set of points $\theta_{\delta} \subset \mathbb{R}^{2}$ which have the same Euclidean distance from $\hat{\mathrm{T}}_{\delta}$ as from its dual $\hat{\mathrm{T}}_{\delta}^{\dagger}$. It is easy to verify that $\theta_{\delta}$ is a simple path in a square grid $G_{P}(\delta)$, of mesh $\delta / 2$, which visits all the vertices in that grid. Set $\widehat{\theta}_{\delta}:=\theta_{\delta} \cup\{\infty\}$. Then $\widehat{\theta}_{\delta}$ is a.s. a simple closed path in $\mathbb{S}^{2}$ passing through $\infty$.

To consider the scaling limit of $\widehat{\theta}_{\delta}$, it is no use to think of it as a set of points in $\mathbb{S}^{2}$, because then the scaling limit will be all of $\mathbb{S}^{2}$. Rather, one needs to parameterize $\widehat{\theta}_{\delta}$ in some way. One natural parameterization would be by the area of its $\delta / 2$-neighborhood, but there are several other plausible parameterizations. Another, more sophisticated approach, would be to think of $\widehat{\theta}_{\delta}$ as defining a circular order on the set $\hat{\theta}_{\delta}$. The circular order $R_{\delta}$ is a closed subset of $\left(\mathbb{S}^{2}\right)^{4}$, and $(a, b, c, d) \in R_{\delta}$ iff $a, b, c, d \in \widehat{\theta}_{\delta}$ and $\{a, c\}$ separates $b$ from $d$ on $\widehat{\theta}_{\delta}$. Then the (subsequential) scaling limit of $\hat{\theta}_{\delta}$ may be taken as the weak limit of the law of $R_{\delta}$ in $\mathcal{H}\left(\left(\mathbb{S}^{2}\right)^{4}\right)$.

Let $\theta$ denote the Peano curve scaling limit, defined as a path, or as a circular order, or some other reasonable definition. Here is what we believe to be a description of $\theta$, in terms of the scaling limit of the UST. Recall that in Remark 10.15 , we have introduced a completion trunk $k_{*}$ of the trunk, in the metric $d^{*}$, where the distance between any two points of trunk is the diameter of the arc connecting them, and that $\pi$ : trunk $\boldsymbol{k}_{*} \rightarrow \mathbb{S}^{2}$ is the natural projection. Consider the joint distribution of trunk $k_{*}$ and the dual trunk ${ }_{*}^{\dagger}$, and let $\pi^{\dagger}$ denote the projection $\pi^{\dagger}$ : trunk $k_{*}^{\dagger} \rightarrow \mathbb{S}^{2}$. Let $\tilde{\theta}$ be the set of points $(p, q) \in$ trunk $_{*} \times$ trunk $_{*}^{\dagger}$ such that $\pi p=\pi^{\dagger} q$. Then $\tilde{\theta}$ is a simple closed path, and the map $\tilde{\theta} \mapsto \mathbb{S}^{2}$ defined by 
$(p, q) \mapsto \pi p$ gives the scaling limit $\theta$.

Fix some $a, b \in \mathbb{R}^{2}, a \neq b$. Let $\omega_{a, b}$ be the path such that $\left(a, b, \omega_{a, b}\right) \in \mathfrak{T}$ (this $\omega_{a, b}$ is a.s. unique), and let $\omega_{a, b}^{\dagger}$ be such that $\left(a, b, \omega_{a, b}^{\dagger}\right) \in \mathfrak{T}^{\dagger}$. Then $\omega_{a, b}$ and $\omega_{a, b}^{\dagger}$ are a.s. simple paths. Let $D_{1}$ and $D_{2}$ be the two components of $\mathbb{S}^{2}-\left(\omega_{a, b} \cup \omega_{a, b}^{\dagger}\right)$. A.s. $\infty \in D_{1} \cup D_{2}$, and without loss of generality take $\infty \in D_{1}$. It is then clear that the part of $\theta$ which is between $a, b$ and does not contain $\infty$ is $\bar{D}_{2}$, and that the part which does contain $\infty$ is $\bar{D}_{1}$. Suppose that we condition on $\omega_{a, b}$ and on $\omega_{a, b}^{\dagger}$, and look at some point $c \in D_{2}$. We'd like to know the distribution of the part of $\theta$ between $c$ and $a$ which does not include $b$, say. Recall that on a finite planar graph, we may generate the UST and the dual UST by a modification of Wilson's algorithm, where at each step in which we start from a vertex in the graph, the dual tree built up to that point acts as a free boundary component, and the tree built up to that point acts as an absorbing wired boundary component, and at steps in which we start from a dual vertex, the tree built up to that point acts as a free boundary component, and the dual tree built up to that point acts as an absorbing wired boundary component. Consequently, we let $\alpha$ be the scaling limit of LERW on $\delta \mathbb{Z}^{2}-\omega_{a, b}^{\dagger}$ starting at $c$ that stops when it hits $\omega_{a, b}$. Then we let $\alpha^{\dagger}$ be the scaling limit of LERW on $\delta \mathbb{Z}^{2}-\left(\alpha \cup \omega_{a, b}\right)$ starting at $c$ that stops when it hits $\omega^{\dagger} a, b$. (Since $c \in \alpha$, to define this requires taking a limit as the starting point tends to $c$.) Then $\alpha \cup \alpha^{\dagger}$ separates $D_{2}$ into two regions, say $D_{2}^{\prime}$ and $D_{2}^{\prime \prime}$, and if $b \notin \bar{D}_{2}^{\prime}$, then $D_{2}^{\prime}$ is the part of $\theta$ "separated" from $b$ by $\{a, c\}$.

In the above construction, the domain $D_{2}$ was considered with mixed boundary conditions. One arc of $\partial D_{2}-\{a, b\}$ was taken as wired, while the other was free. The resulting Peano path scaling limit $\theta$ is a path joining $a$ and $b$ in $\bar{D}_{2}$. From Conjecture 1.2 should follow a conformal invariance result for UST in such domains with mixed boundary conditions. Therefore, having an understanding of the law of the Peano curve for one triplet $(D, a, b)$, where $a$ and $b$ are distinct points in $\partial D$, which is a simple closed curve, suffices for any other such triplet. This suggests that we should take the simplest possible such configuration, that is, $D=\mathbb{H}$, the upper half plane, $a=0, b=\infty$. Suppose that we then take a point $c \in \mathbb{H}$ and condition on the part of $\theta$ between 0 and $c$ and separated from $\infty$. The effect of that on $\theta$ in the remaining subdomain $D_{c}$ of $\mathbb{H}$ is all in the boundary $\partial D_{c}$. This is a kind of Markovian property for the Peano curve, similar to the property given by Lemma 4.3. By taking the conformal map from $D_{c}$ to $\mathbb{H}$, which fixes $\infty$, takes $c$ to 0 , and is appropriately normalized at $\infty$, we may return to base one. This suggests that, as we have claimed in the introduction, a representation of the Peano curve scaling limit is similar to the SLE representation of the LERW 
from 0 to $\partial U$ which we have introduced. The analogue of the Löwner differential equation for this situation is (1.6). Due to the Markovian nature of the Peano curve, the corresponding parameter $\zeta$ in (1.6) should have the form $\zeta=\mathrm{B}(\kappa t)$, where $\mathrm{B}$ is Brownian motion on $\mathbb{R}$ starting at 0 , and $\kappa$ is some constant. One can, in fact, show that $\kappa=8$, by deriving an appropriate analogue of Cardy's [Car92] conjectured formula, using the representation (1.6) and the techniques of Section 9. The details will appear elsewhere.

\section{References}

[Aiz] M. Aizenman, Continuum limits for critical percolation and other stochastic geometric models, Preprint. http://xxx.lanl.gov/abs/math-ph/9806004.

[ABNW] M. Aizenman, A. Burchard, C. M. Newman and D. B. Wilson, Scaling limits for minimal and random spanning trees in two dimensions, Preprint. http://xxx.lanl.gov/abs/math/9809145.

[ADA] M. Aizenman, B. Duplantier and A. Aharony, Path crossing exponents and the external perimeter in $2 D$ percolation, Preprint. http://xxx.lanl.gov/abs/cond-mat/9901018.

[Ald90] D. J. Aldous, The random walk construction of uniform spanning trees and uniform labelled trees, SIAM Journal on Discrete Mathematics 3 (1990), 450-465.

[Ben] I. Benjamini, Large scale degrees and the number of spanning clusters for the uniform spanning tree, in Perplexing Probability Problems: Papers in Honor of Harry Kesten (M. Bramson and R. Durrett, eds.), Boston, Birkhäuser, to appear.

[BLPS98] I. Benjamini, R. Lyons, Y. Peres and O. Schramm, Uniform spanning forests, Preprint.

http://www.wisdom.weizmann.ac.il/〜schramm/papers/usf/.

[BJPP97] C. J. Bishop, P. W. Jones, R. Pemantle and Y. Peres, The dimension of the Brownian frontier is greater than 1, Journal of Functional Analysis 143 (1997), 309-336.

[Bow] B. H. Bowditch, Treelike structures arising from continua and convergence groups, Memoirs of the American Mathematical Society, to appear.

[Bro89] A. Broder, Generating random spanning trees, in 30th Annual Symposium on Foundations of Computer Science, IEEE, Research Triangle Park, NC, 1989, pp. 442-447. 
[BP93] R. Burton and R. Pemantle, Local characteristics, entropy and limit theorems for spanning trees and domino tilings via transfer-impedances, The Annals of Probability 21 (1993), 1329-1371.

[Car92] J. L. Cardy, Critical percolation in finite geometries, Journal of Physics A 25 (1992), L201-L206.

[DD88] B. Duplantier and F. David, Exact partition functions and correlation functions of multiple Hamiltonian walks on the Manhattan lattice, Journal of Statistical Physics 51 (1988), 327-434.

[Dur83] P. L. Duren, Univalent Functions, Springer-Verlag, New York, 1983.

[Dur84] R. Durrett, Brownian Motion and Martingales in Analysis, Wadsworth International Group, Belmont, California, 1984.

[Dur91] R. Durrett, Probability, Wadsworth \& Brooks/Cole Advanced Books \& Software, Pacific Grove, CA, 1991.

[EK86] S. N. Ethier and T. G. Kurtz, Markov Processes, Wiley, New York, 1986.

[Gri89] G. Grimmett, Percolation, Springer-Verlag, New York, 1989.

[Häg95] O. Häggström, Random-cluster measures and uniform spanning trees, Stochastic Processes and their Applications 59 (1995), 267-275.

[Itô61] K. Itô, Lectures on Stochastic Processes, Notes by K. M. Rao, Tata Institute of Fundamental Research, Bombay, 1961.

[Jan12] Janiszewski, Journal de l'Ecole Polytechnique 16 (1912), 76-170.

[Ken98a] R. Kenyon, Conformal invariance of domino tiling, Preprint. http://topo.math.u-psud.fr/ kenyon/confinv.ps.Z.

[Ken98b] R. Kenyon, The asymptotic determinant of the discrete laplacian, Preprint. http://topo.math.u-psud.fr/ “kenyon/asymp.ps.Z.

[Ken99] R. Kenyon, Long-range properties of spanning trees, Preprint.

[Ken] R. Kenyon, in preparation.

[Kes87] H. Kesten, Hitting probabilities of random walks on $\mathbb{Z}^{d}$, Stochastic Processes and their Applications 25 (1987), 165-184.

[Kuf47] P. P. Kufarev, A remark on integrals of Löwner's equation, Doklady Akademii Nauk SSSR (N.S.) 57 (1947), 655-656.

[LPSA94] R. Langlands, P. Pouliot and Y. Saint-Aubin, Conformal invariance in twodimensional percolation, Bulletin of the American Mathematical Society (N.S.) 30 (1994), 1-61.

[Law93] G. F. Lawler, A discrete analogue of a theorem of Makarov, Combinatorics, Probability and Computing 2 (1993), 181-199. 
[Law] G. F. Lawler, Loop-erased random walk, in Perplexing Probability Problems: Papers in Honor of Harry Kesten (M. Bramson and R. Durrett, eds.), Boston, Birkhäuser, to appear.

[Löw23] K. Löwner, Untersuchungen über schlichte konforme abbildungen des einheitskreises, I, Mathematische Annalen 89 (1923), 103-121.

[Lyo98] R. Lyons, $A$ bird's-eye view of uniform spanning trees and forests, in Microsurveys in Discrete Probability (Princeton, NJ, 1997), American Mathematical Society, Providence, RI, 1998, pp. 135-162.

[MR] D. E. Marshall and S. Rohde, in preparation.

[MMOT92] J. C. Mayer, L. K. Mohler, L. G. Oversteegen and E. D. Tymchatyn, Characterization of separable metric $\mathbb{R}$-trees, Proceedings of the American Mathematical Society 115 (1992), 257-264.

[MO90] J. C. Mayer and L. G. Oversteegen, A topological characterization of $\mathbb{R}$-trees, Transactions of the American Mathematical Society 320 (1990), 395-415.

[New92] M. H. A. Newman, Elements of the Topology of Plane Sets of Points, second edition, Dover, New York, 1992.

[Pem91] R. Pemantle, Choosing a spanning tree for the integer lattice uniformly, The Annals of Probability 19 (1991), 1559-1574.

[Pom66] C. Pommerenke, On the Loewner differential equation, The Michigan Mathematical Journal 13 (1966), 435-443.

[Rus78] L. Russo, A note on percolation, Zeitschrift für Wahrscheinlichkeitstheorie und Verwandte Gebiete 43 (1978), 39-48.

[SD87] H. Saleur and B. Duplantier, Exact determination of the percolation hull exponent in two dimensions, Physical Review Letters 58 (1987), 23252328 .

[Sch] O. Schramm, in preparation.

[Sla94] G. Slade, Self-avoiding walks, The Mathematical Intelligencer 16 (1994), 29-35.

[SW78] P. D. Seymour and D. J. A. Welsh, Percolation probabilities on the square lattice, in Advances in Graph Theory (Cambridge Combinatorial Conference, Trinity College, Cambridge, 1977), Annals of Discrete Mathematics 3 (1978), 227-245.

[TW98] B. Tóth and W. Werner, The true self-repelling motion, Probability Theory and Related Fields 111 (1998), 375-452.

[Wil96] D. B. Wilson, Generating random spanning trees more quickly than the cover time, in Proceedings of the Twenty-eighth Annual ACM Symposium on the Theory of Computing (Philadelphia, PA, 1996), ACM, New York, 1996 , pp. 296-303. 